\title{
Implementation of a stress management program in outpatients with type 2 diabetes mellitus: a randomized controlled trial
}

\author{
Efi Koloverou, ${ }^{1}$ Nikolaos Tentolouris, ${ }^{2}$ Chryssa Bakoula, ${ }^{3}$ \\ Christina Darviri, ${ }^{1}$ George Chrousos ${ }^{1,4}$
}

${ }^{1}$ Postgraduate Course Stress Management and Health Promotion, Athens University Medical School; ${ }^{2} 1^{\text {st }}$ Department of Propedeutic Medicine, Athens University Medical School, "Laiko" General Hospital; ${ }^{3}{ }^{\text {nd }}$ Department of Pediatrics, Athens University Medical School, "P. \& A. Kyriakou" Children's Hospital; ${ }^{4} 1^{\text {st }}$ Department of Pediatrics, Athens University Medical School, “Aghia Sophia” Children’s Hospital; Athens, Greece

\begin{abstract}
OBJECTIVE: Although there is scientific evidence that stress adversely affects metabolic control, only a few studies have examined the role of stress management in improving glycemic control in patients with type 2 diabetes mellitus (DM). In this study, we investigated the effect of a relaxation technique on levels of stress and glycemic control. DESIGN: A total of 53 patients with type $2 \mathrm{DM}$ were randomly assigned to undergo either an 8-week stress management program, consisting of $10 \mathrm{~min}$ of diaphragmatic breathing and $15 \mathrm{~min}$ of progressive muscle relaxation twice per day ( $n=25$, intervention group), or not $(n=28$, control group). Perceived stress, health locus of control and HbA1c were primary outcomes and were measured before and after intervention. RESULTS: In the intervention group, perceived stress score (PSS) and HbA1c had decreased significantly $(\mathrm{P}<0.05)$ by the end of the program. Specifically concerning the PSS, the higher the initial levels of perceived stress the greater the benefit of the intervention. No other significant changes were found. CONCLUSIONS: Our results show a beneficial role of stress management for patients with type $2 \mathrm{DM}$, as regards both stress levels and glycemic control. It is recommended to consider this type of treatment as an adjunct to conventional therapy. We deem that our study could encourage future studies in this area with larger samples, longer duration and more objective measurements.
\end{abstract}

Key words: Diabetes type 2, HbA1c, Intervention, Perceived stress, RCT, Stress management

Address for correspondence:

Efi Koloverou, MSc, PhD (candidate), 107 Sapfous Str.,

17676 Kallithea, Athens, Greece, Tel.: +302109532182,

E-mail: efi.koloverou@gmail.com

Received 18-10-2013, Accepted 18-02-2014

\section{INTRODUCTION}

Diabetes mellitus is a heterogeneous group of metabolic diseases characterized by chronic hyperglycemia and metabolic disorders, with type 2 diabetes 
being the most common form. ${ }^{1}$ Obesity and physical inactivity were identified early on as significant causal factors ${ }^{1-3}$ but only recently has stress been implicated in its pathology as well. Chronic activation of the hypothalamic pituitary axis (HPA) can lead to severe metabolic consequences, common in visceral obesity and metabolic syndrome. ${ }^{4-7}$ At the same time, diabetes itself can add to the initial stress burden not only due to the actual level of glycemia but also because of the pressure incurred by lifestyle modification and the insulin injection.

Therefore, many studies have tried to assess whether stress management can have a beneficial role in the management of patients with type 2 diabetes. Regarding stress reduction, the few available studies show that cognitive behavioral therapy (CBT), ${ }^{8,9}$ meditation and biofeedback appear to be effective. ${ }^{10,11}$ In terms of improving glycemic control, results have been mixed: some have found a positive effect, ${ }^{9-11}$ while others have not. ${ }^{8-12}$

One of the most popular stress management techniques is progressive muscle relaxation (PMR), ${ }^{13}$ which incorporates both a physical and a mental component. The knowledge of relaxation breathing (RB) is usually required for proper application. According to research data, the combination of this technique (RB-PMR) with others (biofeedback, guided imagery, CBT) has shown a beneficial effect in lowering stress levels, ${ }^{8,9,14}$ though a positive metabolic action has not yet been demonstrated. ${ }^{10,12}$

In summary, it is clear that the literature is as yet inadequate and the results of the few available studies mixed, especially regarding the effect of this technique on glycemic control. While there is still no systematic review of randomized clinical trials that have used PMR-RB to improve glycemic control in patients with type 2 diabetes, at the same time its combination with other techniques makes it difficult to draw conclusions about its effectiveness.

The primary aim of this study is to add to the literature by investigating the effects of RB-PMR on HbA1c, perceived stress (PSS) and health locus of control (HLC subscales). Secondary endpoints include the identification of changes in stress related symptoms, saliva cortisol, a putative dose-response effect, stress predictors and factors affecting compliance.

\section{SUBJECTS AND METHODOLOGY}

This was a parallel group, randomized, controlled trial with an almost 1:1 allocation ratio of treatment or non-treatment groups and 8 weeks of follow-up. After trial commencement there were a few deviations from original protocol (e.g. exclusion criteria, patient contact frequency).

The study was conducted in cooperation with the outpatient Diabetes Clinic of Laiko General Hospital in Athens, Greece, between November 2011 and July 2012. Recruitment was performed on the same days twice per week, from November 2011 till May 2012. All participants were fully informed about the purposes of the study and provided written consent. The study had the approval of the Scientific Committee of our hospital. Meetings with patients were one-on-one and were held in a specific area ceded to the researcher after an agreement between the Diabetes and Dietetics Departments. Eligible participants were all patients diagnosed with type 2 diabetes according to the criteria of the American Diabetes Association, who lived in Athens, were aged 30-75 years and were under the conventional treatment (diet, exercise, antidiabetic drugs and insulin). Exclusion criteria included diagnosis of type 1 diabetes mellitus, current neuroleptic or psycho-stimulant medication or psychiatric treatment, the use of corticosteroids within the last month, practice of other relaxation technique(s), significant acute or chronic concomitant disease, serious diabetes complications (e.g. severe visual impairment), end-stage renal disease and amputation, poorly controlled diabetes [glycated hemoglobin A1c (HbA1c)] > 8.5\%, $(>68 \mathrm{mmol} / \mathrm{mol})$ ], pregnancy, breastfeeding and incompetence at reading or writing in Greek. After enrollment, participants who changed their type or dose of diabetes medication or insulin or experienced an objectively severe stressful event (one or more of the first five events of the Social Readjustment Rating Scale) were also excluded from the analysis to avoid bias.

After an initial screening of each patient's file information, they were contacted and informed about the study. For those patients who declined to participate, the reasons were recorded. Patients who were interested to learn more were invited to the hospital for the first meeting so as to confirm eligibility and 
describe study requirements. Eligible participants were randomly assigned to either the intervention or the control group. A simple randomization procedure (random numbers generated by an online generator, www.random.org) was used. Randomization, baseline and final measurements were not blinded. On the other hand, data analysis was blinded. All information (e.g. effects of stress on health, instructions for saliva cortisol sample and questionnaires) were provided in an identical verbal and written manner. When the first questionnaires had been completed, $\mathrm{HbA1c}$ was measured and a saliva cortisol sample was taken at the second meeting. In the treatment group, RB-PMR was provided by an audio CD comprising 10 min of DB (deep breathing) and 15 min of PMR. Practice of some exercises and explanation of the RB-PMR philosophy and health benefits followed. All patient questions were answered. Patients were encouraged to focus on the difference between stress and relaxation through different muscle groups so as to increase their perception of relaxation response. ${ }^{13}$ In addition, they were asked to practice the technique twice a day for 8 weeks and keep a diary. When the technique was not performed, reasons were recorded. To measure and enhance compliance and eliminate dropouts, participants in the intervention group were contacted on a weekly basis. During the telephone communication, patients were asked to report on any problems when practicing RB-PMR and the principal sources of stress during the last week as well as to make general comments, all of which were recorded. No counseling was provided during the telephone communication. At the end of the intervention 8 weeks later, the final questionnaires and cortisol samples were returned and $\mathrm{HbA} 1 \mathrm{c}$ was measured. Finally, the control group continued with the usual care of the hospital department (physician and dietitian meeting) and at the end of the intervention participants were rewarded with a relaxation $\mathrm{CD}$.

Baseline and outcome measures included the following:

Sociodemographic characteristics (sex, age, marital status, educational level (years of education), place of residence (now and in the past), job schedule and self-reported financial status.

Medical history: Diabetes duration, type and dose of medication or insulin, occurrence of hyperlipidemia, hypertension or thyroid disease, other serious health problems, smoking status.

Lifestyle disease related factors: Body mass index (BMI), waist-to-hip ratio (WHR), physical activity status (type, frequency, intensity), adherence to the Mediterranean diet using the MedDietScore. ${ }^{15}$

Stress levels: The Perceived Stress Scale is a measure of the degree to which an individual's situations in life are appraised as stressful. ${ }^{16}$ Questions are designed in such a way that a person may evaluate how unpredictable, uncontrollable and overloaded he perceives his life to be. At the same time, there are questions that evaluate the levels of experienced stress directly. Participants were also asked about stress symptoms, both physical and psychological, through a specific list. Finally, the Social Readjustment Rating Scale (SRRS) ${ }^{17}$ consisting of 43 life events which are considered stressful, was completed. Each event has a numerical value. As mentioned above, the scale was used as a criterion for exclusion from the statistical analysis for patients who had experienced one of the first five listed events over the past 2 months.

Health Locus of Control (HLC subscales) ${ }^{18}$ validated in Greek. ${ }^{19}$ Individuals express their degree of agreement to 18 proposals on a 6-grade Likert scale. The scale consists of three subscales, independent of each other. The internal subscale reflects the degree to which a person believes that he/she is responsible for his/her health. The external subscale and the luckrelated subscale represents the extent to which other people (e.g. doctors, family) or luck, respectively, are perceived as the main determinants of health. The higher the score on each subscale, the stronger the belief in that determinant.

Laboratory data: HbA1c, an indicator of metabolic control for the past 3 months, was measured with high pressure liquid chromatography (HPLC). Saliva cortisol samples were also taken to determine stress levels. The secretion of cortisol is pulsed (about 15 beats a day) and its concentration in the blood fluctuates daily. The highest level occurs 30-45 min after awakening and is noted on the curve as CAR (Cortisol Awakening Response). Subsequently, a gradual decline can be observed until the evening, this reduction rate being described as the daily slope 
(diurnal slope). The average daily concentration of cortisol throughout the day is described by the area under the curve (Area Under the Curve-AUC). In this study we used the shortest protocol, which requires three measurements per day and has the largest application and reliability. The measurements were made immediately after awakening, and 45 minutes and 12 hours later, respectively (e.g. 08:00, 08:45 and 20:00).

For sample size calculation, we assumed a large attrition rate of $30 \%$ by week 8 during the intervention phase, primarily due to increased daily requirements. Therefore, we had anticipated needing at baseline 74 subjects (52 after dropouts) with type 2 diabetes to detect large effect sizes (effect size of $0.8 \mathrm{SD}$ ) with at least $80 \%$ power (statistical significance level $\mathrm{a}=0.05$, allocation ratio almost 1:1). Following enrollment, the dropout exclusion rate at the stage of analysis was $28 \%$, yielding a final sample of 53 patients.

For the presentation of the research data, the means \pm standard deviation was used for continuous variables as well as the absolute and proportion frequencies for categorical variables. For group comparisons, Student's t-test and the Mann-Whitney U-test were used, according to normality (assessed by Q-Q plots, histograms and the Kolmogorov-Smirnov or Shapiro-Wilk test), for continuous variables and the Chi-squared test for qualitative variables. Next, a modified per-protocol analysis was chosen because it contains less error compared to intention-to-treat analysis. Specifically, dropout reasons were about the same between the two groups and patients that were excluded from the analysis by the investigator reported severe stressors, which would have introduced bias.

Finally, in terms of statistical analysis, changes in HbA1c, PSS, HLC1, HLC2 and HLC3 scores were used as primary outcomes. To detect differences between groups, one-way analysis of covariance (ANCOVA) was used. All assumptions were checked. For PSS, HLC1, HLC2 and HLC3, the baseline values were used as covariates. For $\mathrm{HbA} 1 \mathrm{c}$, age, gender and baseline HbA1c were considered the most important covariates. PSS was not entered into the model due to moderate correlations with age (Spearman's rho $=-0.294, p=0.036)$ and sex (Spearman's rho $=0.312, p=0.026$ ), which induces undesir- able colinearity. Effect sizes were calculated for each variable using Cohen's $d$. Generally, the effect sizes of $0.8,0.5$ and 0.2 are considered as large, medium and small, respectively. Secondary endpoints (changes in symptoms perception, dose-response effect, factors affecting compliance with the technique, saliva cortisol levels) were addressed with simple Spearman's @ correlation tests and the parametric Student's t-test or non-parametric Mann-Whitney test, for numericalby-numerical and numerical-by-nominal comparisons, respectively. The level of statistical significance was $5 \%$. Statistical analysis was performed using SPSS for Windows (version 18.0.3) statistical software (SPSS Inc., Chicago, IL).

\section{RESULTS}

The study was conducted in 53 patients with type 2 diabetes referred to the outpatients' Diabetes Clinic. The flowchart of the participants is displayed in Figure 1.

Table 1 shows the distribution of the sociodemographic, clinical and lifestyle characteristics of the participants. For categorical variables, absolute and relative frequencies (n, \%) are used. For quantitative values, means or medians and standard deviations, depending on normal distribution assumption, are computed, respectively. Most of the participants were over 60, without a partner, overweight or obese, physically active, of moderate socioeconomic status, and none were current smokers. The majority took tablets rather than insulin. The mean baseline of PSS, HLC, cortisol levels and HBA1c are also presented. There were no significant baseline differences between the two study groups $(p>0.05)$.

A significant decrease in PSS was observed in the intervention group with moderate effect size (Cohen's $d=0.71, p=0.011$ ), after controlling for baseline PSS and interaction with the group (Table 2). After a visual graph assessment (Figure 2), it was apparent that the higher the initial levels of perceived stress, the greater the benefit of the technique for PSS scores $>16$. For PSS $<16$, there were not sufficient subjects to detect any potential relationship and this may be the reason for the interaction. As for $\mathrm{HbA} 1 \mathrm{c}$, moderate reduction with medium effect size (Cohen's $d=0.73, p=0.015$ ) was observed after adjustment for 


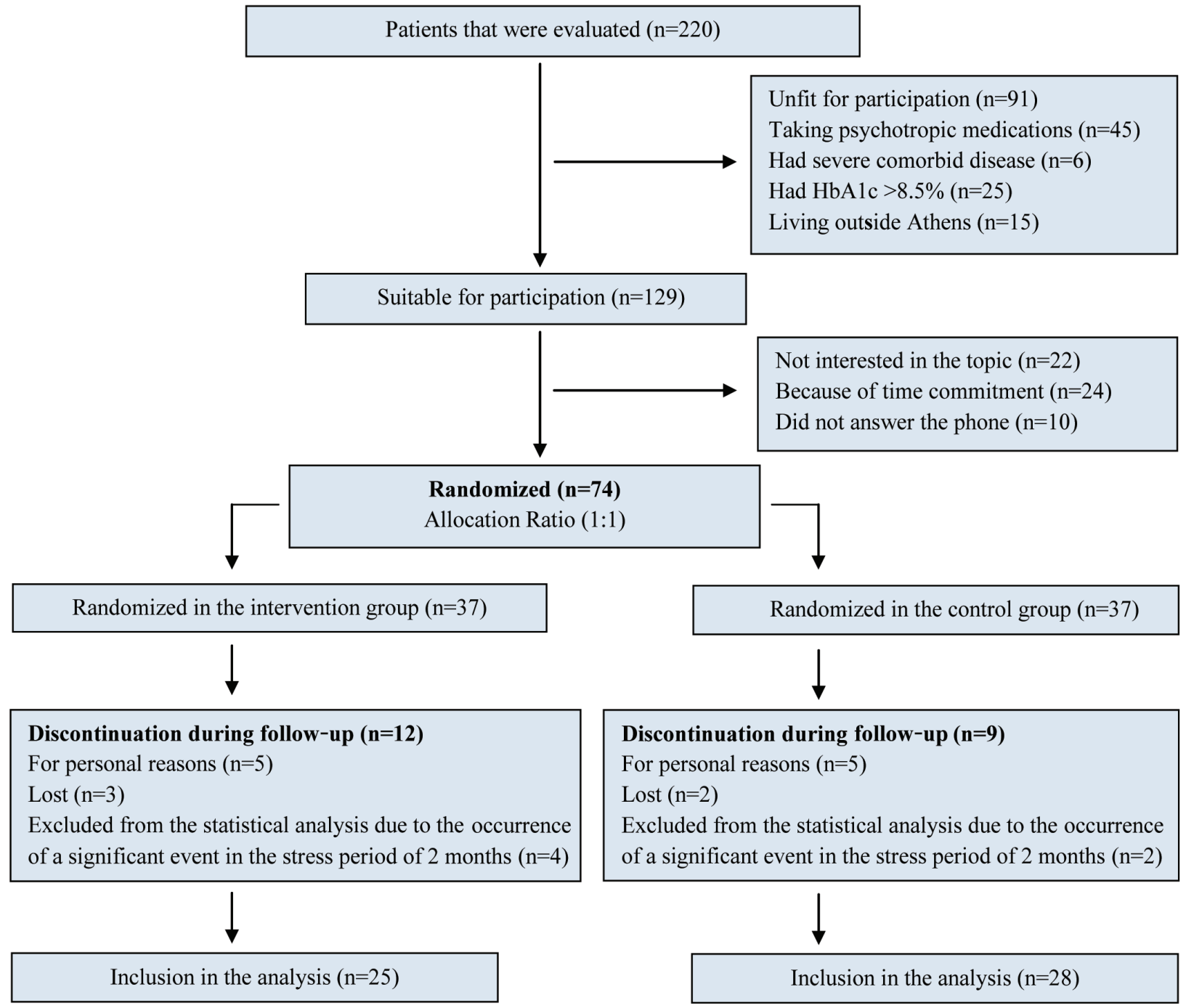

Figure 1. Flowchart of participants.

age, gender, baseline HbA1c levels and age by group interaction. The interaction indicates that the mean HbA1c differences depended on patients' age. As depicted in Table 2, the mean HbA1c change was calculated for patients of the same sex, aged 61.8 years and baseline $\mathrm{HbA1c} 6.7 \%$. A further analysis was conducted, adding socioeconomic status as a covariate, and $\mathrm{HbA} 1 \mathrm{c}$ reduction remained statistically significant $(p=0.029)$ (data not shown). For HLC subscales, small non-significant differences were observed after controlling for baseline values and interactions between baseline levels and group.

Regarding secondary endpoints, a decrease in the average stress symptoms score was observed as well as in physical and psychological symptoms subscores. However, only the physical symptoms score mean change was significant $(\Delta$ Phys score $=-3.25 \pm 6.11$, $p=0.023$ ).

Next, cortisol profiles in the whole sample and in each group separately before and after the intervention were studied. At the beginning of treatment, the cortisol profile was not consistent with the normal daily variation (circadian pattern) in the whole sample and the intervention group. Post intervention, cortisol followed the familiar pattern of variation, with higher values seen in the morning and 30-45 minutes after the alarm in both groups. However, no statistically significant difference was recorded. 
Table 1. Baseline sample characteristics

\begin{tabular}{|c|c|c|c|}
\hline & Intervention group & Control group & $p$ \\
\hline $\mathrm{N}(\%)$ & $25(47)$ & $28(53)$ & \\
\hline Age (years) & $60.52( \pm 6.73)$ & $63.00( \pm 8.00)$ & 0.230 \\
\hline Male sex, $\mathrm{N}(\%)$ & $17(32)$ & $28(53)$ & 0.999 \\
\hline Married, N (\%) & $18(34)$ & $24(45)$ & 0.313 \\
\hline Socioeconomic level* & & & 0.069 \\
\hline - low & $4(16.7)$ & $13(48.1)$ & \\
\hline - moderate & $16(66.7)$ & $13(48.1)$ & \\
\hline - high & $4(16.7)$ & $1(3.7)$ & \\
\hline Current smoking, N (\%) & $3(12)$ & $6(21.4)$ & 0.353 \\
\hline MedDietScore & $35.72( \pm 5.24)$ & $35.57( \pm 3.76)$ & 0.905 \\
\hline Physical activity frequency & & & 0.290 \\
\hline - Never & $3(12)$ & $8(28.6)$ & \\
\hline - Rare & $1(4)$ & $3(10.7)$ & \\
\hline -1-2 times/week & $5(20)$ & $3(10.7)$ & \\
\hline - >3 times/week & $16(64)$ & $14(50)$ & \\
\hline Body mass index $\left(\mathrm{kg} / \mathrm{cm}^{2}\right)^{1}$ & $29.3( \pm 7.1)$ & $31.9( \pm 6.3)$ & 0.178 \\
\hline Duration of diabetes (official diagnosis) (years) ${ }^{1}$ & $3.4( \pm 7.4)$ & $8( \pm 6.4)$ & 0.538 \\
\hline Antidiabetic tablets, N (\%) & $19(76)$ & $24(85.7)$ & 0.451 \\
\hline Insulin treated, $\mathrm{N}(\%)$ & $8(32)$ & $10(35.7)$ & 0.776 \\
\hline Family history of diabetes & $18(34)$ & $21(40)$ & 0.999 \\
\hline PSS score & $27.21( \pm 8.02)$ & $24.67( \pm 9.03)$ & 0.296 \\
\hline \multicolumn{4}{|l|}{ Health locus of control } \\
\hline - HLC-1 score ${ }^{1}$ & $28.96( \pm 5.27)$ & $28.12( \pm 5.83)$ & 0.844 \\
\hline - HLC-2 score ${ }^{1}$ & $17.21( \pm 7.93)$ & $18.42( \pm 7.17)$ & 0.412 \\
\hline - HLC-3 score ${ }^{1}$ & $27.58( \pm 6.12)$ & $27.65( \pm 6.38)$ & 0.992 \\
\hline \multicolumn{4}{|l|}{ Cortisol levels (mg/dl) } \\
\hline - After awakening ${ }^{1}$ & $0.71( \pm 0.30)$ & $0.62( \pm 0.41)$ & 0.831 \\
\hline - 45 minutes later ${ }^{1}$ & $0.56( \pm 0.29)$ & $0.59( \pm 0.37)$ & 0.915 \\
\hline - 12 hours later ${ }^{1}$ & $0.25( \pm 0.13)$ & $0.26( \pm 0.12)$ & 0.972 \\
\hline$-C A R^{* 1}$ & $-0.012( \pm 0.299)$ & $0.048( \pm 0.349)$ & 0.599 \\
\hline - Slope ${ }^{1}$ & $-0.028( \pm 0.023)$ & $-0.023( \pm 0.031)$ & 0.755 \\
\hline$-A U C^{1}$ & $4.96( \pm 1.8)$ & $4.64( \pm 2.7)$ & 0.929 \\
\hline $\operatorname{HbA1c}(\%) /(\mathrm{mmol} / \mathrm{mol})$ & $6.51( \pm 0.63) / 52( \pm 5)$ & $6.86( \pm 0.66) / 55( \pm 5)$ & 0.061 \\
\hline
\end{tabular}

PSS: Perceived Stress Scale, HLC: Health Locus of Control (1=internal, $2=$ luck, $3=$ external), HbA1c: glycosylated hemoglobin A1c, CAR: Cortisol Awakening Response, AUC: Area Under the Curve.

*The socioeconomic level was created as a variable from the responses of individuals as to whether household income meets their needs (not at all, slightly, moderately, considerably or greatly).

${ }^{1}$ These quantitative variables do not follow the normal distribution in at least one of the comparison groups. For these variables, data are presented as medians \pm SDs, and the Mann-Whitney U-test was applied. For the remaining quantitative variables, means \pm SD and Student's t-test were used, whereas for categorical variables Pearson's chi-square or Fisher's exact test was used.

Spearman's correlations of times of practice and primary outcomes were not significant (Spearman's rho $=0.123$ for $\Delta \mathrm{PSS}, 0.085$ for $\Delta \mathrm{HbA} 1 \mathrm{c}, 0.194$ for $\Delta \mathrm{HLC1}, 0.245$ for $\Delta \mathrm{HLC} 2,0.166$ for $\Delta \mathrm{HLC} 3)$. Thus, no dose-response relationship was observed. As far as compliance with treatment is concerned, 
Table 2. Adjusted mean changes $( \pm \mathrm{SD})$ of primary outcomes by study groups and effect sizes

\begin{tabular}{lcccc}
\hline Outcome & Intervention group $(\mathbf{n}=\mathbf{2 5})$ & Control group $(\mathbf{n}=\mathbf{2 8})$ & $p$ & Effect size $($ Cohen's $d)$ \\
\hline (D) PSS \pm PLACE & $-2.6( \pm 1.1)$ & $1.3( \pm 1.1)$ & $0.011^{*}$ & 0.71 \\
(D) HLC1 \pm SD Internal & $-10.7( \pm 1.77)$ & $-8.78( \pm 1.70)$ & 0.448 & 0.21 \\
(D) HLC2 \pm SD Chance & $0.59( \pm 1.25)$ & $1.45( \pm 1.20)$ & 0.511 & 0,14 \\
(D) HLC3 \pm SD Powerful others & $-0.14( \pm 0.709)$ & $0.01( \pm 0.681)$ & 0.883 & 0.04 \\
(D) HbA1c \pm SE & $-0.23( \pm 0.10)$ & $0.11( \pm 0.09)$ & $0.015 *$ & 0.73 \\
\hline
\end{tabular}

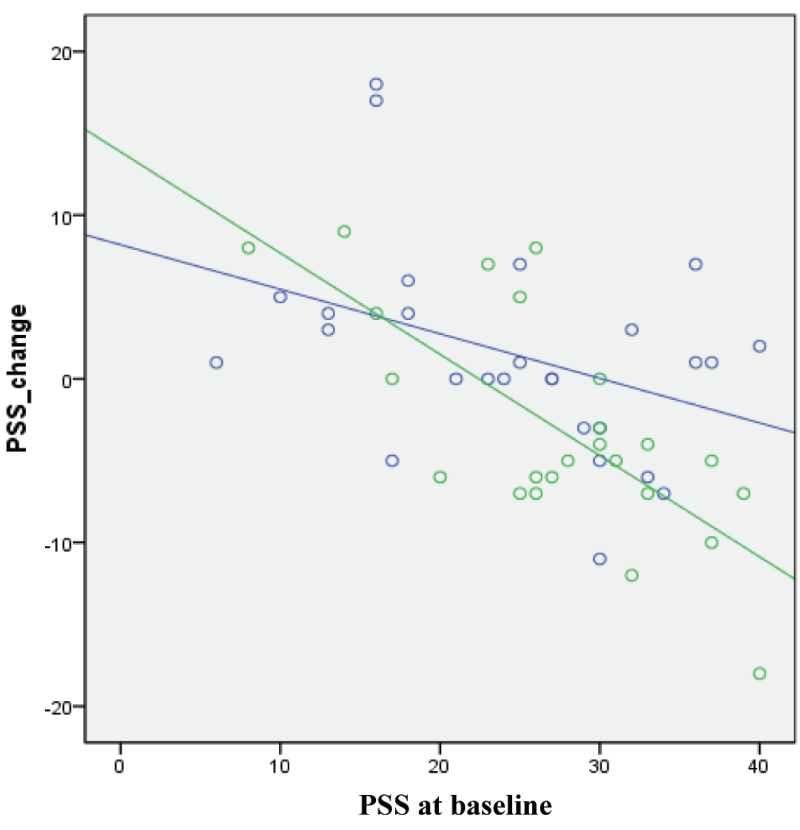

Intervention vs. Control group

Control group

Intervention group

Control group

Intervention group

Control group: $\mathrm{R}^{2}$ Linear $=0.15$

Intervention group: $\mathrm{R}^{2}$ Linear $=0.526$

Figure 2. Correlation between changes in Perceived Stress Score between the two study groups. Patients with higher baseline stress levels practicing RB-PMR for 8 weeks showed a larger reduction in PSS.

only individuals with increased BMI (Spearman's rho $=0.425, p=0.035$ ) and shorter disease duration (Spearman's rho $=-0.463, p=0.034$ ) were linearly associated with better compliance with relaxation treatment instructions.

Finally, it was examined whether certain variables (age, sex, level of education, use of insulin, diabetes history, socioeconomic level, number of children, body mass index, HbA1c, duration of diabetes mel- litus, occupation, smoking habits) were associated with greater levels of stress. We found that men (Spearman's rho $=-0.312, p=0.026$ ) and patients of higher socioeconomic status Spearman's rho $=-0.314$, $p=0.025$ ) experienced less stress in relation to women and people of lower socioeconomic level, respectively.

\section{DISCUSSION}

This randomized clinical trial was conducted to evaluate the effect of stress management through an 8 -week relaxation program. Our results can be summarized as the following: RB-PMR significantly reduced perceived stress and glycosylated hemoglobin with medium effect sizes recorded (Cohen's $d=0.71$ and 0.67 , respectively). In addition, higher baseline perceived stress was associated with greater PSS decrease. The three HLC subscales did not change significantly. Regarding stress symptoms, the physical, but not psychological, symptoms score decreased in the intervention group with moderate effect size (Cohen's $d=0.66$ ). In addition, patients with diabetes in the intervention group practiced the relaxation technique 0.8 times per day. Patients with a higher $\mathrm{BMI}$ and the most recent diabetes diagnosis showed better compliance with the program. However, no dose-response relationship between times of practice and primary outcomes was noted. Furthermore, female gender and low socioeconomic level were linearly associated with higher perceived stress at the beginning of the intervention. Finally, the baseline cortisol profile was blunted in the intervention group; after intervention, the cortisol profile was normal in both groups. Mean cortisol changes were not statistically significant.

Regarding the effect of RB-PMR on stress levels in patients with type 2 diabetes, there are no recent research data. However, previous studies suggest 
that this technique, when combined with biofeedback and CBT, has beneficial effects on stress and anxiety levels. ${ }^{8-14}$ On the other hand, when provided along with guided imagery it does not seem to offer similar benefits. ${ }^{12}$ Perceived stress reduction is likely to be mediated by the short-term effects of RB-PMR, such as a reduced perception of pain, a pleasant mental status, stimulation of the parasympathetic system or a temperature increase.$^{20}$ Long-term application has been associated with a decrease in cortisol saliva, generalized anxiety, ${ }^{21}$ blood pressure ${ }^{21,22}$ heart rate ${ }^{40}$ and headaches. ${ }^{23}$

As far as the benefits of the technique in glycemic control are concerned, few studies have used this technique in individual sessions. Therefore, a "side by side" comparison with our own study is difficult. In agreement with our study, Surwit et al found that PMR in combination with CBT and diabetes education during five group sessions can reduce $\mathrm{HbA1c}$ by $0.5 \%(4 \mathrm{mmol} / \mathrm{mol}){ }^{9}$ However, a previous study that used PMR and CBT during six group sessions did not find a statistically significant reduction in HbA1c. ${ }^{8}$ In another intervention in African women with type $2 \mathrm{MD}$, PMR was found to offer benefits similar to those of physical activity. ${ }^{24}$ Finally, some studies suggest that any benefits regarding glycemic control may not be detectable until after one year of intervention, ${ }^{9}$ or they may be restricted to specific groups of patients (i.e. those with elevated baseline anxiety, neuroticism, etc). ${ }^{12,25}$ In our study we did not test this particular case.

The effect of stress on metabolic activity has been thoroughly examined. Chronic activation of the HPA axis induces the release of count regulatory hormones (glucagon, epinephrine, cortisol), which affect the metabolic actions of insulin and lead to hyperglycemia. ${ }^{26,27}$ Moreover, cortisol, accompanied by an energy surplus, promotes mainly visceral obesity, which contributes to insulin resistance..$^{27}$ Another mechanism implicates inflammation. Stress triggers an acute phase inflammatory response, a key component of the immune response, which is mediated by macrophages, the liver, adipose tissue, etc. and leads to cytokines overproduction (IL-1, IL-6, TNF-a). ${ }^{43}$ Finally, stress induces ischemia of the gastrointestinal tract, leading to increased intestinal permeability and endotoxemia, which aggravates existing inflammation. ${ }^{28,29}$
Regarding secondary outcomes, relief of physical symptoms can selectively be attributed to the nature of the technique, which is more muscle-oriented and includes no cognitive or behavioral elements. ${ }^{30}$ Furthermore, in agreement with other studies, female gender ${ }^{31,32}$ and low socioeconomic status ${ }^{33}$ were associated with higher PSS levels at the beginning of the intervention. Finally, the initially blunt cortisol profile in the intervention group is consistent with the findings of Bruehl et al. ${ }^{34}$ However, this condition did not last till the end of the intervention and changes in mean cortisol were not statistically significant.

It is acknowledged that this study has a number of limitations. First, our primary outcomes, PSS and HLC, were based on self-reports as opposed to clinical and/or laboratory assessments. Especially regarding PSS, there are a number of potential confounders (e.g. personality, psychopathology, beliefs, values and the current mood of the respondents) that can seriously modify the results. ${ }^{35}$ Furthermore, most secondary outcomes, like symptoms of stress, were based on non-validated questionnaires, which can introduce information bias as they may not measure what they are created for and disregard important elements of the population for which they are designed. In addition, the intervention group generally showed greater consistency to questionnaire instructions and were more prone to improve final results (e.g. misjudge stress symptoms, perceived stress). The lack of blindness and the inability to confirm compliance aside from the diary entries should not be ignored. Another important point is the fact that the study duration was based on the technique protocol (8 weeks). However, $\mathrm{HbA1c}$ is commonly tracked after a 12-week interval, so it is possible that the actual HbA1c change is not entirely depicted at week 8 . Finally, there are no clinically meaningful cut-offs for perceived stress and secondary outcomes, thus the extrapolation of our results to clinical practice warrants caution. For this reason, sample size calculation was based solely on detecting high effect sizes.

The generalization of our results is limited to outpatients with type 2 diabetes without acute or severe coexisting disease, who live in an urban area and follow typical diabetes treatment. There are many reasons why this group of patients was chosen. Firstly, the technique requires the use of muscle groups; there- 
fore, patients with limited motor activity (i.e. patients with peripheral neuropathy or vascular disease) were excluded. Secondly, only patients following a stable treatment plan were included (mean disease duration 8 years). By that time, current treatment (diet, medication) will have conferred its benefits, thus, the addition of a supplementary treatment is rational. On the other hand, patients with newly diagnosed diabetes were excluded from the study due to frequent medication or insulin adjustments.

Overall, this study provides additional evidence of the benefits of stress management for patients with type 2 diabetes on both physical and psychological levels. Further research with larger samples, longer duration and more objective outcomes are necessary to assess the utility of relaxation techniques. We deem that the implementation of simple techniques, such as RB-PMR, characterized by low cost, small time requirements and feasibility, should be considered a cost-effective, non-pharmaceutical adjunct treatment for patients with type 2 diabetes in everyday clinical practice.

\section{FUNDING}

There was no funding of this study.

\section{CONFLICT OF INTEREST}

None declared.

\section{REFERENCES}

1. The InterAct Consortium, 2012 Physical activity reduces the risk of incident type 2 diabetes in general and in abdominally lean and obese men and women: the EPICInterAct Study. Diabetologia 55: 1944-1952.

2. Kohrt WM, Kirwan JP, Staten MA, Bourey RE, King DS, Holloszy JO, 1993 Insulin resistance in aging is related to abdominal obesity. Diabetes 42: 273-281.

3. Ohlson LO, Larsson B, Svardsudd K, et al, 1985 The influence of body fat distribution on the incidence of diabetes mellitus. 13.5 years of follow-up of the participants in the study of men born in 1913. Diabetes 34: $1055-1058$.

4. Wellen KE, Hotamisligil GS, 2005 Inflammation, stress, and diabetes. J Clin Invest 115: 1111-1119.

5. Friedman TC, Mastorakos G, Newman TD, et al, 1996 Carbohydrate and lipid metabolism in endogenous hypercortisolism: shared features with metabolic syndrome $\mathrm{X}$ and NIDDM. Endocr J 43: 645-655.
6. Kyrou I, Chrousos GP, Tsigos C, 2006 Stress, visceral obesity, and metabolic complications. Ann N Y Acad Sci 1083: 77-110.

7. Kyrou I, Tsigos C, 2007 Stress mechanisms and metabolic complications. Horm Metab Res 39: 430-438.

8. Henry J, Jane L, Wilson PH, Bruce DG, Chisholm DJ, Rawling DJ, 1997 Cognitive-behavioural stress management for patients with non- insulin dependent diabetes mellitus. Psychology, Health \& Medicine 2: 109-118.

9. Surwit RS, van Tilbur MA, Zucker N, et al, 2002 Stress management improves long-term glycemic control in type 2 diabetes. Diabetes Care 25: 30-34.

10. Rosenzweig S, Reibel DK, Greeson JM, et al, 2007 Mindfulness-based stress reduction is associated with improved glycemic control in type 2 diabetes mellitus: a pilot study. Altern Ther Health Med 13: 36-38.

11. McGinnis RA, McGrady A, Cox SA, Grower-Dowling KA, 2005 Biofeedback-assisted relaxation in type 2 diabetes. Diabetes Care 28: 2145-2149.

12. Aikens JE, Kiolbasa TA, Sobel R, 1997 Psychological predictors of glycemic change with relaxation training in non-insulin-dependent diabetes mellitus. Psychother Psychosom 66: 302-306.

13. Jacobson E, 1938 Progressive Relaxation. Chicago, University of Chicago Press.

14. Jablon SL, Naliboff BD, Gilmore SL, Rosenthal MJ, 1997 Effects of relaxation training on glucose tolerance and diabetic control in type II diabetes. Appl Psychophysiol Biofeedback 22: 155-169.

15. Panagiotakos DB, Pitsavos C, Stefanadis C, 2006 Dietary patterns: a Mediterranean diet score and its relation to clinical and biological markers of cardiovascular disease risk. Nutr Metab Cardiovasc Dis 16: 559-568.

16. Cohen S, Kamarck T, Mermelstein R, 1983 A global measure of perceived stress. J Health Soc Behav 24: 385-396.

17. Holmes TH, Rahe RH, 1967 The Social Readjustment Rating Scale. J Psychosom Res 11: 213-218.

18. Wallston KA, Wallston BS, DeVellis R, 1978 Development of the Multidimensional Health Locus of Control (MHLC) Scales. Health Educ Monogr 6: 160-170.

19. Karademas EC, 2009 Effects of exposure to the suffering of unknown persons on health-related cognitions, and the role of mood. Health (London) 13: 491-504.

20. Titlebaum HM, 1988 Relaxation. Holist Nurs Pract 2: $17-25$.

21. Pawlow LA, Jones GE, 2005 The impact of abbreviated progressive muscle relaxation on salivary cortisol and salivary immunoglobulin A (sIgA). Appl Psychophysiol Biofeedback 30: 375-387.

22. Sheu S, Irvin BL, Lin HS, Mar CL, 2003 Effects of progressive muscle relaxation on blood pressure and psychosocial status for clients with essential hypertension in Taiwan. Holist Nurs Pract 17: 41-47.

23. Anderson RE, Seniscal C, 2006 A comparison of selected 
osteopathic treatment and relaxation for tension-type headaches. Headache 46: 1273-1280.

24. van Rooijen AJ, Rheeder P, Eales CJ, Becker PJ, 2004 Effect of exercise versus relaxation on haemoglobin A1C in Black females with type 2 diabetes mellitus. QJM 97: 343-351.

25. Lane GD, McCaskill CC, Ross SL, Feinglos MN, Surwit RS, 1993 Relaxation training for NIDDM. Predicting who may benefit. Diabetes Care 16: 1087-1094.

26. Chrousos GP, 1998 Stressors, stress, and neuroendocrine integration of the adaptive response. The 1997 Hans Selye Memorial Lecture. Ann N Y Acad Sci 851: 311-335.

27. Morton NM, 2010 Obesity and corticosteroids: 11 betahydroxysteroid type 1 as a cause and therapeutic target in metabolic disease. Mol Cell Endocrinol 316: 154-164.

28. Yang RB, Mark M, Gray A, et al, 1998 Toll-like receptor-2 mediates lipopolysaccharide-induced cellular signalling. Nature 395: 284-288.

29. Deitch EA, 1992 Multiple organ failure. Pathophysiology and potential future therapy. Ann Surg 216: 117-134.

30. Lehrer PM, Carr R, Sargunaraj D, Woolfolk RL, 1994 Stress management techniques: are they all equivalent, or do they have specific effects? Biofeedback Self Regul 19: 353-401.

31. Barbosa-Leiker C, Kostick M, Lei M, et al, 2012 Measurement Invariance of the Perceived Stress Scale and Latent Mean Differences across Gender and Time. Stress Health 29: 253-260.

32. Herrero SG, Saldana MA, Rodriguez JG, Ritzel DO, 2012 Influence of task demands on occupational stress: gender differences. J Safety Res 43: 365-374.

33. Rebbeck TR, Weber AL, Spangler E, Zeigler-Johnson CM, 2013 What stresses men? predictors of perceived stress in a population-based multi-ethnic cross sectional cohort. BMC Public Health 13: 113.

34. Bruehl H, Wolf OT, Convit A, 2009 A blunted cortisol awakening response and hippocampal atrophy in type 2 diabetes mellitus. Psychoneuroendocrinology 34: 815-821.

35. Herbert TB, Cohen S, 1996 Measurement issues in research on psychosocial stress. In: HB Kaplan (ed) Psychosocial stress: Perspectives on structure, theory, life-course, and methods, Academic Press, London; pp, 295-332. 


\title{
Non-alcoholic fatty liver disease in women with polycystic ovary syndrome: assessment of non-invasive indices predicting hepatic steatosis and fibrosis
}

\author{
Stergios A. Polyzos, ${ }^{1}$ Dimitrios G. Goulis, ${ }^{2}$ Jannis Kountouras, ${ }^{1}$ Gesthimani Mintziori, ${ }^{2}$ \\ Panagiotis Chatzis, ${ }^{3}$ Efstathios Papadakis, ${ }^{3}$ Ilias Katsikis, ${ }^{3}$ Dimitrios Panidis ${ }^{3}$
}

${ }^{1}$ Second Medical Clinic, Ippokration Hospital; ${ }^{2}$ Unit of Reproductive Endocrinology, First Department of Obstetrics and Gynecology, Papageorgiou Hospital; ${ }^{3}$ Division of Endocrinology and Human Reproduction, Second Department of Obstetrics and Gynecology, Ippokration Hospital; Aristotle University of Thessaloniki, Thessaloniki, Greece

\begin{abstract}
OBJECTIVE: Insulin resistance contributes to the pathogenesis of both polycystic ovary syndrome (PCOS) and non-alcoholic fatty liver disease (NAFLD). The main aim of the present study was the evaluation of non-invasive indices of hepatic steatosis and fibrosis in PCOS women with or without metabolic syndrome (MetS). DESIGN: In this cross-sectional study, three non-invasive indices for hepatic steatosis [NAFLD liver fat score, lipid accumulation product (LAP) and hepatic steatosis index (HIS)] and four for fibrosis [FIB-4, aspartate aminotransferase (AST)-to-Platelet Ratio Index (APRI), body mass index (BMI)-Age-Alanine aminotransferase (ALT)-Triglycerides (BAAT) and BMI AST/ALT Ratio Diabetes (BARD)] were calculated in 314 PCOS women ( 77 with, 237 without MetS) and 78 controls. RESULTS: All steatosis indices were significantly higher in the PCOS than the control group (NAFLD liver fat score: $-0.139 \pm 0.117$ vs. $-0.976 \pm 0.159, p<0.001$; LAP: $43.3 \pm 1.9$ vs. $34.7 \pm 3.1, p=0.036$; HIS: 44.6 \pm 0.5 vs. $42.1 \pm 0.8, p=0.016)$. FIB-4 and BAAT [fibrosis stage $(F) 2-4$ ] were higher in the PCOS group $(0.480 \pm 0.020$ vs. $0.400 \pm 0.013, p<0.001$; and $15.6 \%$ vs. $5.1 \%$, respectively $)$ whereas APRI and BARD were not. All steatosis indices were significantly higher in PCOS women with than without MetS (NAFLD liver fat score: $1.874 \pm 0.258$ vs. $-0.793 \pm 0.099, p<0.001$; LAP: $76.8 \pm 4.9$ vs. 33.4 $\pm 1.4, p<0.001$; and HIS: $49.8 \pm 1$ vs. $43 \pm 0.5, p<0.001)$. Of the fibrosis indices, only BAAT (F2-4: $50.6 \%$ vs. $4.2 \%$ ) was higher in PCOS women with MetS. CONCLUSIONS: Non-invasive indices of hepatic steatosis were significantly higher in PCOS, especially in the presence of MetS, whereas indices of hepatic fibrosis yielded controversial results. Further studies are warranted to evaluate the long-term outcomes of hepatic steatosis and fibrosis indices in PCOS women.
\end{abstract}

Key words: APRI, FIB-4, Hepatic steatosis index, Insulin resistance, Lipid accumulation product, Metabolic syndrome, NAFLD liver fat score, Steatosis 


\section{INTRODUCTION}

Polycystic ovary syndrome (PCOS) is the commonest endocrine disorder in women of reproductive age, ${ }^{1}$ its prevalence having been reported to be up to $18 \%$ in accordance with the ESHRE/ASRM criteria. ${ }^{2}$ Multiple metabolic aberrations, such as insulin resistance (IR) and hyperinsulinemia, visceral obesity, inflammation and endothelial dysfunction, hypertension, dyslipidemia, high incidence of impaired glucose tolerance and a lifetime risk of type 2 diabetes mellitus (T2DM) and cardiovascular diseases are associated with the syndrome. ${ }^{3}$

Non-alcoholic fatty liver disease (NAFLD), the commonest hepatic disorder and the leading cause of cryptogenic cirrhosis in Western countries, constitutes a major, global, public health problem. ${ }^{4}$ Its prevalence has been reported to be $10-46 \%$ and $6-35 \%$ in the US and the rest of the world, respectively, ${ }^{5}$ and it increases mortality mainly due to hepatic and cardiovascular disease. ${ }^{6}$

Although the pathogenesis of either disorder has not yet been fully elucidated, IR has been implicated, at least partly, in the pathogenesis of both diseases. ${ }^{2,7-8}$ IR has been proposed as the common pathogenetic mechanism in conditions clustered under the term of metabolic syndrome (MetS), including obesity, T2DM, dyslipidemia, hypertension, PCOS and NAFLD, all of which increase the risk for the abovementioned cardiovascular diseases and mortality, the endpoints of MetS. ${ }^{8}$ Based on the observation that IR is the common underlying factor, studies evaluating the association between PCOS and NAFLD are increasing and are systematically summarized elsewhere. ${ }^{9}$ It seems that women with PCOS are possibly at risk for developing NAFLD and, conversely, NAFLD may be a risk factor for PCOS. ${ }^{9}$ However, the majority of relevant studies to date are not based on liver biopsy, regarded as the gold standard for the diagnosis of NAFLD,${ }^{10}$ because it raises obvious ethical issues. Therefore, the use of non-invasive indices of hepatic injury is gaining increasing interest, as they offer an estimation of NAFLD stage and grade and thus may serve as accurate tools, at least for selection of patients for liver biopsy. ${ }^{11}$

The primary endpoints of this study were the evaluation of non-invasive indices of hepatic steatosis and fibrosis in women: a) with PCOS vs. without PCOS; and b) with PCOS associated or not with MetS. The secondary endpoint of this study was the association of non-invasive indices of hepatic steatosis and fibrosis with variables related to sex hormones or IR.

\section{PATIENTS AND METHODS}

\section{Patients}

This was a one-center, cross-sectional study. Data were prospectively collected based on protocols of previous studies, ${ }^{7,12-13}$ but retrospectively reviewed for this study. Premenopausal women with PCOS and apparently healthy female controls were recruited on an outpatient basis at the Gynecological Endocrinology Infirmary of the Second Department of Obstetrics and Gynecology, Aristotle University of Thessaloniki, Greece. Women with PCOS were referred to the Gynecological Endocrinology Infirmary for diagnostic evaluation and/or treatment; controls were healthy volunteers. All participants provided informed consent. The study protocol conformed to the ethical guidelines of the 1975 Declaration of Helsinki and was approved by the institutional review board.

The inclusion criteria for the PCOS group were based on the revised criteria of ESHRE/ASRM, which require the presence of at least two of the following three features: 1 ) oligo- or anovulation ( $<8$ spontaneous hemorrhagic episodes/year); 2) biochemical hyperandrogenemia [defined in our population as early follicular phase serum total testosterone $>2.1$ $\mathrm{nmol} / \mathrm{L}$, corresponding to the mean +2 standard deviations (SD) testosterone concentrations in 200 control subjects measured in our laboratory] or clinical manifestations of hyperandrogenemia; and 3) polycystic ovary morphology on ultrasound $(\geq 12$ small follicles in at least one ovary and/or ovarian volume $\left.>10 \mathrm{~cm}^{3}\right) .{ }^{2}$ According to this definition, PCOS can be subdivided into four different phenotypes of PCOS: 1) severe PCOS (when all three criteria are included); 2) oligo- or anovulation and hyperandrogenemia; 3 ) ovulatory PCOS (hyperandrogenemia and polycystic ovary morphology); and 4) mild PCOS (oligo- or anovulation and polycystic ovary morphology). ${ }^{1}$

The PCOS group was further subdivided based on the presence of MetS according to the definitions proposed by the International Diabetes Federation 
(IDF). The reason for choosing this definition was that the same definition was applied for the estimation of the NAFLD liver fat score, which is one of the non-invasive indices used in this study.

Exclusion criteria for both PCOS and control groups were: 1) congenital adrenal hyperplasia (a short Synachten test was performed in all women with basal serum $17 \alpha$-hydroxyprogesterone concentrations $>1.5 \mathrm{ng} / \mathrm{mL}$ ); 2) Cushing's syndrome; 3) galactorrhea; 4) androgen-secreting tumors; 5) ethanol consumption $>20 \mathrm{~g} /$ day; 6 ) history of liver cirrhosis or other liver disease (viral hepatitis, autoimmune hepatitis, primary sclerosing cholangitis, primary biliary cirrhosis, drug-induced liver disease, hemochromatosis, Wilson's disease, $\alpha 1$-antitrypsin deficiency); 7) type 1 diabetes mellitus; 8) uncontrolled hypothyroidism or hyperthyroidism; 9) adrenal insufficiency; 10) renal failure; 11) cancer; 12) pregnancy; 13) premature ovarian failure; 13 ) addiction to any drug; 14) use of the following medications within the last semester before screening: estrogens, androgens, anti-androgens, progestins, glucocorticosteroids, spironolactone, insulin, thiazolidinediones, ursodeoxycholic acid, ferrum, interferon, tamoxifene, amiodarone, biologic agents, any medication against tuberculosis, epilepsy or viruses, or any medication affecting hemostasis, such as antiplatelet agents or oral anticoagulants.

\section{Methods}

In all women, weight, height, waist circumference (WC) and hip circumference (HC) were measured. Baseline blood samples were collected after an overnight fast between days 3 and 7 of the menstrual cycle in the control group and after a spontaneous bleeding episode in the PCOS group. The serum concentrations of follicle stimulating hormone (FSH), luteinizing hormone $(\mathrm{LH})$, prolactin, total testosterone, $\Delta_{4}-$ androstenedione, dehydroepiandrosterone-sulphate (DHEAS), sex hormone-binding globulin (SHBG), insulin, glucose, total cholesterol, high-density lipoprotein cholesterol (HDL-C), triglycerides, uric acid, aspartate transaminase (AST), alanine transaminase (ALT), gamma-glutamyl transferase (GGT), alkaline phosphatase (ALP) and platelets count were measured. The methodology used for serum measurements has been previously described in detail. ${ }^{13}$ The same experienced sonographer performed transvaginal ultrasonography on the same day as the blood was drawn; the volume and the number of small follicles (measuring 2-9 $\mathrm{mm}$ in diameter) of each ovary were determined.

The diagnoses of T2DM or impaired fasting glucose (IFG) were based on the American Diabetes Association (ADA) definition; T2DM: either fasting plasma glucose $\geq 7 \mathrm{mmol} / \mathrm{L}$ or 2 -h plasma glucose $\geq 11 \mathrm{mmol} / \mathrm{L}$ during an oral glucose tolerance test (75 $\mathrm{g}$ anhydrous glucose dissolved in water), which was performed in all the participants; IFG: fasting plasma glucose 5.5-6.9 $\mathrm{mmol} / \mathrm{L}$.

Body mass index (BMI) was calculated by the formula: body weight $(\mathrm{kg}) /$ height $^{2}\left(\mathrm{~m}^{2}\right)$. The waist-to-hip ratio (WHR), LH-to-FSH ratio and AST-to-ALT ratio were also calculated. Low-density lipoprotein cholesterol (LDL-C) was estimated using the Friedewald formula. Free androgen index (FAI) was calculated by the formula: FAI $=$ total testosterone $(\mathrm{nmol} / \mathrm{L}) /$ SHBG (nmol/L) X 100. IR, $\beta$-cell function and insulin sensitivity were quantified by the homeostatic model of assessment (HOMA)-IR, HOMA- $\beta$ and quantitative insulin sensitivity check index (QUICKI), respectively, by the formulas: HOMA-IR = fasting glucose $(\mathrm{mmol} / \mathrm{L}) \mathrm{x}$ fasting insulin $(\mu \mathrm{U} / \mathrm{mL}) / 22.5$; HOMA $-\beta=[20 *$ fasting insulin $(\mu \mathrm{U} / \mathrm{mL})] /[$ fasting glucose $(\mathrm{mmol} / \mathrm{L})-3.5]$; QUICKI $=1 /[\log ($ fasting insulin; $\mu \mathrm{U} / \mathrm{mL})+\log ($ fasting glucose; $\mathrm{mg} / \mathrm{dL})$ ].

Based on the available data, three non-invasive indices for hepatic steatosis [NAFLD liver fat score, ${ }^{14}$ lipid accumulation product (LAP) ${ }^{15}$ and hepatic steatosis index $9 \mathrm{HIS}^{16}$ ] and four indices for hepatic fibrosis [FIB-4, ${ }^{17}$ AST-to-Platelet Ratio Index (APRI) ${ }^{18}$ BMI Age ALT Triglycerides (BAAT) ${ }^{19}$ and BMI Age ALT Triglycerides (BARD $)^{20}$ ] were estimated for all women with PCOS and controls. The required parameters and equations for each of these indices are presented in detail in Table 1.

\section{Statistical Analysis}

Continuous data were presented as mean \pm standard error of the mean (SEM). Categorical data were presented as frequencies. The Kolmogorov-Smirnov test was used to check the normality of distributions of continuous variables. The independent sample T-test or Mann-Whitney test was used for between-group 
Table 1. Formulas of non-invasive indices for hepatic steatosis and fibrosis used for the study

\begin{tabular}{|c|c|c|}
\hline Non-invasive index & Required parameters & Equation \\
\hline $\begin{array}{l}\text { NAFLD liver fat } \\
\text { score }\end{array}$ & $\begin{array}{l}\text { MetS }(\text { yes }=1 / \text { no }=0) \# \\
\text { T2DM }(\text { yes }=1 / \text { no }=0) \\
\text { Fasting serum insulin }(\mathrm{mU} / \mathrm{L}) \\
\text { AST }(\mathrm{U} / \mathrm{L}) \\
\text { AST/ALT }(\text { each in } \mathrm{U} / \mathrm{L})\end{array}$ & $\begin{array}{l}-2.89+1.18 \times(\mathrm{MetS})+0.45 \times(\mathrm{T} 2 \mathrm{DM})+0.15 \times(\text { fast }- \\
\text { ing serum insulin })+0.04 \times(\mathrm{AST})-0.94 \times(\mathrm{AST} / \mathrm{ALT})\end{array}$ \\
\hline LAP & $\begin{array}{l}\text { WC }(\mathrm{cm}) \\
\text { Triglycerides }(\mathrm{mmol} / \mathrm{L})\end{array}$ & For women: (waist circumference -58 ) $\times$ triglycerides \\
\hline HIS & $\begin{array}{l}\text { ALT/AST }(\text { each in U/L) } \\
\text { BMI }\left(\mathrm{kg} / \mathrm{m}^{2}\right) \\
\text { T2DM }(\text { yes }=1 / \text { no }=0) \\
\text { Female }(\text { yes }=1 / \text { no }=0)\end{array}$ & $8 \times(\mathrm{ALT} / \mathrm{AST})+\mathrm{BMI}+2 \times($ female $)+2 \times(\mathrm{T} 2 \mathrm{DM})$ \\
\hline FIB-4 & $\begin{array}{l}\text { Age (years) } \\
\text { AST }(\mathrm{U} / \mathrm{L}) \\
\operatorname{ALT}(\mathrm{U} / \mathrm{L}) \\
\text { Platelets }\left(\mathrm{N}^{*} 10^{3} / \mu \mathrm{L}\right)\end{array}$ & $($ Age $x$ AST $) /\left(\right.$ platelets $\times$ ALT $\left.^{1 / 2}\right)$ \\
\hline APRI & $\begin{array}{l}\operatorname{AST}(\mathrm{U} / \mathrm{L}) \\
\text { Platelets }\left(\mathrm{N}^{*} 10^{3} / \mu \mathrm{L}\right)\end{array}$ & $100 \times(\mathrm{AST} /($ upper normal limit of AST $)) /$ platelets \\
\hline BAAT & $\begin{array}{l}\text { Age } \geq 50 \text { years }(\text { yes }=1 / \mathrm{no}=0) \\
\mathrm{BMI} \geq 28 \mathrm{~kg} / \mathrm{m}^{2}(\text { yes }=1 / \mathrm{no}=0) \\
\text { Triglycerides } \geq 1.7 \mathrm{mmol} / \mathrm{L}(150 \mathrm{mg} / \mathrm{dL})(\text { yes }=1 / \mathrm{no}=0) \\
\text { ALT } \geq 2 \text { times upper normal limit }(\text { yes }=1 / \mathrm{no}=0)\end{array}$ & Age + BMI + triglycerides + ALT \\
\hline BARD & $\begin{array}{l}\mathrm{BMI} \geq 28 \mathrm{~kg} / \mathrm{m}^{2}(\mathrm{yes}=1 / \mathrm{no}=0) \\
\mathrm{T} 2 \mathrm{DM}(\mathrm{yes}=1 / \mathrm{\text {no }}=0) \\
\mathrm{AST} / \mathrm{ALT} \geq 0.8(\text { yes }=1 / \mathrm{no}=0)\end{array}$ & $\mathrm{BMI}+\mathrm{T} 2 \mathrm{DM}+2 \times(\mathrm{ALT} / \mathrm{AST})$ \\
\hline
\end{tabular}

\#According to International Diabetes Federation (IDF) definition.

ALT: alanine transaminase; APRI: AST to Platelet Ratio Index; AST: aspartate transaminase; BAAT: BMI Age ALT Triglycerides; BARD: BMI AST/ALT Ratio Diabetes; BMI: body mass index; HIS: hepatic steatosis index; LAP: lipid accumulation product; MetS: metabolic syndrome; NAFLD: non-alcoholic fatty liver disease; T2DM: type 2 diabetes mellitus; WC: waist circumference.

comparisons in cases of two groups of continuous variables. One-way analysis of variance (ANOVA) or the Kruskal-Wallis test (with Bonferroni post-hoc adjustment) was used for between-group comparisons in cases of more than two groups of continuous variables. Analysis of covariance (ANCOVA) was used to adjust between-group comparisons for covariates. The chi-square or Fisher exact test was used for between-group comparisons of categorical variables. Spearman's coefficient $\left(\mathrm{r}_{\mathrm{s}}\right)$ was used for binary correlations. Statistical analysis was performed with SPSS 21.0 for Macintosh (IBM Corp., Armonk, NY). Significance was set at a level of $\mathrm{p}<0.05$.

\section{RESULTS}

\section{Comparison between women with PCOS and controls}

Three hundred and fourteen women with PCOS and 78 controls were included in this study. The PCOS group was divided into: 1$)$ severe $(\mathrm{n}=164) ; 2)$ oligo- or anovulation and hyperandrogenemia $(\mathrm{n}=89)$; 3 ) ovulatory $(\mathrm{n}=30)$; and 4$)$ mild PCOS $(\mathrm{n}=31)$. Comparative data of the study groups are presented in Table 2 . The control group was of significantly higher age compared with the PCOS group. As expected, BMI, WC and $\mathrm{HC}$, testosterone, DHEAS, $\Delta_{4}$-androstenedione and FAI, LH, LH to FSH ratio were significantly higher, whereas SHBG and FSH were lower in the PCOS compared with the control group. Regarding liver function tests, ALP was significantly higher in the PCOS compared with the control group, whereas AST, ALT, GGT and AST to ALT ratio were similar between groups. Total and HDL-cholesterol were significantly higher in the control group, whereas LDL-C and triglycerides were not different between groups. Interestingly, serum uric acid was significantly higher in the PCOS compared with the control group. 
Table 2. Comparative data between PCOS and control group

\begin{tabular}{|c|c|c|c|c|}
\hline & Control group & PCOS group & p-value * & Reference range \\
\hline Women $(\mathrm{N})$ & 78 & 314 & - & - \\
\hline IFG $[\mathrm{N}(\%)]$ & $9(11.5)$ & $45(14.3)$ & 0.522 & - \\
\hline $\mathrm{T} 2 \mathrm{DM}[\mathrm{N}(\%)]$ & $1(1.3)$ & $6(1.9)$ & 0.707 & - \\
\hline $\operatorname{MetS}[N(\%)]^{\#}$ & $10(12.8)$ & $77(24.5)$ & 0.026 & - \\
\hline Age (years) & $33.0 \pm 0.5$ & $26.1 \pm 0.4$ & $<0.001$ & - \\
\hline BMI $\left(\mathrm{kg} / \mathrm{m}^{2}\right)$ & $28.8 \pm 0.7$ & $31.7 \pm 0.4$ & 0.001 & $20-25$ \\
\hline $\mathrm{WC}(\mathrm{cm})$ & $87.8 \pm 1.6$ & $93.9 \pm 0.9$ & 0.002 & $<80$ \\
\hline $\mathrm{HC}(\mathrm{cm})$ & $108.9 \pm 1.3$ & $114.9 \pm 0.2$ & $<0.001$ & na \\
\hline WHR & $0.803 \pm 0.008$ & $0.815 \pm 0.004$ & 0.201 & na \\
\hline Testosterone (nmol/L) & $1.21 \pm 0.05$ & $2.54 \pm 0.06$ & $<0.001$ & $0.5-2.1$ \\
\hline DHEAS $(\mu \mathrm{mol} / \mathrm{L})$ & $5.40 \pm 0.24$ & $7.86 \pm 0.20$ & $<0.001$ & $4.1-10.3$ \\
\hline$\Delta 4$-androstenedione $(\mathrm{nmol} / \mathrm{L})$ & $6.49 \pm 0.28$ & $9.81 \pm 0.24$ & $<0.001$ & $3.5-13.3$ \\
\hline $\mathrm{SHBG}(\mathrm{nmol} / \mathrm{L})$ & $60.6 \pm 3.6$ & $36.4 \pm 1.3$ & $<0.001$ & $30-135$ \\
\hline FAI & $2.4 \pm 0.1$ & $9.7 \pm 0.4$ & $<0.001$ & $<5$ \\
\hline Prolactin (pmol/L) & $517 \pm 30$ & $570 \pm 17$ & 0.077 & $130-1260$ \\
\hline $\mathrm{LH}(\mathrm{U} / \mathrm{L})$ & $5.9 \pm 0.5$ & $7.7 \pm 0.3$ & 0.001 & $2-12.5$ \\
\hline FSH (U/L) & $6.3 \pm 0.2$ & $5.8 \pm 0.1$ & 0.040 & $2.5-10$ \\
\hline LH to FSH ratio & $1.00 \pm 0.09$ & $1.38 \pm 0.05$ & $<0.001$ & na \\
\hline $\operatorname{AST}(\mathrm{U} / \mathrm{L})$ & $18.7 \pm 0.8$ & $20 \pm 0.5$ & 0.197 & $10-31$ \\
\hline $\operatorname{ALT}(\mathrm{U} / \mathrm{L})$ & $25.8 \pm 1.3$ & $26.9 \pm 0.9$ & 0.768 & $10-34$ \\
\hline AST to ALT ratio & $0.820 \pm 0.035$ & $0.893 \pm 0.026$ & 0.180 & na \\
\hline GGT (U/L) & $21.5 \pm 1.7(N=52)$ & $20.6 \pm 0.8(N=144)$ & 0.756 & $0-38$ \\
\hline $\operatorname{ALP}(\mathrm{U} / \mathrm{L})$ & $71.3 \pm 2.8$ & $76.9 \pm 1.2$ & 0.046 & $30-120$ \\
\hline Total cholesterol (mmol/L) & $5.26 \pm 0.13$ & $4.97 \pm 0.05$ & 0.038 & $<5.2^{\S}$ \\
\hline $\mathrm{HDL}-\mathrm{C}(\mathrm{mmol} / \mathrm{L})$ & $1.37 \pm 0.05$ & $1.29 \pm 0.03$ & 0.027 & $>1.3$ \\
\hline $\mathrm{LDL}-\mathrm{C}(\mathrm{mmol} / \mathrm{L})$ & $3.37 \pm 0.10$ & $3.16 \pm 0.05$ & 0.089 & $<4.1^{\S}$ \\
\hline Triglycerides (mmol/L) & $1.07 \pm 0.06$ & $1.11 \pm 0.03$ & 0.867 & $<1.7$ \\
\hline Uric acid $(\mu \mathrm{mol} / \mathrm{L})$ & $250 \pm 6$ & $286 \pm 6$ & $<0.001$ & $155-393$ \\
\hline Platelets $\left(\mathrm{N} x 10^{3} / \mu \mathrm{L}\right)$ & $278 \pm 9$ & $282 \pm 4$ & 0.655 & $140-400$ \\
\hline Glucose $(\mathrm{mmol} / \mathrm{L})$ & $5.44 \pm 0.06$ & $5.49 \pm 0.06$ & 0.603 & $3.3-5.6$ \\
\hline Insulin (pmol/L) & $82.7 \pm 5.6$ & $115.3 \pm 4.2$ & $<0.001$ & $42-188$ \\
\hline HOMA-IR & $2.92 \pm 0.21$ & $4.15 \pm 0.17$ & $<0.001$ & na \\
\hline HOMA- $\beta$ & $125 \pm 8$ & $179 \pm 8$ & $<0.001$ & na \\
\hline QUICKI & $0.335 \pm 0.003$ & $0.322 \pm 0.002$ & 0.001 & na \\
\hline NAFLD liver fat score & $-0.976 \pm 0.159$ & $-0.139 \pm 0.117$ & $<0.001$ & $\begin{array}{c}<-1.413: \text { no steatosis } \\
>1.257: \text { steatosis }\end{array}$ \\
\hline LAP & $34.7 \pm 3.1$ & $43.3 \pm 1.9$ & 0.036 & na \\
\hline HIS & $42.1 \pm 0.8$ & $44.6 \pm 0.5$ & 0.016 & $\begin{array}{c}<30: \text { no steatosis } \\
>36: \text { steatosis }\end{array}$ \\
\hline FIB-4 & $0.400 \pm 0.013$ & $0.480 \pm 0.020$ & $<0.001$ & $>1.3$ : advanced fibrosis \\
\hline APRI & $0.234 \pm 0.012$ & $0.241 \pm 0.007$ & 0.951 & $>0.85$ : advanced fibrosis \\
\hline
\end{tabular}

Data are presented as mean \pm standard error of the mean (SEM) or frequency (percentage).

*Between-groups comparison (independent sample T-test or Mann-Whitney test). *According to International Diabetes Federation (IDF) definition. ${ }^{\S}$ For patients without other cardiovascular risk factors.

ALP: alkaline phosphatase; ALT: alanine transaminase; APRI: AST to Platelet Ratio Index; AST: aspartate transaminase; BMI: body mass index; DHEAS: dehydroepiandrosterone sulfate; FAI: free androgen index; FSH: follicle-stimulating hormone; GGT: gammaglutamyl transferase; HC: hip circumference; HDL-C: high density lipoprotein cholesterol; HIS: hepatic steatosis index; HOMA-IR: homeostatic model of assessment insulin resistance; IFG: impaired fasting glucose; LAP: lipid accumulation product; LDL-C: low density lipoprotein cholesterol; LH: luteinizing hormone; MetS: metabolic syndrome; na: not applicable; NAFLD: non-alcoholic fatty liver disease; PCOS: polycystic ovary syndrome; QUICKI: quantitative insulin sensitivity check index; SHBG: sex hormone-binding protein; T2DM: type 2 diabetes mellitus; WC: waist circumference; WHR: waist to hip ratio. 
Regarding IR, insulin, HOMA-IR, HOMA- $\beta$ and frequency of MetS were significant higher, whereas QUICKI was lower in the PCOS compared with the control group (Table 2).

Regarding non-invasive indices, data for continuous ones are presented in Table 2 and Figure 1 and those for categorical (BAAT and BARD) in Table 3. All indices of hepatic steatosis (NAFLD liver fat score, LAP and HIS) were significantly higher in the PCOS than the control group (Table 2; Figure 1). Regarding hepatic fibrosis, FIB-4 and BAAT were higher in the PCOS group, whereas APRI and BARD were not (Tables 2 and 3). In any case, based on the indices of hepatic fibrosis, only a minority of PCOS women had advanced fibrosis: according to BAAT, there were only two $(0.6 \%)$ PCOS women at stage 3 and none at stage 4 , whereas, according to BARD, only three $(1 \%)$ PCOS women were at stage 4 . By applying the thresholds for advanced fibrosis (F3F4) in FIB-4 and APRI, two (0.6\%) and four (1.3\%) PCOS women, respectively, were expected to have advanced fibrosis and no women in the control group.

When patients with mild PCOS (without hyper-
Table 3. Comparative data between PCOS and control group for BAAT and BART indices

\begin{tabular}{lccc}
\hline & Control group & PCOS group & p-value* \\
\hline BAAT & & & \\
$0-1[\mathrm{~N}(\%)]$ & $74(94.9)$ & $265(84.4)$ & 0.015 \\
$2-4[\mathrm{~N}(\%)]$ & $4(5.1)$ & $49(15.6)$ & \\
BARD & & & \\
$0-1[\mathrm{~N}(\%)]$ & $37(47.4)$ & $152(48.4)$ & 0.900 \\
2-4 [N (\%)] & $41(52.6)$ & $162(51.6)$ & \\
\hline
\end{tabular}

Data are presented as frequency (percentage).

* Between-groups comparison (chi-square or Fischer exact test). BAAT: BMI Age ALT Triglycerides, BARD: BMI AST/ALT Ratio Diabetes.

androgenemia; $\mathrm{n}=31$ ) were excluded, the results were essentially unchanged for all indices. Notably, statistical significance did not change when continuous non-invasive indices were adjusted for age (Table 4). FIB-4 was not adjusted for age as the latter was included in its calculation.

Correlations between the continuous non-invasive indices and parameters related to sex hormones and IR are summarized in Table 5.
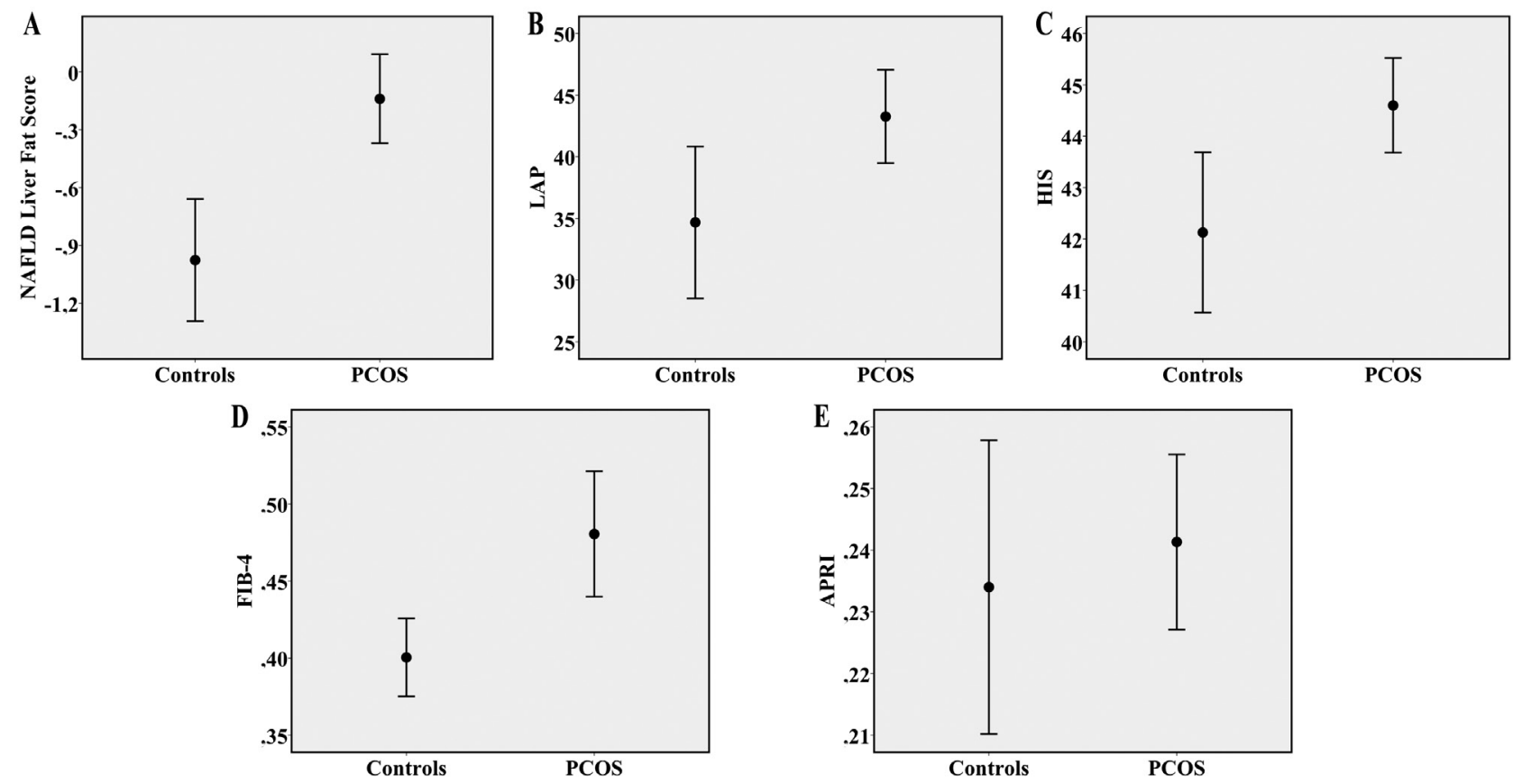

Figure 1. Error bars (mean and 95\% Confidence Interval) depicting: (A) NAFLD liver fat score; (B) LAP; (C) HIS; (D) FIB-4 and (E) APRI scores between PCOS women and controls. APRI: AST to Platelet Ratio Index; AST: aspartate transaminase; HIS: hepatic steatosis index; LAP: lipid accumulation product; NAFLD: non-alcoholic fatty liver disease. 
Table 4. Comparative data of study groups for non-invasive indices after adjustment for age

\begin{tabular}{lccc}
\hline & Control group & PCOS group & p-value* \\
\hline Women $(\mathrm{N})$ & 78 & 314 & - \\
NAFLD liver & $-0.927 \pm 0.241$ & $-0.151 \pm 0.113$ & 0.005 \\
fat score & & & \\
LAP & $30.1 \pm 4$ & $44.4 \pm 1.9$ & 0.002 \\
HIS & $41.9 \pm 1$ & $44.6 \pm 0.5$ & 0.017 \\
APRI & $0.224 \pm 0.015$ & $0.244 \pm 0.007$ & 0.260 \\
\hline
\end{tabular}

Data are estimated marginal mean \pm standard error of the mean (SEM) or frequency.

*Between-groups comparison (Analysis of covariance; ANCOVA). APRI: AST to Platelet Ratio Index; AST: aspartate transaminase; HIS: hepatic steatosis index; LAP: lipid accumulation product; NAFLD: non-alcoholic fatty liver disease; PCOS: polycystic ovary syndrome.

\section{Comparison between women with and without MetS}

Comparative data between PCOS women with $(\mathrm{n}=77)$ and without $(\mathrm{n}=237)$ MetS groups are presented in Table 6 . Age was essentially similar between groups. As expected, data regarding anthropometric, metabolic (lipid profile and uric acid), IR and sex hormone (testosterone, SHBG and FAI) parameters were more favorable in PCOS women without MetS.

Regarding the liver function tests, ALT, AST, GGT, but not ALP and AST to ALT ratio, were higher in PCOS women with MetS. All indices of hepatic steatosis (NAFLD liver fat score, LAP and HIS) were significantly higher in PCOS patients with than without MetS (Table 6). Regarding hepatic fibrosis, only BAAT was higher in PCOS patients with MetS, whereas FIB-4, APRI and BARD were similar (Tables 6 and 7). When patients with mild PCOS (without hyperandrogenemia; $n=31$ ) were excluded, the results were essentially unchanged for all indices. Notably, statistical significance did not

Table 5. Correlations between non-invasive indices and variables related to sex hormones or insulin resistance

\begin{tabular}{|c|c|c|c|c|c|}
\hline & NAFLD liver fat score & LAP & HIS & FIB-4 & APRI \\
\hline NAFLD liver fat score & - & - & - & - & - \\
\hline LAP & $0.584(<0.001)$ & - & - & - & - \\
\hline HIS & $0.594(<0.001)$ & $0.618(<0.001)$ & - & - & - \\
\hline FIB-4 & $0.252(<0.001)$ & $-0.011(0.825)$ & $0.368(<0.001)$ & - & - \\
\hline APRI & $0.060(0.236)$ & $-0.022(0.665)$ & $0.092(0.067)$ & $0.628(<0.001)$ & - \\
\hline Testosterone (nmol/L) & $0.217(<0.001)$ & $0.172(0.001)$ & $0.151(0.003)$ & $-0.156(0.002)$ & $-0.019(0.701)$ \\
\hline DHEAS ( $\mu \mathrm{mol} / \mathrm{L})$ & $0.048(0.340)$ & $0.045(0.372)$ & $0.069(0.175)$ & $-0.222(<0.001)$ & $-0.105(0.037)$ \\
\hline$\Delta 4$-androstenedione $(\mathrm{nmol} / \mathrm{L})$ & $0.085(0.094)$ & $0.041(0.413)$ & $-0.026(0.609)$ & $-0.129(0.010)$ & $0.009(0.854)$ \\
\hline SHBG (nmol/L) & $-0.481(<0.001)$ & $-0.518(<0.001)$ & $-0.431(<0.001)$ & $0.273(<0.001)$ & $0.054(0.282)$ \\
\hline FAI & $0.416(0.001)$ & $0.410(<0.001)$ & $0.350(<0.001)$ & $-0.268(<0.001)$ & $-0.053(0.298)$ \\
\hline $\mathrm{LH}(\mathrm{U} / \mathrm{L})$ & $0.039(0.441)$ & $-0.071(0.161)$ & $-0.075(0.139)$ & $-0.094(0.063)$ & $0.006(0.904)$ \\
\hline FSH (U/L) & $-0.048(0.347)$ & $-0.092(0.069)$ & $-0.027(0.596)$ & $0.040(0.432)$ & $0.122(0.016)$ \\
\hline $\mathrm{LH}$ to $\mathrm{FSH}$ ratio & $0.074(0.146)$ & $-0.014(0.782)$ & $-0.026(0.602)$ & $-0.140(0.006)$ & $-0.070(0.165)$ \\
\hline Prolactin (pmol/L) & $-0.095(0.059)$ & $-0.065(0.211)$ & $-0.104(0.039)$ & $-0.071(0.090)$ & $-0.084(0.096)$ \\
\hline Glucose $(\mathrm{mmol} / \mathrm{L})$ & $0.372(<0.001)$ & $0.342(<0.001)$ & $0.283(<0.001)$ & $0.079(0.120)$ & $0.122(0.016)$ \\
\hline Insulin (pmol/L) & $0.917(<0.001)$ & $0.557(<0.001)$ & $0.517(<0.001)$ & $0.275(<0.001)$ & $-0.067(0.186)$ \\
\hline HOMA-IR & $0.917(<0.001)$ & $0.571(<0.001)$ & $0.521(<0.001)$ & $0.268(<0.001)$ & $-0.079(0.118)$ \\
\hline НОМА- $\beta$ & $0.747(<0.001)$ & $0.407(<0.001)$ & $0.394(<0.001)$ & $0.243(<0.001)$ & $-0.010(0.845)$ \\
\hline QUICKI & $-0.917(<0.001)$ & $-0.571(<0.001)$ & $-0.521(<0.001)$ & $-0.268(<0.001)$ & $0.079(0.117)$ \\
\hline
\end{tabular}

Data are presented as Spearman's coefficient of correlation; $r_{\mathrm{s}}$ (p-value).

APRI: AST to Platelet Ratio Index; AST: aspartate transaminase; DHEAS: dehydroepiandrosterone sulfate; FAI: free androgen index; FSH: follicle-stimulating hormone; HIS: hepatic steatosis index; HOMA-IR: homeostatic model of assessment insulin resistance; LAP: lipid accumulation product; LH: luteinizing hormone; QUICKI: quantitative insulin sensitivity check index; NAFLD: non-alcoholic fatty liver disease; SHBG: sex hormone-binding protein. 
Table 6. Comparative data between PCOS with and without MetS

\begin{tabular}{|c|c|c|c|}
\hline & Non-MetS group & MetS group & p-value* \\
\hline Women [N (\%)] & $237(75.5)$ & $77(24.5)$ & - \\
\hline IFG $[\mathrm{N}(\%)]$ & $8(3.4)$ & $37(48.1)$ & $<0.001$ \\
\hline T2DM [N (\%)] & $1(0.4)$ & $5(6.5)$ & 0.004 \\
\hline Age (years) & $25.8 \pm 0.4$ & $26.8 \pm 0.8$ & 0.287 \\
\hline BMI $\left(\mathrm{kg} / \mathrm{m}^{2}\right)$ & $30.1 \pm 0.4$ & $36.8 \pm 0.8$ & $<0.001$ \\
\hline $\mathrm{WC}(\mathrm{cm})$ & $90.1 \pm 0.9$ & $104.1 \pm 1.5$ & $<0.001$ \\
\hline $\mathrm{HC}(\mathrm{cm})$ & $112.3 \pm 0.8$ & $122.7 \pm 1.4$ & $<0.001$ \\
\hline WHR & $0.804 \pm 0.005$ & $0.848 \pm 0.007$ & $<0.001$ \\
\hline Testosterone (nmol/L) & $2.48 \pm 0.07$ & $2.74 \pm 0.12$ & 0.016 \\
\hline DHEAS $(\mu \mathrm{mol} / \mathrm{L})$ & $7.98 \pm 0.24$ & $7.49 \pm 0.33$ & 0.646 \\
\hline$\Delta 4$-androstenedione $(\mathrm{nmol} / \mathrm{L})$ & $9.81 \pm 0.28$ & $9.81 \pm 0.52$ & 0.773 \\
\hline SHBG (nmol/L) & $40 \pm 1.5$ & $25.6 \pm 1.2$ & $<0.001$ \\
\hline FAI & $8.6 \pm 0.5$ & $13 \pm 1$ & $<0.001$ \\
\hline Prolactin (pmol/L) & $587 \pm 17$ & $517 \pm 26$ & 0.079 \\
\hline $\mathrm{LH}(\mathrm{U} / \mathrm{L})$ & $7.8 \pm 0.3$ & $7.3 \pm 0.6$ & 0.428 \\
\hline FSH (U/L) & $5.9 \pm 0.1$ & $5.5 \pm 0.2$ & 0.112 \\
\hline LH to $\mathrm{FSH}$ ratio & $1.38 \pm 0.06$ & $1.37 \pm 0.10$ & 0.951 \\
\hline $\operatorname{AST}(\mathrm{U} / \mathrm{L})$ & $19.2 \pm 0.5$ & $22.2 \pm 1.2$ & 0.020 \\
\hline $\operatorname{ALT}(\mathrm{U} / \mathrm{L})$ & $25.8 \pm 1$ & $30.2 \pm 2.4$ & 0.046 \\
\hline AST to ALT ratio & $0.903 \pm 0.031$ & $0.859 \pm 0.041$ & 0.461 \\
\hline GGT (U/L) & $19.7 \pm 0.8(N=125)$ & $26.5 \pm 3(N=19)$ & 0.041 \\
\hline $\operatorname{ALP}(\mathrm{U} / \mathrm{L})$ & $76.8 \pm 1.4$ & $76.9 \pm 2$ & 0.981 \\
\hline Total cholesterol (mmol/L) & $4.92 \pm 0.06$ & $5.19 \pm 0.13$ & 0.046 \\
\hline HDL-C (mmol/L) & $1.38 \pm 0.02$ & $1 \pm 0.02$ & $<0.001$ \\
\hline LDL-C (mmol/L) & $3.08 \pm 0.06$ & $3.40 \pm 0.11$ & 0.012 \\
\hline Triglycerides (mmol/L) & $0.94 \pm 0.02$ & $1.63 \pm 0.08$ & $<0.001$ \\
\hline Uric acid $(\mu \mathrm{mol} / \mathrm{L})$ & $268 \pm 6$ & $321 \pm 6$ & $<0.001$ \\
\hline Platelets $\left(\mathrm{N} x 10^{3} / \mu \mathrm{L}\right)$ & $275 \pm 4$ & $304 \pm 8$ & 0.001 \\
\hline Glucose (mmol/L) & $5.32 \pm 0.03$ & $5.98 \pm 0.07$ & $<0.001$ \\
\hline Insulin (pmol/L) & $100.7 \pm 4.2$ & $161.1 \pm 11.8$ & $<0.001$ \\
\hline HOMA-IR & $3.48 \pm 0.14$ & $6.22 \pm 0.44$ & $<0.001$ \\
\hline HOMA- $\beta$ & $173 \pm 10$ & $196 \pm 14$ & 0.012 \\
\hline QUICKI & $0.328 \pm 0.002$ & $0.302 \pm 0.003$ & $<0.001$ \\
\hline NAFLD liver fat score & $-0.793 \pm 0.099$ & $1.874 \pm 0.258$ & $<0.001$ \\
\hline LAP & $33.4 \pm 1.4$ & $76.8 \pm 4.9$ & $<0.001$ \\
\hline HIS & $43 \pm 0.5$ & $49.8 \pm 1$ & $<0.001$ \\
\hline FIB-4 & $0.400 \pm 0.015$ & $0.401 \pm 0.024$ & 0.984 \\
\hline APRI & $0.239 \pm 0.008$ & $0.252 \pm 0.016$ & 0.881 \\
\hline
\end{tabular}

Data are presented as mean \pm standard error of the mean (SEM) or frequency (percentage).

*Between-groups comparison (independent sample T-test or Mann-Whitney test).

\#According to International Diabetes Federation (IDF) definition.

ALP: alkaline phosphatase; ALT: alanine transaminase; AST: aspartate transaminase; BMI: body mass index; DHEAS: dehydroepiandrosterone sulfate; FAI: free androgen index; FSH: follicle-stimulating hormone; GGT: gamma-glutamyl transferase; HC: hip circumference; HDL-C: high density lipoprotein cholesterol; HIS: hepatic steatosis index; HOMA-IR: homeostatic model of assessment insulin resistance; IFG: impaired fasting glucose; LAP: lipid accumulation product; LDL-C: low density lipoprotein cholesterol; LH: luteinizing hormone; MetS: metabolic syndrome; QUICKI: quantitative insulin sensitivity check index; NAFLD: non-alcoholic fatty liver disease; PCOS: polycystic ovary syndrome; SHBG: sex hormone-binding protein; T2DM: type 2 diabetes mellitus; WC: waist circumference; WHR: waist to hip ratio. 

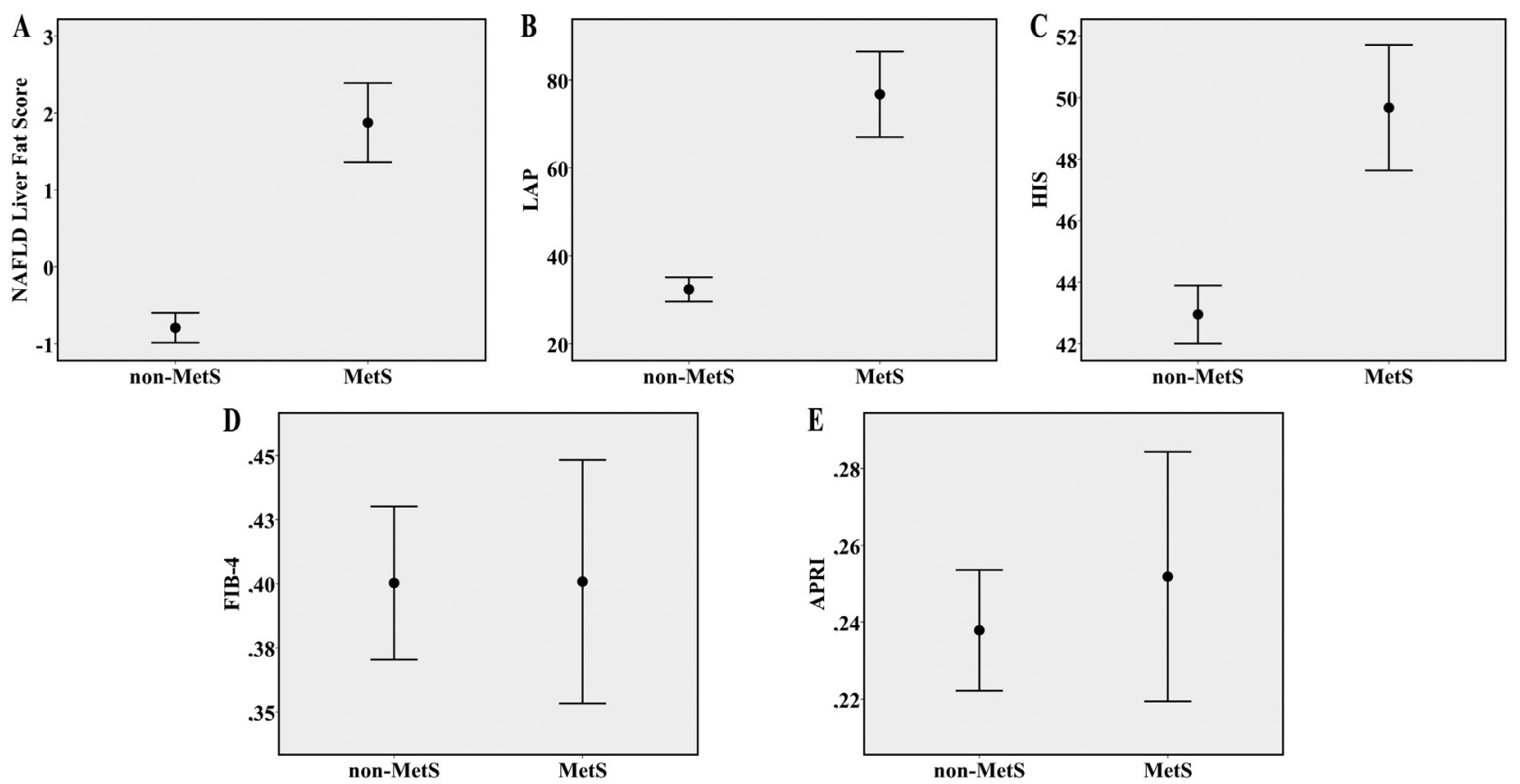

Figure 2. Error bars (mean and 95\% Confidence Interval) depicting: (A) NAFLD liver fat score; (B) LAP; (C) HIS; (D) FIB-4 and (E) APRI scores between PCOS patients with and without MetS. APRI: AST to Platelet Ratio Index; AST: aspartate transaminase; HIS: hepatic steatosis index; LAP: lipid accumulation product; MetS: metabolic syndrome; NAFLD: non-alcoholic fatty liver disease.

Table 7. Comparative data between PCOS women with and without MetS group for BAAT and BART indices

\begin{tabular}{lccc}
\hline & Non-MetS group & MetS group & p-value* \\
\hline BAAT & & & \\
$0-1[\mathrm{~N}(\%)]$ & $227(95.8)$ & $38(49.4)$ & $<0.001$ \\
2-4 [N (\%)] & $10(4.2)$ & $39(50.6)$ & \\
BARD & & & \\
0-1 [N (\%)] & $113(47.7)$ & $39(50.6)$ & 0.650 \\
2-4 [N (\%)] & $124(50.6)$ & $38(49.4)$ & \\
\hline
\end{tabular}

Data are presented as frequency (percentage)

*Between-groups comparison (chi-square or Fischer exact test) BAAT: BMI Age ALT Triglycerides; BARD: BMI AST/ALT Ratio Diabetes; MetS: metabolic syndrome.

change when continuous non-invasive indices were adjusted for age (data not shown).

Similarly, when the comparison was performed among four groups [control/non-MetS $(n=68)$ vs. control/MetS $(n=10)$ vs. PCOS/non-Mets (237) vs. PCOS/MetS $(n=77)]$, all indices of hepatic steatosis showed a trend towards higher values when moving towards PCOS and MetS [NAFLD liver fat score: $p<0.001$; LAP: $p<0.001$; HIS: $p<0.001]$.

\section{DISCUSSION}

To our knowledge, this is the first study reporting data on combined non-invasive indices of hepatic steatosis and fibrosis in women with PCOS, which are of relatively low cost and can be calculated on a routine basis. Indices of hepatic steatosis (NAFLD liver fat score, LAP and HIS) were significantly higher in the PCOS than the control group, as well as in PCOS women with than without MetS. However, regarding indices of hepatic fibrosis, FIB-4 and BAAT, but not APRI and BARD, were higher in the PCOS than the control group. In PCOS women with MetS, only BAAT was higher compared with PCOS women without MetS. When non-invasive indices were adjusted for age, the statistical significance remained essentially unchanged for all comparisons.

The results of this series seem to be clear for indices of hepatic steatosis, but not for those of fibrosis. This discordance in fibrosis indices may be partly attributed to the low rates of hepatic fibrosis expected in this cohort of relatively young women $(26.1 \pm 0.4$ years) with a low rate of T2DM $(1.9 \%)$ and relatively low concentrations of triglycerides 
$(1.11 \pm 0.03 \mathrm{mmol} / \mathrm{L})$. As shown, only a minority of women with PCOS in this cohort were expected to have advanced fibrosis according to the thresholds of all fibrosis indices. However, the high rates of hepatic steatosis, together with higher IR (HOMAIR, HOMA- $\beta$, QUICKI) and obesity (BMI, WC) in their third decade, may possibly render PCOS women at higher risk for hepatic inflammation and fibrosis later in their life, given that approximately $15-30 \%$ of patients with non-alcoholic simple steatosis evolve to non-alcoholic steatohepatitis (NASH). ${ }^{6}$ Simple steatosis consists mainly of steatosis, whereas NASH in addition to steatosis features progressive inflammation and fibrosis; ${ }^{6}$ this distinction is of importance, since simple steatosis progresses to cirrhosis in less than $5 \%$ of cases, whereas NASH progresses to cirrhosis in $10-15 \%$ of cases over 10 years and in $25-30 \%$ of cases in the presence of advanced fibrosis. ${ }^{5,21}$ Furthermore, NASH, but not simple steatosis, predisposes to hepatocellular carcinoma. ${ }^{5,21}$

Regarding the indices of hepatic steatosis, similarly to our findings other studies have reported higher rates of ultrasound-proven hepatic steatosis in PCOS $(31-73 \%)$ than non-PCOS controls (18-47\%). ${ }^{22-25}$ Steatosis was also shown to be positively associated with HOMA-IR ${ }^{23,26}$ and $\mathrm{FAI}^{23}$ but negatively with SHBG ${ }^{23}$ as in our study. Hepatic fat, quantified by proton-magnetic resonance spectroscopy, was also found to be higher in women with PCOS $(n=29)$ than in controls $(\mathrm{n}=22) .{ }^{27}$ Interestingly, hyperandrogenic women with PCOS $(n=19)$ had higher IR and hepatic fat than non-hyperandrogenic ones $(n=10) .{ }^{27}$ Likewise, in our study, women with PCOS with MetS had higher IR, higher testosterone concentrations and FAI and higher rates of indices for hepatic steatosis compared with PCOS women without MetS.

Contrary to our findings and existing literature, there is one study of 17 lean women with PCOS (with normal aminotransferases) and 17 controls in whom hepatic steatosis was not detected (by ultrasound and computed tomography) in any woman. ${ }^{28}$ The reasons for this inconsistency are not well understood, but the selection bias (lean women with normal liver function tests) may be a plausible cause. However, although obesity certainly predisposes to NAFLD,${ }^{8}$ high rates of NAFLD (approximately 40\%) have been reported even in lean and young PCOS women. ${ }^{26,29}$
Data regarding hepatic fibrosis in women with PCOS are rather scarce because this requires liver biopsy which has to date been performed only on a very limited number of patients in relevant studies. In one study, six (of a total of 200 retrospectively evaluated) women with PCOS and persistent aminotransferase elevation underwent liver biopsy: all had NASH with fibrosis (3\%). ${ }^{30}$ This low fibrosis rate is in accordance with our findings, based on non-invasive fibrosis indices. By contrast, in another study partly based on liver biopsy, women with PCOS $(\mathrm{n}=34 ; 25$ subjected to liver biopsy; 11 with NASH) had a non-statistical trend towards higher rates for NASH than non-PCOS controls ( $n=32 ; 25$ subjected to liver biopsy; 5 with NASH). ${ }^{31}$ Different rates of $\mathrm{NASH}$ between these studies may possibly reflect population differences and/or selection bias; further, large-scale studies are needed to clarify the prevalence of NASH in women with PCOS. However, irrespective of the rate of NASH, some women with PCOS have advanced fibrosis, even early in their lives, and they may benefit if NASH is diagnosed. Persistent aminotransferase elevation alone may provide an indication, but combined non-invasive indices (some of which contain AST and/or ALT) are currently regarded as more sensitive tools to select these patients for liver biopsy. ${ }^{11}$

Data evaluating the reverse issue, being the rates of PCOS in NAFLD populations, are currently rather limited. In one study, 10 of 14 (71\%) premenopausal women with NAFLD (biopsy-proven in 7, ultrasoundproven in 7) were reported to have PCOS. ${ }^{32}$ In another study, patients with biopsy-proven simple steatosis and PCOS $(\mathrm{n}=12)$ had higher serum cytokeratin-18 (M30) compared with those with simple steatosis but without PCOS $(n=12)$, despite similar serum liver function tests, serum lipids and IR. ${ }^{31}$ However, the small sample size of both studies possibly resulted in low statistical power.

Indices of hepatic steatosis correlated with each other (Table 5). FIB-4 also correlated with APRI, NAFLD liver fat score and HIS, but not LAP. APRI did not correlate with indices of hepatic steatosis. All indices of hepatic steatosis correlated with insulin and IR in this study; FIB-4 also correlated with insulin and IR, although less strongly. This was expected given that IR contributes to the pathogenesis of hepatic 
steatosis, but also, possibly to a lower degree, to the progression from the hepatic steatosis to NASH, which usually features fibrosis. ${ }^{8}$ Furthermore, all indices of hepatic steatosis correlated positively with testosterone and FAI but inversely with SHBG. Interestingly, testosterone, DHEAS, $\Delta_{4}$-androstenedione and FAI inversely correlated with FIB-4. Likewise, higher concentrations of calculated free testosterone, bioavailable testosterone and FAI, whereas lower concentrations of SHBG, were shown in postmenopausal women with NAFLD than age- and BMI-matched controls. ${ }^{33}$ Although correlations cannot prove a cause-effect association, it could be speculated that hyperandogenemia, which is usually observed in women with PCOS, may play a permissive role in the pathogenesis of hepatic steatosis but a potential protective role in the progression to NASH. This hypothesis is in agreement with low fibrosis rates in PCOS women observed in this study, but requires validation from studies of different design.

This study has certain strengths and limitations. It is a relatively large-scale study and the first based on seven different non-invasive indices of hepatic steatosis and fibrosis. On the other hand, the limitations of the study are the following. 1) Data were retrospectively reviewed for the need of this study; however, they had been previously prospectively and systematically recorded for the need of other studies. 2) The participants were not subjected to liver biopsy, regarded as the diagnostic gold standard $;{ }^{34}$ however, the performance of liver biopsy in young women with low or no burden of metabolic co-morbidity (i.e., T2DM, dyslipidemia) and low hepatic fibrosis risk raises obvious ethical considerations. 3) Other liver diseases were excluded on the basis of history and self-reporting and no specific tests were used for this aim; however, the rates of other liver diseases in this cohort of young women are expected to be very low. 4) The control group were of higher age than the PCOS group; however, although the prevalence of NAFLD increases with increasing age,${ }^{5}$ women with PCOS had higher non-invasive indices, especially those related to hepatic steatosis, but also to FIB-4 and BAAT (which also increase with increasing age), even if they were of lower age than controls. In any case, adjustment for age did not change the results. 5) The control group had lower BMI and WC than the PCOS group; however, most of the presenting non-invasive indices (with the exemption of FIB-4 and APRI) include a variable of adiposity (i.e., BMI, WC or metabolic syndrome), which implies a type of internal adjustment. 6) Data for GGT should be cautiously interpreted because they were available only for a subgroup of participants $(66.7 \%$ of the control and $45.9 \%$ of the PCOS group, respectively). 7) Other non-invasive indices of hepatic steatosis or fibrosis [i.e., fatty liver index (FLI), NAFLD fibrosis score, etc] were not calculated because at least one of the required parameters for their estimation was not available or was available only for a subset of the participants. 8) Liver ultrasound was not performed in this study, mainly due to its retrospective nature; however, the sensitivity (60-94\%) and specificity (66$100 \%$ ) of liver ultrasound varies, being lower in obesity, ${ }^{35}$ which usually co-exists with PCOS and NAFLD. In addition, it has low inter- and intra-observer variability and, most importantly, the ultrasound cannot provide information about hepatic fibrosis. ${ }^{35}$

In conclusion, indices of hepatic steatosis (NAFLD liver fat score, LAP and HIS) were all significantly higher in the PCOS than the control group, as well as in PCOS women with rather than without MetS in this study, indicating a common link, especially in the presence of MetS. Regarding indices of hepatic fibrosis, controversial results were retrieved, possibly because of the expected low rates of hepatic fibrosis in this series of young women with low relevant comorbidity. However, despite the low rate of hepatic fibrosis, the selection of these PCOS women with high NASH probability would be of paramount importance, since these women are expected to benefit the most from histological validation and subsequent lifestyle and pharmacological interventions. Further studies are warranted to validate hepatic steatosis and fibrosis indices in PCOS populations and to evaluate the long-term outcomes of these indices in PCOS women.

\section{FUNDING}

This study did not receive any funding.

\section{DISCLOSURE STATEMENT}

There is no conflict of interest related to this manuscript. 


\section{REFERENCES}

1. Panidis D, Tziomalos K, Papadakis E, Vosnakis C, Chatzis P, Katsikis I, 2013 Lifestyle intervention and anti-obesity therapies in the polycystic ovary syndrome: impact on metabolism and fertility. Endocrine 44: 583590.

2. Rotterdam ESHRE/ASRM-Sponsored PCOS consensus workshop group, 2004 Revised 2003 consensus on diagnostic criteria and long-term health risks related to polycystic ovary syndrome (PCOS). Hum Reprod 19: 41-47.

3. Carmina E, 2012 PCOS: metabolic impact and longterm management. Minerva Ginecol 64: 501-505.

4. Sanyal AJ, 2011 NASH: A global health problem. Hepatol Res 41: 670-674.

5. Vernon G, Baranova A, Younossi ZM, 2011 Systematic review: the epidemiology and natural history of non-alcoholic fatty liver disease and non-alcoholic steatohepatitis in adults. Aliment Pharmacol Ther 34: 274-285.

6. Polyzos SA, Kountouras J, Zavos C, Deretzi G, 2012 Nonalcoholic fatty liver disease: multimodal treatment options for a pathogenetically multiple-hit disease. J Clin Gastroenterol 46: 272-284.

7. Panidis D, Macut D, Tziomalos K, et al, 2013 Prevalence of metabolic syndrome in women with polycystic ovary syndrome. Clin Endocrinol (Oxf) 78: 586-592.

8. Polyzos SA, Kountouras J, Zavos C, 2009 Nonalcoholic fatty liver disease: the pathogenetic roles of insulin resistance and adipocytokines. Curr Mol Med 9: 299314.

9. Baranova A, Tran TP, Birerdinc A, Younossi ZM, 2011 Systematic review: association of polycystic ovary syndrome with metabolic syndrome and non-alcoholic fatty liver disease. Aliment Pharmacol Ther 33: 801-814.

10. Chalasani N, Younossi Z, Lavine JE, et al, 2012 The diagnosis and management of non-alcoholic fatty liver disease: practice Guideline by the American Association for the study of liver diseases, American College of Gastroenterology, and the American Gastroenterological Association. Hepatology 55: 2005-2023.

11. Machado MV, Cortez-Pinto H, 2013 Non-invasive diagnosis of non-alcoholic fatty liver disease. A critical appraisal. J Hepatol 58: 1007-1019.

12. Panidis D, Tziomalos K, Misichronis G, et al, 2012 Insulin resistance and endocrine characteristics of the different phenotypes of polycystic ovary syndrome: a prospective study. Hum Reprod 27: 541-549.

13. Piouka A, Farmakiotis D, Katsikis I, Macut D, Gerou S, Panidis D, 2009 Anti-Mullerian hormone levels reflect severity of PCOS but are negatively influenced by obesity: relationship with increased luteinizing hormone levels. Am J Physiol Endocrinol Metab 296: E238-243.

14. Kotronen A, Peltonen M, Hakkarainen A, et al, 2009 Prediction of non-alcoholic fatty liver disease and liver fat using metabolic and genetic factors. Gastroenterology 137: 865-872.

15. Bedogni G, Kahn HS, Bellentani S, Tiribelli C, 2010 A simple index of lipid overaccumulation is a good marker of liver steatosis. BMC Gastroenterol 10: 98.

16. Lee JH, Kim D, Kim HJ, et al, 2010 Hepatic steatosis index: a simple screening tool reflecting nonalcoholic fatty liver disease. Dig Liver Dis 42: 503-508.

17. McPherson S, Stewart SF, Henderson E, Burt AD, Day CP, 2010 Simple non-invasive fibrosis scoring systems can reliably exclude advanced fibrosis in patients with non-alcoholic fatty liver disease. Gut 59: 1265-1269.

18. Kruger FC, Daniels CR, Kidd M, et al, 2011 APRI: a simple bedside marker for advanced fibrosis that can avoid liver biopsy in patients with NAFLD/NASH. S Afr Med J 101: 477-480.

19. Ratziu V, Giral P, Charlotte F, et al, 2000 Liver fibrosis in overweight patients. Gastroenterology 118: 1117-1123.

20. Harrison SA, Oliver D, Arnold HL, Gogia S, Neuschwander-Tetri BA, 2008 Development and validation of a simple NAFLD clinical scoring system for identifying patients without advanced disease. Gut 57: 1441-1447.

21. Musso G, Gambino R, Cassader M, Pagano G, 2011 Meta-analysis: natural history of non-alcoholic fatty liver disease (NAFLD) and diagnostic accuracy of non-invasive tests for liver disease severity. Ann Med 43: 617-649.

22. Cerda C, Perez-Ayuso RM, Riquelme A, et al, 2007 Nonalcoholic fatty liver disease in women with polycystic ovary syndrome. J Hepatol 47: 412-417.

23. Vassilatou E, Lafoyianni S, Vryonidou A, et al, 2010 Increased androgen bioavailability is associated with non-alcoholic fatty liver disease in women with polycystic ovary syndrome. Hum Reprod 25: 212-220.

24. Qu ZY, Shi YH, Zhao DN, Jiang JJ, Ma ZX, Chen ZJ, 2010 Effect of obesity on nonalcoholic fatty liver disease in Chinese women with polycystic ovary syndrome. Zhonghua Yi Xue Za Zhi 90: 2036-2039.

25. Zueff LF, Martins WP, Vieira CS, Ferriani RA, 2012 Ultrasonographic and laboratory markers of metabolic and cardiovascular disease risk in obese women with polycystic ovary syndrome. Ultrasound Obstet Gynecol 39: 341-347.

26. Gambarin-Gelwan M, Kinkhabwala SV, Schiano TD, Bodian C, Yeh HC, Futterweit W, 2007 Prevalence of nonalcoholic fatty liver disease in women with polycystic ovary syndrome. Clin Gastroenterol Hepatol 5: 496-501.

27. Jones H, Sprung VS, Pugh CJ, et al, 2012 Polycystic ovary syndrome with hyperandrogenism is characterized by an increased risk of hepatic steatosis compared to nonhyperandrogenic PCOS phenotypes and healthy controls, independent of obesity and insulin resistance. J Clin Endocrinol Metab 97: 3709-3716.

28. Markou A, Androulakis, II, Mourmouris C, et al, 2010 
Hepatic steatosis in young lean insulin resistant women with polycystic ovary syndrome. Fertil Steril 93: 12201226.

29. Ciotta L, Pagano I, Stracquadanio M, Formuso C, 2011 Polycystic ovarian syndrome incidence in young women with non-alcoholic fatty liver disease. Minerva Ginecol 63: 429-437.

30. Setji TL, Holland ND, Sanders LL, Pereira KC, Diehl AM, Brown AJ, 2006 Nonalcoholic steatohepatitis and nonalcoholic Fatty liver disease in young women with polycystic ovary syndrome. J Clin Endocrinol Metab 91: 1741-1747.

31. Baranova A, Tran TP, Afendy A, et al, 2013 Molecular signature of adipose tissue in patients with both nonalcoholic fatty liver disease (NAFLD) and polycystic ovarian syndrome (PCOS). J Transl Med 11: 133.
32. Brzozowska MM, Ostapowicz G, Weltman MD, 2009 An association between non-alcoholic fatty liver disease and polycystic ovarian syndrome. J Gastroenterol Hepatol 24: 243-247.

33. Polyzos SA, Kountouras J, Tsatsoulis A, et al, 2013 Sex steroids and sex hormone-binding globulin in postmenopausal women with non-alcoholic fatty liver disease. Hormones (Athens) 12: 405-416.

34. Sanyal AJ, Brunt EM, Kleiner DE, et al, 2011 Endpoints and clinical trial design for nonalcoholic steatohepatitis. Hepatology 54: 344-353.

35. Schwenzer NF, Springer F, Schraml C, Stefan N, Machann J, Schick F, 2009 Non-invasive assessment and quantification of liver steatosis by ultrasound, computed tomography and magnetic resonance. J Hepatol 51: 433-445. 


\title{
Short-chain fatty acids increase expression and secretion of stromal cell-derived factor-1 in mouse and human pre-adipocytes
}

\author{
Venkat N. Vangaveti, ${ }^{1}$ Catherine Rush, ${ }^{2}$ Linda Thomas, ${ }^{1}$ Roy R. Rasalam, ${ }^{1}$ \\ Usman H. Malabu, ${ }^{1}$ Scott G. McCoombe, ${ }^{3}$ Richard L. Kennedy ${ }^{3}$ \\ ${ }^{1}$ School of Medicine and Dentistry, ${ }^{2}$ School of Veterinary and Biomedical Sciences, James Cook University, Queensland; \\ ${ }^{3}$ School of Medicine, Deakin University, Victoria; Australia
}

\begin{abstract}
OBJECTIVE: Stromal cell-derived factor-1 (SDF-1) is expressed in pre-adipocytes but its role is unknown. We investigated butyrate (a histone deacetylase inhibitor - HDACi) and other short-chain fatty acids (SCFA) in the regulation of SDF-1. We further investigated whether effects of SCFA were signalled through G protein-coupled receptors FFA2 and FFA3. DESIGN AND RESULTS: SDF-1 mRNA expression and protein secretion were studied in 3T3-L1 cells and human pre-adipocytes. SDF-1 was abundant, with mRNA and protein levels increased by butyrate. This was replicated with acetate and propionate, but not with trichostatin or valproate. Trichostatin inhibited SDF-1 secretion. Pertussis toxin blocked stimulation by butyrate. The order of potency of SCFA in stimulating SDF-1 (C3 > C4 > C2) is consistent with action through FFA3. Silencing the FFA3 gene abolished butyrate-stimulated SDF-1 expression and secretion. FFA3 was expressed in both pre-adipocytes and adipocytes, while FFA2 was expressed in adipocytes only. SDF-1 expression was low in murine macrophage J774.2 cells, while the SDF-1 receptor CXCR4 was absent from 3T3-L1 cells but abundant in J774.2 macrophages. In human pre-adipocytes, FFA3 was also expressed and SCFA increased SDF-1 secretion. CONCLUSIONS: SDF-1 and CXCR4 may mediate the interaction between adipose stromal cells and macrophages. Effects of SCFA are mediated through FFA3, but not histone deacetylase inhibition.
\end{abstract}

Key words: Butyrate, Fatty acids, FFA2, FFA3, Pre-adipocytes, Propionate

Address for correspondence:

Professor R.L. Kennedy, School of Medicine,

Deakin University, Waurn Ponds Campus, Geelong VIC 3220,

Australia, Tel.: 03-5227-1275, Fax: 03-5246-2986,

E-mail: richardlee.k@hotmail.com

Received 23-12-2013, Accepted 03-04-2014

\section{INTRODUCTION}

Interest in the application of fatty acids as signalling molecules has increasingly grown since the recognition of a series of $\mathrm{G}$ protein-coupled receptors with fatty acids as their ligands. ${ }^{1,2}$ Different receptors are known 
to have specificities for fatty acids of differing chain length. ${ }^{1}$ Department of 7TMR Assay Development and Compound Profiling (ADCP FFA2 (GPR43) and FFA3 (GPR41) are receptors for short-chain fatty acids (SCFA) and represent a crucial part of the body's nutrient-sensing apparatus. ${ }^{2}$ Activation of FFA2 has been reported to increase incretin secretion by enteroendocrine cells, to enhance adipogenesis, to increase leptin secretion by adipocytes, and to inhibit lipolysis..$^{3-6}$ It is well-established that FFA2 is highly expressed in adipose tissue ${ }^{4}$ but may not be involved in the pathogenesis of human obesity. ${ }^{7}$ The role and expression of FFA3 is less clearly defined. Debate continues over FFA3 expression in adipose tissue, with some groups reporting no expression, ${ }^{3,5}$ while others report robust FFA3 expression in both adipose tissue and in differentiated Ob-luc adipocytes. ${ }^{8}$ Xiong et $\mathrm{al}^{8}$ also show that SCFA, acting through FFA3, increases leptin secretion and inhibits lipolysis. Taken together, these studies indicate considerable uncertainty as to the relative roles of FFA2 and FFA3 in the pathogenesis of obesity and its complications.

Interaction between cells of adipocyte lineage and those derived from bone marrow is critical in the angiogenesis accompanying adipose tissue remodelling. ${ }^{9}$ It is also important in the low-grade inflammation associated with obesity, ${ }^{10,11}$ which predisposes to insulin resistance and atherogenesis. ${ }^{12,13}$ These processes are known to be influenced by SCFA, produced in the gut by fermentation of complex carbohydrates. ${ }^{14-16}$ Stromal cell-derived factor-1 (SDF-1, CXCL12) is a chemokine which, through interaction with its obligate receptor CXCR4, is involved in angiogenesis, stem cell trafficking, and tumour metastasis. ${ }^{17-19}$ The role of the SDF-1/CXCR4 axis in adipose tissue biology has not been extensively studied to date. Interestingly, SDF-1 expression in 3T3-L1 adipocytes has been reported in two genomic studies ${ }^{20,21}$ and one proteomic study. ${ }^{22}$ Furthermore, expression of SDF-1 has been noted in adipocyte precursors with expression diminishing during adipogenesis, ${ }^{23}$ and more recently has been shown to interact with complement-derived factors in regulating adipocyte development. ${ }^{24}$ Modulation of the SDF-1/CXCR 4 axis by butyrate has long been known to modulate growth of tumour tissues. ${ }^{25,26}$

The aim of this work was to examine if SCFA could affect SDF-1 gene expression and protein secretion in pre-adipocytes and investigate the mechanisms underlying this effect. More specifically, we sought to determine the relative roles of the two SFCA receptors, FFA2 and FFA3, and the role of histone deacetylase inhibition in regulating SDF-1 expression using the murine 3T3-L1 line and primary cultures of human pre-adipocytes.

\section{MATERIALS AND METHODS}

\section{Culture and differentiation of murine 3T3-L1 cells}

3T3-L1 cells (Sigma Aldrich Pty Ltd, NSW, Australia) were seeded (approximately 20,000/well) in 24-well plates and grown to confluence in Dulbecco's Modified Eagle Medium containing $25 \mathrm{mM}$ glucose, bovine serum, $4 \mathrm{mM}$ L-glutamine, $100 \mathrm{U} / \mathrm{mL}$ penicillin, and $100 \mu \mathrm{g} / \mathrm{mL}$ streptomycin (DMEM) supplemented with $10 \%$ foetal bovine serum (FBS) at $37^{\circ} \mathrm{C}$ in a $5 \% \mathrm{CO}_{2}$ atmosphere. For experiments on undifferentiated cells, supplement was continued for three days after reaching cell culture confluence and then replaced with DMEM containing 10\% FBS without antibiotics for 24 hours before treatment with valproate or trichostatin. For experiments on differentiated cells, once pre-adipocytes were confluent, the medium was replaced with DMEM containing $10 \%$ FBS plus penicillin and streptomycin for three days. Differentiation was then initiated using $0.5 \mathrm{mM}$ IBMX, $1 \mu \mathrm{M}$ dexamethasone, and $10 \mu \mathrm{g} / \mathrm{ml}$ insulin in high glucose DMEM supplemented with $10 \%$ FBS containing antibiotics (IBMX medium) for 48 hours. The cells were then maintained in DMEM supplemented with $10 \%$ FBS, insulin, and antibiotics until fully differentiated (typically 8-10 days). Prior to treatment with trichostatin or valproate, the cells were supplemented with DMEM medium with $10 \%$ FBS without antibiotics for 24 hours.

\section{Culture of human pre-adipocytes}

Approvals for the use of human tissues in this study were obtained from Queensland Health and James Cook University Human Research Ethics Committees. Adipose tissue was obtained from consenting adult patients following elective liposuction. $6 \mathrm{ml}$ of adipose tissue was digested with $0.1 \%$ collagenase in PBS/ BSA and placed in an orbital shaker for 60 minutes at $37^{\circ} \mathrm{C}$. The solution was then filtered through a mesh $(63 \mu \mathrm{m})$ into a $50 \mathrm{~mL}$ Falcon tube and centrifuged at 
$200 \mathrm{~g}$ for 10 minutes. With the supernatant discarded, the re-suspended pellet was treated with erythrocyte lysing buffer, incubated for 5 minutes at $37^{\circ} \mathrm{C}$ and centrifuged at $200 \mathrm{~g}$ for 5 minutes. Cells were counted in inoculation medium (basal medium: $\operatorname{DMEM}(50 \%)$, Ham's F-12 (50\%), HEPES, $\mathrm{NaHCO}_{3}$, biotin, and pantothenate supplemented with $10 \%$ FBS and $0.5 \%$ gentamicin) and seeded at a density of 150,000 cells per $\mathrm{ml}$. After 16 hours culture, the cells were washed with Dulbecco's Phosphate Buffered Saline (DPBS) and replaced with stimulation medium (basal medium with human transferrin, insulin, cortisol and fibroblast growth factor) until confluence was reached. To obtain adipocytes, the cells were incubated with adipogenic medium (basal medium with transferrin, gentamicin, triiodothyronine, insulin, and hydrocortisone). The medium was changed regularly until the cells differentiated. The cells were then stained for oil droplets.

\section{Experiments on confluent 3T3-L1 pre- adipocytes and differentiated adipocytes}

Treatments were prepared in DMEM supplemented with $10 \%$ FBS. Cells were washed with DPBS prior to addition of each treatment. $1 \mathrm{ml}$ of treatment medium was added to each well and cells incubated at $37^{\circ} \mathrm{C}, 5 \% \mathrm{CO}_{2}$ for the appropriate time period. Cell medium was collected and stored at $-80^{\circ} \mathrm{C}$ for protein expression studies. Cells were washed with DPBS $(2 \times 1 \mathrm{ml})$ and $200 \mu \mathrm{l}$ of trypsin solution added to each well. After 5 minutes incubation, the trypsin solution was neutralized with $1 \mathrm{ml}$ of DMEM containing $10 \%$ FBS. The solution containing the cells was centrifuged at $460 \mathrm{~g}$ for 5 minutes at $21^{\circ} \mathrm{C}$. Medium was removed and cells were stored at $-80^{\circ} \mathrm{C}$ pending RNA extraction.

\section{Oil red $O$ staining and FFA3 immunocytochemistry}

Staining of accumulated lipid was performed according to kit instructions. Briefly, medium was removed from cells that were then washed with DPBS. $0.5 \mathrm{ml}$ Oil red O staining solution (Chemicon Australia Pty Ltd, Vic, Australia) was added to cells cultured in a 24-well plate and incubated for 15 minutes at room temperature. Staining solution was removed and cells washed with wash solution. Immunocytochemistry was used to evaluate FFA3 and FFA2 expression on THP-1 monocytes and human cultured pre-adipocytes.
This was performed using rabbit polyclonal antibodies to FFA3 and FFA2 (Sapphire Biosciences, Redfern, NSW, Australia). THP-1 cells: $1 \times 10^{5}$ monocytes in $200 \mu \mathrm{L}$ medium were cytospun onto sialinised coverslips (Cytospin 4 centrifuge, Thermo Fischer Scientific, Scoresby, Vic, Australia) for 5 minutes at 500rpm. Coverslips were then air-dried and fixed in $75 \%$ ethanol and DPBS for 10 minutes.

Human pre-adipocytes cultured on slides were rehydrated with DPBS and incubated with $0.5 \%$ $\mathrm{H}_{2} \mathrm{O}_{2}$ to quench endogenous peroxidase activity for 10 minutes, then washed with Tris-buffered Saline with $0.05 \%$ Tween-20 (TBST buffer). This was followed by Tris Neutral Buffer (TNB) for 30 minutes and subsequently by rabbit polyclonal FFA3 antibody or isotype control (45 minutes), biotinylated rabbit IgG-G (30 minutes), HRP-streptavidin (30 minutes) with TBST wash between each step. We then added 3,3'-diaminobenzidine (DAB) chromogen prior to dehydration using an alcohol and xylene series, after staining the nucleus with Mayer's haematoxylin. Digital images were obtained using an Olympus CKX41 microscope.

\section{Small interfering RNA (siRNA) knockdown of FFA3}

3T3-L1 pre-adipocytes were plated (20,000 cells/ well) on a 6-well plate in high glucose DMEM, containing L-glutamine, penicillin, streptomycin, and supplemented with $10 \%$ FBS. The cells were incubated at $37^{\circ} \mathrm{C}$ in a $5 \% \mathrm{CO}_{2}$ atmosphere and grown to $80 \%$ confluence. The medium was replaced with DMEM containing glutamine but without serum and antibiotics $(2 \mathrm{ml} /$ well). The siRNA complex was formed with four separate double-stranded siRNAs designed to silence FFA3 (Qiagen Pty Ltd, Vic, Australia):

CAGAGTGCCAGTTGTCCAATA, CAGCCTGGAACTGAAGGTAAA, CAGGCTGGTCTGGTCAGTGTA and AAGCTTCTTTCTTGGCAATTA

HiPerfect transfection reagent (Qiagen Pty Ltd, Vic, Australia) and DMEM (with no serum or antibiotics) was added and allowed to incubate for 10 minutes at $26^{\circ} \mathrm{C}$. The siRNA complex was then added drop-wise onto cells $(115 \mu \mathrm{l} /$ well $)$ and mixed by gentle swirling, giving a final concentration of $5 \mathrm{nM}$ for each 
siRNA. The cells were incubated for 24 hours with the siRNA complex at $37^{\circ} \mathrm{C}, 5 \% \mathrm{CO}_{2}$. The medium was then changed to include $10 \%$ FBS serum supplement and the cells were further incubated for 24 hours prior to treatment. Gene knock-down was evaluated by RT-PCR. We achieved a consistent knock-down of $75-80 \%$ (data not shown). FFA3 knock-down cells and control cells were treated with and without $2 \mathrm{mM}$ sodium butyrate in high glucose DMEM containing $10 \%$ FBS and L-glutamine for 24 hours. After this time, medium and cells were removed and stored at $-80^{\circ} \mathrm{C}$ for further protein analysis.

\section{Real-time RT-PCR analysis of $m R N A$ expression}

Total RNA was extracted from frozen $\left(-80^{\circ} \mathrm{C}\right)$ undifferentiated and differentiated cell samples using RNeasy extraction kits (Qiagen Pty Ltd, Vic, Australia). Samples were treated with DNase as per instructions. mRNA was quantified using a Nanodrop spectrophotometer (Nanodrop Technologies, USA). Real-time RT-PCR was performed on a Corbett Rotor Gene 6 using SYBR Green and ROX. The reactions were performed in a $15 \mu \mathrm{l}$ volume containing $38 \mathrm{ng}$ of the extracted RNAs, 7.5 $\mu$ l of RT-PCR Master Mix, $0.15 \mu \mathrm{l}$ of RT Mix, and $0.6 \mu l$ of the appropriate primers (SDF-1, FFA3, FFA2, PPIA, MAPK, or Pref-1). Primers were from Jomar Diagnostics Pty Ltd, SA, Australia. The reactions were normalised against peptidylpropyl isomerase A (PPI). CT values were plotted for each treatment group to determine the differences in expression level between groups.

\section{ELISA for SDF-1}

SDF-1 protein secretion was analysed using Quantikine ELISA immunoassay (Bioscientific Pty Ltd, NSW, Australia) according to manufacturer's instructions. Cell culture supernatant (conditioned medium) was centrifuged at $12,000 \mathrm{~g}$ for 15 minutes at $4^{\circ} \mathrm{C}$. Addition of triplicate samples to the pre-coated SDF-1 plate was followed by 2 hours incubation at room temperature on an orbital shaker. The samples were then washed, followed by addition of SDF-1 $\alpha$ HRP-conjugate (2 hours) and a subsequent wash. Substrate solution was added and the plate incubated for 30 minutes at room temperature. Stop solution was then added and optical density measured within 30 minutes at $540 \mathrm{~nm}$ using Tecan Sunrise ${ }^{\mathrm{TM}}$ (Tecan
Trading AG, Switzerland) plate reader at $450 \mathrm{~nm}$ without correction.

\section{Statistical Analysis}

RT-PCR and ELISA data were analysed using Graphpad Prism ${ }^{\mathrm{TM}}$ software. Data were checked for normality using the Anderson-Darling Normality test. Based on the outcome of the test, parametric or non-parametric tests were carried out. Statistical comparisons between treatments were calculated using one-way analysis of variance (ANOVA) followed by Tukey's post hoc test corrected for multiple comparisons. Unpaired student t-test was used for direct comparison of two sets of data. Results are expressed as mean \pm standard error of the mean (SEM) normalised as percentages of control unless otherwise stated. $\mathrm{p}<0.05$ was deemed to be statistically significant.

\section{RESULTS}

\section{Butyrate increases SDF-1 expression and protein secretion by 3T3-L1 cells}

As expected, exposure of 3T3-L1 pre-adipocytes to IBMX medium led to cell differentiation, as evidenced by decreased expression of the pre-adipocyte marker Pref-1 (Figure 1A, $p=0.002$ ) and considerable accumulation of lipid droplets (Oil red O staining, Figure 1B). SDF-1 mRNA was abundantly expressed in pre-adipocytes and its expression was significantly increased in the presence of butyrate in a time-dependent manner (Figure 1C, $\mathrm{p}<0.001$ ). In replicate experiments, SDF-1 mRNA was increased up to 6-fold over 24 hours. Maximal stimulation was obtained with $2 \mathrm{mM}$ butyrate. There was no detectable stimulation at $0.2 \mathrm{mM}$ nor once concentrations reached $10 \mathrm{mM}$ (data not shown). Butyrate also increased SDF-1 protein secretion from 3T3-L1 cells by 2.4-fold over 24 hours (Figure 1D, p <0.001). SDF-1 secretion was also apparent from differentiated 3T3-L1 cells, but there was no detected increase in secretion following exposure to butyrate.

\section{The effect of butyrate is not mediated through HDAC inhibition}

Both sodium valproate $(2 \mathrm{mM})$ and the specific HDACi, trichostatin, modestly decreased SDF-1 secretion from differentiated 3T3-L1 cells (Figure 
$1 \mathrm{D}, \mathrm{p}=0.001$ and $\mathrm{p}=0.047$, respectively). Compared with butyrate, there was enhanced SDF-1 secretion from 3T3-L1 pre-adipocytes in the presence of acetate $(2 \mathrm{mM}, \mathrm{p}=0.002)$. Propionate $(2 \mathrm{mM}, \mathrm{p}<0.001)$ was even more potent than butyrate (Figure 1E). The

A

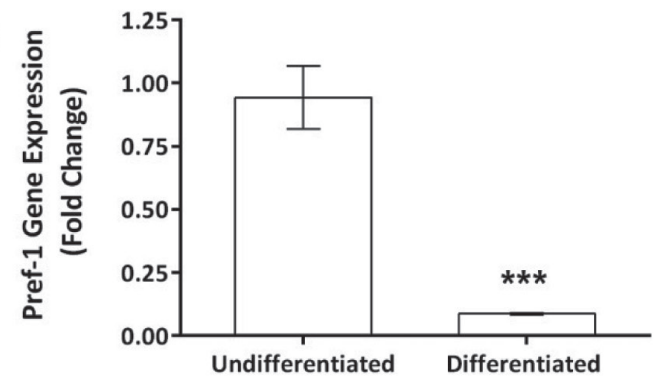

C

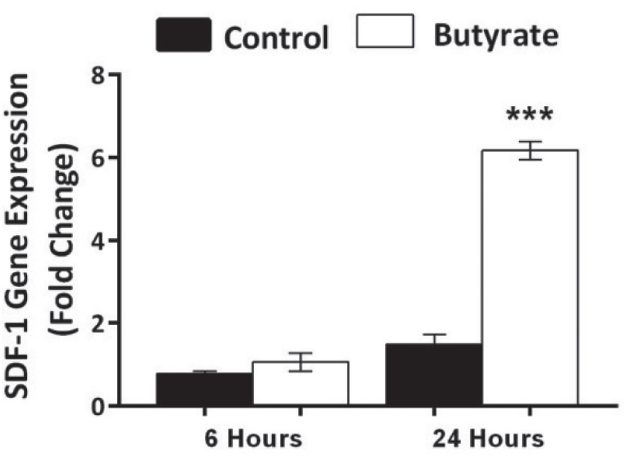

E

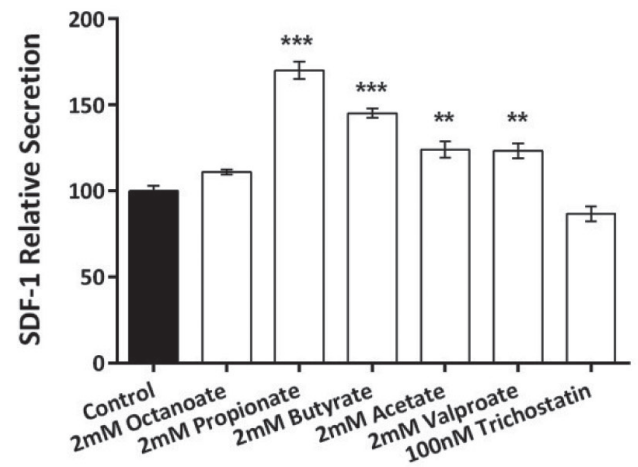

order of potency of the three SCFAs was propionate (C3) $>$ butyrate $(\mathrm{C} 4)>$ acetate $(\mathrm{C} 2)$, consistent with an action through FFA3. Trichostatin decreased the expression of SDF- 1 at concentrations as low as $1 \mu \mathrm{M}$ (Figure 1F, p=0.003).
B

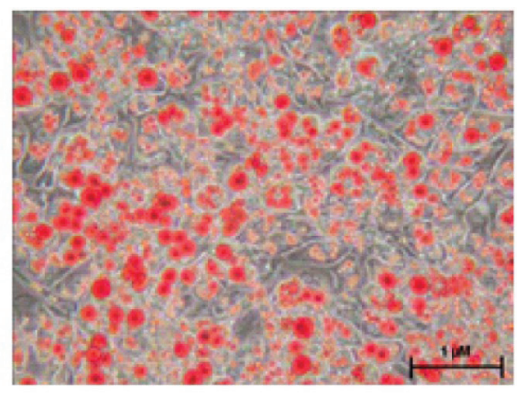

D

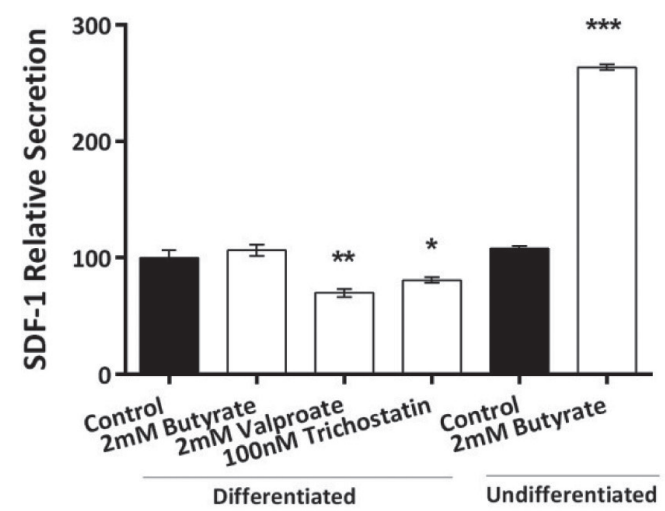

F

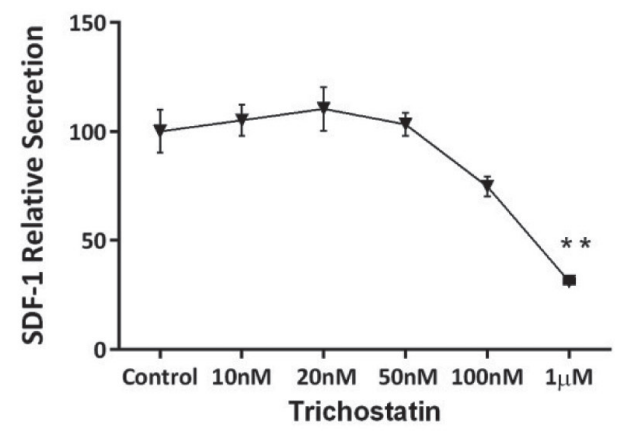

Figure 1. Butyrate increases SDF-1 expression and protein secretion by 3T3-L1 cells. A: Pref-1 expression in 3T3-L1 adipocytes. 3T3-L1 pre-adipocytes expressed high levels of Pref-1. Expression was markedly decreased when the cells were differentiated (t-test, $\mathrm{p}<0.001)$. B: Differentiated 3T3-L1 adipocytes, showing numerous lipid droplets stained with Oil red O. Photograph was taken 10 days after exposure of 3T3 cells to IDX medium. C: Butyrate induced a marked increase in SDF-1 mRNA determined when cells were incubated with $2 \mathrm{mM}$ butyrate (ANOVA, $\mathrm{p}<0.001$; control vs Butyrate $24 \mathrm{Hrs}, \mathrm{p}<0.001$ ). D: Effect of HDACi (ANOVA: $\mathrm{p}$ $<0.0001$ ): In adipocytes, trichostatin and valproate decreased secretion of SDF-1, while butyrate was without effect. This contrasts with the stimulatory effect of butyrate in pre-adipocytes. E: Comparison of SCFA and HDACi on pre-adipocytes (ANOVA, $p$ $<0.001$ ): Order of potency of SCFA was C3 > C4 > C2. HDACi were without effect. F: Dose-response curve for the inhibitory effect of trichostatin on SDF-1 secretion from adipocytes (ANOVA, $p<0.001$ ). $*$ : $<<0.05 ; * *: p<0.01 ; * * *: p<0.001$. Data shown are the mean \pm SEM normalised as percentage of control. Differentiated cells = mature adipocytes; Undifferentiated cells= pre-adipocytes. 


\section{The effect of butyrate is mediated through the FFA3 receptor}

Pertussis toxin (PTX), which blocks GPR signalling, was used to investigate the potential role of GPR in mediating the above stimulatory effect of butyrate. When used alone at concentrations of $5 \mathrm{nM}$, PTX had no effect on SDF-1 secretion by 3T3-L1 pre-adipocytes (Figure 2A, $\mathrm{p}=0.009$ ). When added along with butyrate, PTX markedly decreased the
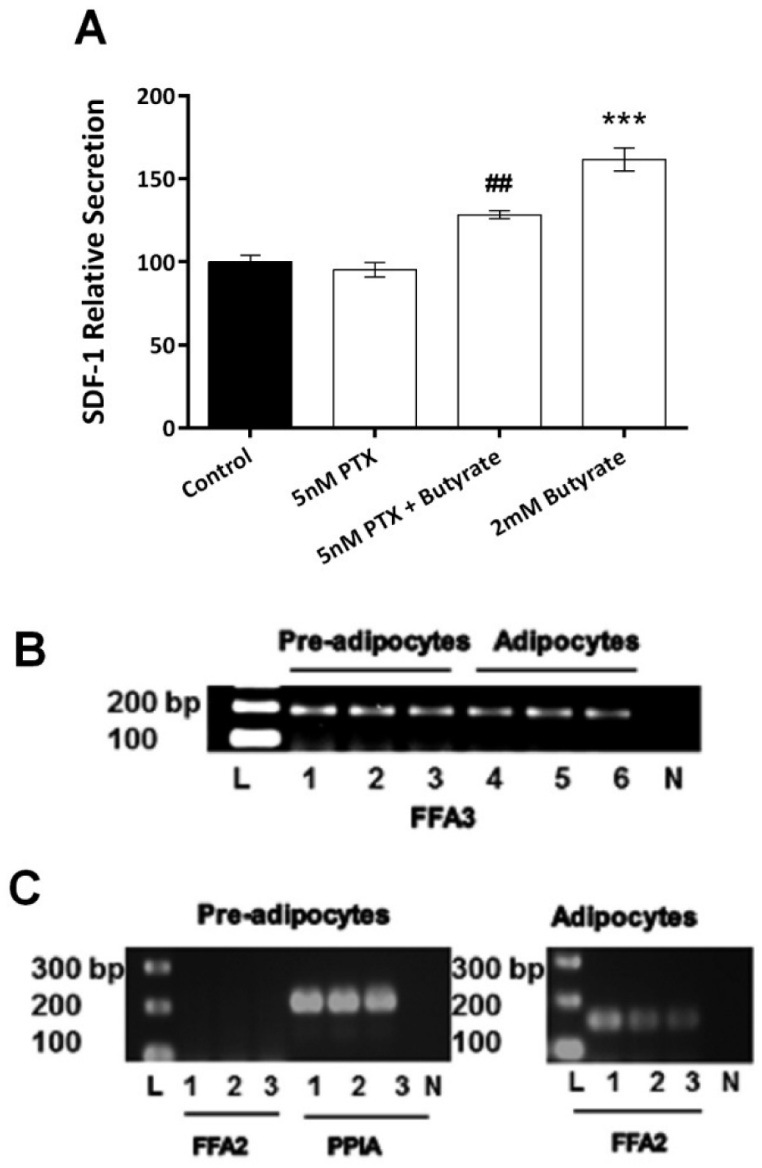

Figure 2. Pertussis toxin (PTX) abolishes the stimulatory effects of butyrate, and FFA2/FFA3 expression. A: PTX alone was without effect on SDF-1 secretion from pre-adipocytes (ANOVA, $\mathrm{p}<0.001$ ). The stimulatory effect of butyrate was decreased in the presence of $5 \mathrm{nM}(\# \#=\mathrm{p}<0.01$, compared against 2M Butyrate) PTX. Data shown are the mean \pm SEM normalised as percentage of control. $\mathrm{B}+\mathrm{C}$ : Shows $2 \%$ agarose gel electrophoresis and ethidium bromide staining. B: Expression of FFA3 (188 bp). L = ladder. Lanes 1-3 = 3T3-L1 are pre-adipocytes. Lanes 4-6 are 3T3-L1 adipocytes. $\mathrm{N}=$ no reverse-transcriptase negative control. C: Expression of FFA2 (156bp) - no expression was found in pre-adipocytes (lanes 1-3), while the housekeeping gene PPIA (200bp) was strongly expressed. FFA2 was expressed in differentiated adipocytes. stimulatory effect of the former on SDF-1 secretion. As there are two receptors for SCFA (FFA2 and FFA3), we investigated the relative expression of these receptors in pre-adipocytes and adipocytes using RT-PCR. FFA3 was expressed in both pre-adipocytes and in adipocytes (Figure 2B), while FFA2 was only expressed in differentiated adipocytes (Figure 2C).

Using siRNA for FFA3, we successfully decreased gene expression to less than $30 \%$ of its baseline value in 3T3-L1 pre-adipocytes (Figure 3A, $\mathrm{p}=0.012$ ) While the stimulatory effect of butyrate on SDF-1 mRNA was apparent in control cells, this effect was entirely lost in cells treated with siRNA for FFA3 (Figure 3B). Similarly, although butyrate increased SDF-1 secretion from pre-adipocytes, there was no such stimulation in cells in which the FFA3 gene had been silenced (Figure 3C).

\section{SDF-1 is a potential mediator of the interaction between pre-adipocytes and macrophages}

We determined the level of SDF-1 expression and its obligate receptor (CXCR4) on cells of the mouse monocyte/macrophage line J774.2 compared with 3T3-L1 pre-adipocytes when treated with SCFA propionate (C3). Propionate was chosen as it elicited the highest SDF-1 secretion amongst the SCFAs (Figure 1E). SDF-1 protein secretion was higher in pre-adipocytes when treated with propionate compared with controls (Figure 4A, $p=0.017$ ). In contrast, SDF-1 secretion from J774.2 cells was very low and there was no significant stimulation with propionate (Figure 4A). 3T3-L1 pre-adipocytes expressed abundant SDF-1 mRNA, while there were much lower levels of expression in $\mathbf{J} 774.2$ cells (Figure 4B). On the other hand, CXCR4 expression was not detected in pre-adipocytes, but mRNA for CXCR4 was abundant in J774.2 cells (Figure 4C).

\section{Human pre-adipocytes also express SDF-1 and the FFA3 receptor}

It is beyond the scope of this study to replicate all of the above work with human cells. However, SDF-1 mRNA expression was noted in human pre-adipocytes, and there was a significant increase in expression when cells were incubated in the presence of $2 \mathrm{mM}$ butyrate (Figure 5A, $\mathrm{p}=0.034$ ). Using immunocytochemistry, we detected expression of FFA3, but not FFA2, in cultured human pre-adipocytes (Figure 5B-C). Both 

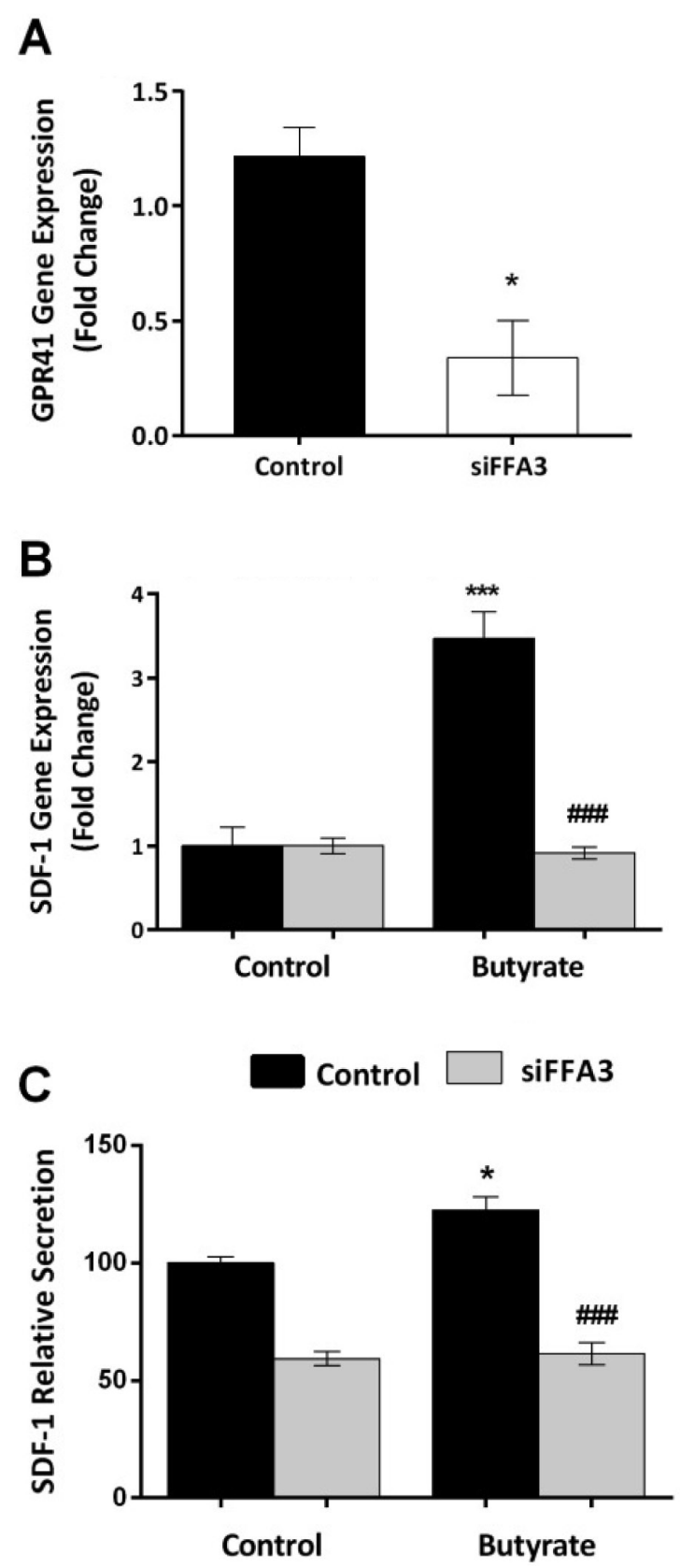

Figure 3. siRNA for FFA3 abolishes the stimulatory effect of butyrate. A: siFFA3 gene knockdown, evaluated by RT-PCR reached $75-80 \%$ (t-test, $\mathrm{p}<0.05$ ). Data shown are the mean \pm SEM. B: SDF-1 m-RNA expression increased when cells were treated with butyrate (ANOVA, $\mathrm{p}<0.001$, ***: $\mathrm{p}<0.001$ compared with control). This stimulatory effect was markedly decreased when the FFA3 gene was silenced (\#\#\#: p <0.001 compared with butyrate treatment of control cells). C: SDF-1 protein secretion was increased when control cells were treated with butyrate (ANOVA, $\mathrm{p}<0.001,{ }^{*}$ : $\mathrm{p}<0.05$ ). This stimulation was markedly decreased following FFA3 gene silencing (\#\#\#: $\mathrm{p}<0.001)$. Data shown are the mean \pm SEM normalised as percentage of control (for B \& C).
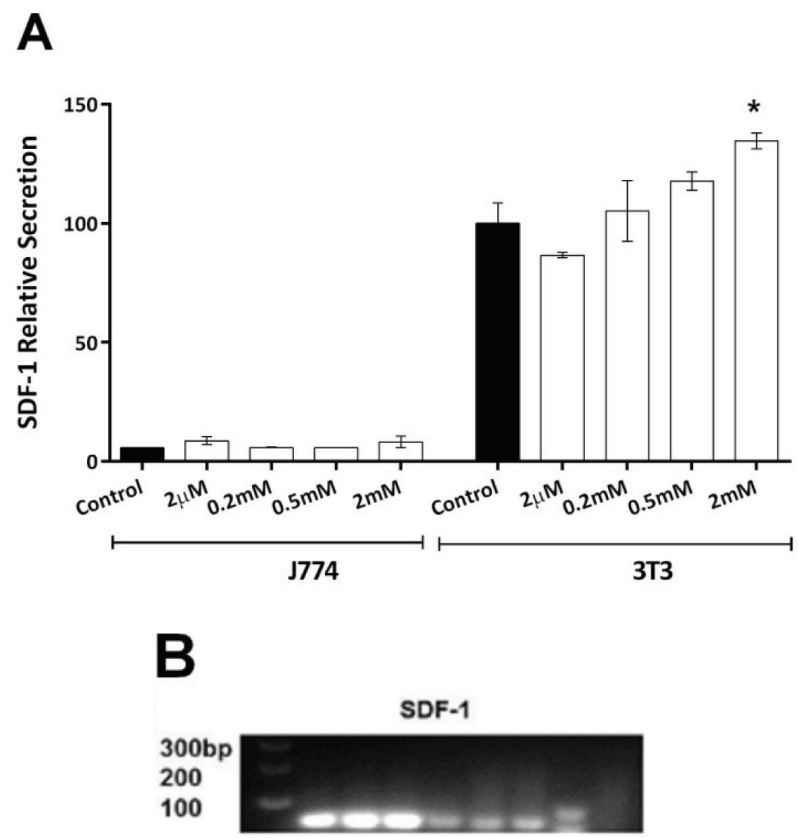

L C1 C2 C3 T1 T2 T3 N1 N2

Pre-adipocytes $\overline{\mathrm{J} 774.2}$

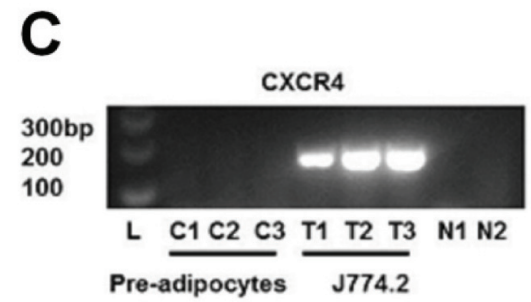

Figure 4. Effect of Propionate on SDF-1 secretion. A: Propionate enhanced SDF-1 protein secretion from pre-adipocytes, but there was only minimal SDF-1 secretion and no effect of propionate in cultures of J774.2 cells (ANOVA, p <0.001). Data shown are the mean \pm SEM normalised as percentage of control. *: p <0.05. B + C: $2 \%$ Agarose gel electrophoresis with ethidium bromide staining. L: ladder; N1: no template negative control; N2: no reverse-transcriptase negative control. SDF-1 (57bp) was detected in pre-adipocytes but not highly expressed in J774.2 cells. CXCR4 (156bp) expression was not detected in pre-adipocytes, but mRNA for CXCR4 was abundant in J774.2 cells.

FFA2 and FFA3 were expressed on cells of the human monocyte/macrophage line THP-1 (Figure 5D-E).

\section{DISCUSSION}

In this study, we have shown that SDF-1 (CXCL12) is expressed in, and secreted by, 3T3-L1 cells. This expression is particularly evident in the pre-adipocyte 


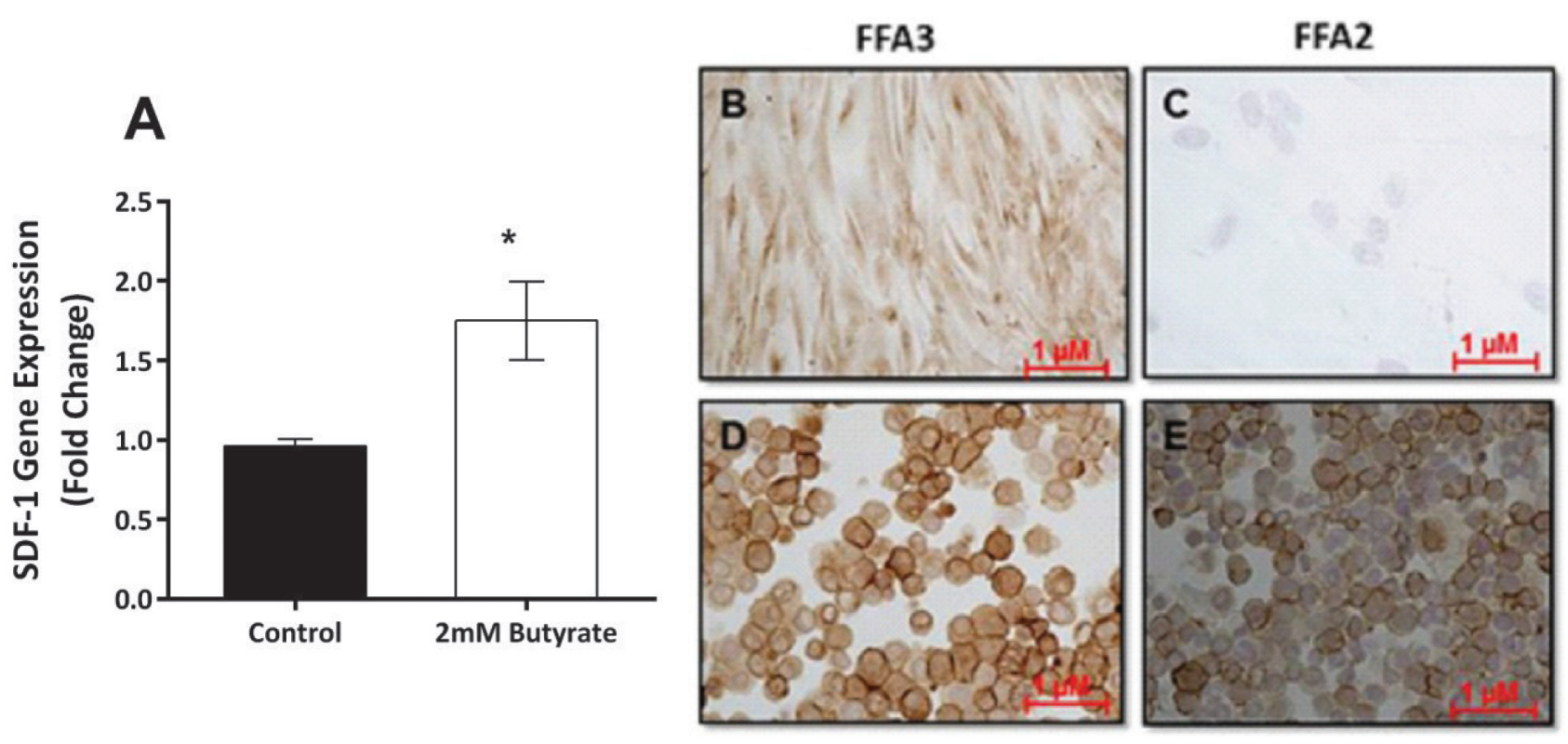

Figure 5. Human pre-adipocytes express SDF-1 and FFA3. A: SDF-1 mRNA expression was abundant in human pre-adipocytes, and this expression increased when cells were incubated with $2 \mathrm{mM}$ butyrate (t-test, $\left.{ }^{*}: \mathrm{p}<0.05\right)$. B: Expression of FFA3 in human preadipocytes. C: No expression of FFA2 in human pre-adipocytes. D: Expression of FFA3 in cells of the human monocyte/macrophage line THP-1. E: Expression of FFA2 in THP-1 cells.

state. We have also shown that SDF-1 mRNA and protein are increased by treatment with SCFA. The effect of SCFA was not replicated with the HDACs valproate and trichostatin and the inhibitory effect of pertussis toxin suggests that SCFA effects are mediated through a $\mathrm{G}$ protein-coupled receptor. The order of potency of SCFA $(\mathrm{C} 3>\mathrm{C} 4>\mathrm{C} 2)$ suggests an effect through FFA3 rather than FFA2. Indeed FFA3, but not FFA2, was expressed in pre-adipocytes. Silencing of the FFA3 gene abolished the stimulatory effect of SCFA.

Recent studies show that SDF-1 plays a central role in the pathogenesis of diabetic retinopathy. ${ }^{27,28}$ SDF-1, through interaction with the CXCR4 receptor on bone marrow-derived vascular progenitor cells, is also crucial in vascular modelling after ischaemia. ${ }^{17,29,30}$ Decreased SDF-1 secretion in patients with diabetes is also understood to contribute to defective wound healing, including in diabetic foot ulcers. ${ }^{31}$ It is likely that cells of the stromal vascular compartment of adipose tissue are a major source of SDF-1., ${ }^{9,32}$ In obesity, these cells may be depleted due to differentiation of pre-adipocytes into mature fat-laden cells. The predominance of differentiated and hypertrophied adipocytes leads to secretion of me- diators that contribute to systemic inflammation and insulin resistance. ${ }^{33}$ Our finding that pre-adipocytes are a source of SDF-1 confirms earlier observations that the chemokine is expressed in these cells. ${ }^{20-22}$

The HDACs are a group of around 18 enzymes involved in epigenetic regulation of gene expression. HDAC inhibitors are considered potential treatments for neoplasia and neurodegenerative disorders, and as angiogenesis inhibitors. Butyrate is a naturally occurring HDAC inhibitor. While HDACs are involved in adipogenesis, ${ }^{34,35}$ the effects of HDAC inhibitors in this process remain uncertain. Valproate has been reported to inhibit adipogenesis, ${ }^{36}$ while diallyl disulphide, contained in garlic, may stimulate adipogenesis. ${ }^{37} \mathrm{SCF} A$ have been reported to promote adipocyte differentiation and expression of adipogenic markers. ${ }^{5,38,39}$ In this study, we found that the potent HDAC inhibitor, trichostatin, inhibited SDF-1 expression in pre-adipocytes. Conversely, butyrate increased SDF-1 expression and secretion. The stimulatory effect of butyrate on SDF-1 expression and secretion was observed with other SCFAs that have only weak HDAC inhibitory activity.

In mice, plasma leptin concentration was increased by oral administration of propionate..$^{8}$ The circulating 
propionate concentration achieved was comparable to what we used in vitro. Most of the butyrate generated within the colon is removed in the enterohepatic circulation, while high levels of acetate and propionate can reach the systemic circulation. Propionate levels are particularly high after consumption of certain functional foods, including barley kernels, ${ }^{40}$ and acetate levels also increase after alcohol consumption. ${ }^{41}$ We do not, therefore, feel that the concentrations of SCFA used in our experiments were unduly high. Additionally, we do not know what the most potent naturally occurring ligands for FFA3 are.

Given their role as part of the body's nutrient sensing apparatus, it is not surprising to find receptors for SCFA expressed in cells of adipocyte lineage. Our results disagree with those of Hong et $\mathrm{al}^{3}$ who did not find FFA 3 expression in mouse adipose depots. They did report increased adipogenesis with acetate and propionate acting through FFA2. We, in contrast, found FFA3 to be expressed in both pre-adipocytes and adipocytes, while FFA2 was only expressed in adipocytes. Xiong et $\mathrm{al}^{8}$ described the role of FFA3 in adipocyte leptin secretion induced by SCFAs. We demonstrate here that the effect of SCFA on SDF-1 was abolished with pertussis toxin, suggesting that it is GPR-mediated. We then examined the role of FFA3 in regulating SDF-1 expression. FFA3 gene silencing abolished the stimulatory effect of butyrate on SDF-1 expression and protein secretion. We also confirmed that SCFA increase SDF-1 expression in human pre-adipocytes and that FFA3, but not FFA2, is expressed in these cells.

The biological actions of SDF-1 are through its obligate receptor CXCR4 (also a co-receptor for lymphotrophic strains of the HIV virus). We did not find CXCR4 to be expressed in either pre-adipocytes or adipocytes, making it unlikely that SDF-1 and CXCR 4 interaction is involved in cross-talk between mature adipocytes and stromal cells. Others have reported CXCR4 expression in adipocytes, allowing them to be infected with $\mathrm{HIV},{ }^{42}$ a finding not confirmed by a subsequent study..$^{43}$ As expected, we detected abundant expression of CXCR4 in mouse $\mathbf{J} 774.2$ macrophages, but there was very little SDF-1 expression in these cells, and no effect of added propionate on SDF-1 expression. It seems highly likely therefore that SDF-1 and CXCR 4 are involved in the interaction between adipocytes and macrophages, although somewhat surprisingly this has not been studied to date.

We propose that the SDF-1 secreted by adipocytes and its interaction with CXCR4 receptor on tissue macrophages mediates interaction between the two cell types (Figure 6). SDF-1 secretion by pre-adipocytes may increase monocyte infiltration into adipose and factors secreted by monocytes may inhibit adipocyte differentiation. ${ }^{10,44}$ In states of fat excess, such impaired differentiation may lead to ectopic deposition of fat, including in the vascular wall. On the other hand, hypertrophied ${ }^{33}$ or $\operatorname{dead}^{45}$ adipocytes attract macrophages and such infiltration in visceral fat partly accounts for the association between visceral obesity and systemic inflammation and insulin resistance. ${ }^{46,47}$

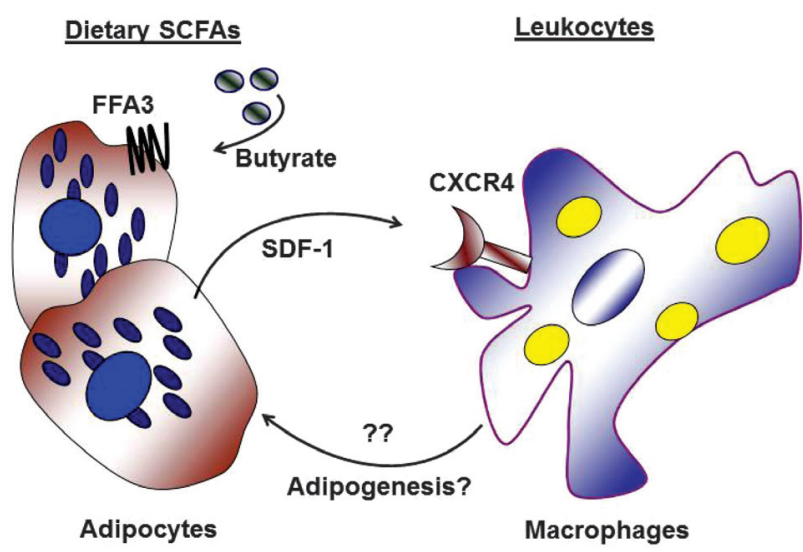

Figure 6. Hypothetical adipocyte-macrophage interactions. Dietary SCFAs including butyrate induce adipocytes to secrete SDF-1, acting through the FFA3 receptor. Secreted SDF-1 attracts other cells including macrophages, interacting through its receptor CXCR-4. This adipocyte-macrophage interaction could initiate secretion of mediators from macrophages, possibly regulating processes associated with adipogenesis and insulin resistance.

\section{CONCLUSIONS}

In conclusion, we have demonstrated that SDF1 (CXCL12) is secreted by 3T3-L1 pre-adipocytes and adipocytes and that this is regulated by SCFA in a mechanism that does not depend on histone deacetylase inhibition. Our data strongly suggest that the effect of SCFA is mediated through FFA3. 
SDF-1 may be an important mediator of the interaction between adipocytes and bone marrow-derived cells including monocyte/macrophage cells. These observations shed light on a novel pathway mediating interaction between adipocyte precursors and inflammatory cells. Further studies may increase our understanding of the processes involved in adipose depot remodelling and the systemic inflammation that accompanies obesity.

\section{ACKNOWLEDGEMENTS}

We are grateful to Frances Wood, Monsur Kazi, and Stephen Garland for their technical expertise and contribution to this work.

We would like also to thank Dr. Mark Vucak, Consultant Plastic Surgeon, Mater Hospital, Townsville, who kindly supplied the samples from which human pre-adipocytes were cultured.

\section{FUNDING}

We appreciate the support of James Cook University and the Private Practice Fund of the Townsville Hospital, Queensland, Australia for funding this research.

\section{REFERENCES}

1. Brown AJ, Jupe S, Briscoe CP, 2005 A family of fatty acid binding receptors. DNA Cell Biol 24: 54-61.

2. Covington DK, Briscoe CA, Brown AJ, Jayawickreme CK, 2006 The G-protein-coupled receptor 40 family (GPR40-GPR43) and its role in nutrient sensing. Biochem Soc Trans 34: 770-773.

3. Hong YH, Nishimura Y, Hishikawa D, et al, 2005 Acetate and propionate short chain fatty acids stimulate adipogenesis via GPCR43. Endocrinology 146: 50925099.

4. Ge H, Li X, Weiszmann J, et al, 2008 Activation of $\mathrm{G}$ protein-coupled receptor 43 in adipocytes leads to inhibition of lipolysis and suppression of plasma free fatty acids. Endocrinology 149: 4519-4526.

5. Zaibi MS, Stocker CJ, O'Dowd J, et al, 2010 Roles of GPR41 and GPR43 in leptin secretory responses of murine adipocytes to short chain fatty acids. FEBS Lett 584: 2381-2386.

6. Tolhurst G, Heffron H, Lam YS, et al, 2012 Short-chain fatty acids stimulate glucagon-like peptide-1 secretion via the G-protein-coupled receptor FFAR2. Diabetes 61: 364-371.
7. Dewulf EM, Ge Q, Bindels LB, et al, 2013 Evaluation of the relationship between GPR43 and adiposity in human. Nutr Metab (Lond) 10: 11.

8. Xiong Y, Miyamoto N, Shibata K, et al, 2004 Short-chain fatty acids stimulate leptin production in adipocytes through the G protein-coupled receptor GPR41. Proc Natl Acad Sci U S A 101: 1045-1050.

9. Cho CH, Koh YJ, Han J, et al, 2007 Angiogenic role of LYVE-1-positive macrophages in adipose tissue. Circ Res 100: e47-57.

10. Lacasa D, Taleb S, Keophiphath M, Miranville A, Clement K, 2007 Macrophage-secreted factors impair human adipogenesis: involvement of proinflammatory state in preadipocytes. Endocrinology 148: 868-877.

11. Oh DY, Lagakos WS, 2011 The role of G-proteincoupled receptors in mediating the effect of fatty acids on inflammation and insulin sensitivity. Curr Opin Clin Nutr Metab Care 14: 322-327.

12. Permana PA, Menge C, Reaven PD, 2006 Macrophagesecreted factors induce adipocyte inflammation and insulin resistance. Biochem Biophys Res Commun 341: 507-514.

13. Harford KA, Reynolds CM, McGillicuddy FC, Roche HM, 2011 Fats, inflammation and insulin resistance: insights to the role of macrophage and T-cell accumulation in adipose tissue. Proc Nutr Soc 70: 408-417.

14. Maslowski KM, Vieira AT, Ng A, et al, 2009 Regulation of inflammatory responses by gut microbiota and chemoattractant receptor GPR43. Nature 461: 1282-1286.

15. Tian J, Dang HN, Yong J, et al, 2011 Oral treatment with y-aminobutyric acid improves glucose tolerance and insulin sensitivity by inhibiting inflammation in high fat diet-fed mice. PLoS ONE 6: e25338.

16. Marsland BJ, 2012 Regulation of inflammatory responses by the commensal microbiota. Thorax 67: 93-94.

17. Ghadge SK, Muhlstedt S, Ozcelik C, Bader M, 2011 SDF-1 as a therapeutic stem cell homing factor in myocardial infarction. Pharmacol Ther 129: 97-108.

18. Liu G, Lu P, Li L, et al, 2011 Critical role of SDF1-induced progenitor cell recruitment and macrophage VEGF production in the experimental corneal neovascularization. Mol Vis 17: 2129-2138.

19. Ji RC, 2012 Macrophages are important mediators of either tumor- or inflammation-induced lymphangiogenesis. Cell Mol Life Sci 69: 897-914.

20. Burton GR, Guan Y, Nagarajan R, McGehee RE Jr, 2002 Microarray analysis of gene expression during early adipocyte differentiation. Gene 293: 21-31.

21. Gerhold DL, Liu F, Jiang G, et al, 2002 Gene expression profile of adipocyte differentiation and its regulation by peroxisome proliferator-activated receptor-gamma agonists. Endocrinology 143: 2106-2118.

22. Kratchmarova I, Kalume DE, Blagoev B, et al, 2002 A proteomic approach for identification of secreted proteins during the differentiation of 3T3-L1 preadipocytes to adipocytes. Mol Cell Proteomics 1: 213-222. 
23. Oh I, Ozaki M, Miyazato A, et al, 2007 Screening of genes responsible for differentiation of mouse mesenchymal stromal cells by DNA micro-array analysis of C3H10T1/2 and C3H10T1/2-derived cell lines. Cytotherapy 9: 80-90.

24. Blogowski W, Budkowska M, Salata D, et al, 2013 Clinical analysis of selected complement-derived molecules in human adipose tissue. J Transl Med 11: 11.

25. Jordan NJ, Kolios G, Abbot SE, et al, 1999 Expression of functional CXCR4 chemokine receptors on human colonic epithelial cells. J Clinl Invest 104: 1061-1069.

26. Shibuta K, Mori M, Shimoda K, et al, 2002 Regional expression of CXCL12/CXCR4 in liver and hepatocellular carcinoma and cell-cycle variation during in vitro differentiation. Jpn J Cancer Res 93: 789-797.

27. Butler JM, Guthrie SM, Koc M, et al, 2005 SDF-1 is both necessary and sufficient to promote proliferative retinopathy. J Clin Invest 115: 86-93.

28. Abu El-Asrar AM, Struyf S, Kangave D, Geboes K, Van Damme J, 2006 Chemokines in proliferative diabetic retinopathy and proliferative vitreoretinopathy. Eur Cytokine Netw 17: 155-165.

29. Gallagher KA, Liu ZJ, Xiao M, et al, 2007 Diabetic impairments in NO-mediated endothelial progenitor cell mobilization and homing are reversed by hyperoxia and SDF-1 alpha. J Clin Invest 117: 1249-1259.

30. van Weel V, Seghers L, de Vries MR, et al, 2007 Expression of vascular endothelial growth factor, stromal cell-derived factor-1, and CXCR4 in human limb muscle with acute and chronic ischemia. Arterioscler Thromb Vasc Biol 27: 1426-1432.

31. Di Rocco G, Gentile A, Antonini A, et al, 2010 Enhanced healing of diabetic wounds by topical administration of adipose tissue-derived stromal cells overexpressing stromal-derived factor-1: biodistribution and engraftment analysis by bioluminescent imaging. Stem Cells Int 2011: 304562.

32. Thangarajah H, Vial IN, Chang E, et al, 2009 IFATS collection: Adipose stromal cells adopt a proangiogenic phenotype under the influence of hypoxia. Stem Cells 27: 266-274.

33. Skurk T, Alberti-Huber C, Herder C, Hauner H, 2007 Relationship between adipocyte size and adipokine expression and secretion. J Clin Endocrinol Metab 92: 1023-1033.

34. Fajas L, Egler V, Reiter R, et al, 2002 The retinoblastoma-histone deacetylase 3 complex inhibits PPARgamma and adipocyte differentiation. Dev Cell 3: 903-910.

35. Li D, Yea S, Li S, et al, 2005 Kruppel-like factor-6 promotes preadipocyte differentiation through histone deacetylase 3-dependent repression of DLK1. J Biol Chem 280: 26941-26952.

36. Lagace DC, Nachtigal MW, 2004 Inhibition of histone deacetylase activity by valproic acid blocks adipogenesis. J Biol Chem 279: 18851-18860.

37. Lee JH, Kim KA, Kwon KB, et al, 2007 Diallyl disulfide accelerates adipogenesis in 3T3-L1 cells. Int J Mol Med 20: $59-64$.

38. Toscani A, Soprano DR, Soprano KJ, 1990 Sodium butyrate in combination with insulin or dexamethasone can terminally differentiate actively proliferating Swiss 3T3 cells into adipocytes. J Biol Chem 265: 5722-5730.

39. Yoo EJ, Chung JJ, Choe SS, Kim KH, Kim JB, 2006 Down-regulation of histone deacetylases stimulates adipocyte differentiation. J Biol Chem 281: 6608-6615.

40. Nilsson A, Granfeldt Y, Ostman E, Preston T, Bjorck I, 2006 Effects of GI and content of indigestible carbohydrates of cereal-based evening meals on glucose tolerance at a subsequent standardised breakfast. Eur J Clin Nutr 60: 1092-1099.

41. Sarkola T, Iles MR, Kohlenberg-Mueller K, Eriksson CJ, 2002 Ethanol, acetaldehyde, acetate, and lactate levels after alcohol intake in white men and women: effect of 4-methylpyrazole. Alcohol Clin Exp Res 26: 239-245.

42. Hazan U, Romero IA, Cancello R, et al, 2002 Human adipose cells express CD4, CXCR4, and CCR5 [corrected] receptors: a new target cell type for the immunodeficiency virus-1? Faseb J 16: 1254-1256.

43. Munier S, Borjabad A, Lemaire M, Mariot V, Hazan U, 2003 In vitro infection of human primary adipose cells with HIV-1: a reassessment. Aids 17: 2537-2539.

44. Constant VA, Gagnon A, Landry A, Sorisky A, 2006 Macrophage-conditioned medium inhibits the differentiation of 3T3-L1 and human abdominal preadipocytes. Diabetologia 49: 1402-1411.

45. Cinti S, Mitchell G, Barbatelli G, et al, 2005 Adipocyte death defines macrophage localization and function in adipose tissue of obese mice and humans. J Lipid Res 46: 2347-2355.

46. Cancello R, Tordjman J, Poitou C, et al, 2006 Increased infiltration of macrophages in omental adipose tissue is associated with marked hepatic lesions in morbid human obesity. Diabetes 55: 1554-1561.

47. Okamoto Y, Higashiyama H, Rong JX, et al, 2007 Comparison of mitochondrial and macrophage content between subcutaneous and visceral fat in $\mathrm{db} / \mathrm{db}$ mice. Exp Mol Pathol 83: 73-83. 


\title{
A systematic review and meta-analysis of weight status among adolescents in Cyprus: scrutinizing the data for the years 2000-2010
}

\author{
Maria G. Grammatikopoulou, ${ }^{1}$ Eleni P. Kotanidou, ${ }^{2}$ Anastasia G. Markaki, ${ }^{3}$ \\ Charilaos Stylianou, ${ }^{4}$ Assimina Galli-Tsinopoulou, ${ }^{2}$ Maria Tsigga, ${ }^{1}$ Maria Hassapidou ${ }^{1}$
}

${ }^{1}$ Department of Nutrition \& Dietetics, Alexander Technological Educational Institute, Thessaloniki; ${ }^{2} 4^{\text {th }}$ Department of Pediatrics, Faculty of Medicine, Aristotle University of Thessaloniki, Papageorgiou General Hospital, Thessaloniki; ${ }^{3}$ Department of Nutrition \& Dietetics, Technological Educational Institute of Crete, Crete; Greece; ${ }^{4}$ Department of Pediatrics, Paphos General Hospital, Anavargos, Paphos, Cyprus

\begin{abstract}
OBJECTIVE: The aim of the present study was to evaluate by pooled and sensitivity analyses all available data on adolescent overweight/obesity in Cyprus. DESIGN: A thorough literature search determined the studies to be examined using Cypriot adolescent samples aged 10-18 years old, with weight status in each sex classified according to the IOTF criteria, published between the years 2001-2011. Eight studies were retrieved, but three fulfilled the criteria for the sensitivity analyses. RESULTS: The pooled prevalence of obesity was $9.8 \%$ in boys $(n=6081)$. The pooled analysis classified $6.1 \%(n=3886)$ of girls as obese, whereas a higher prevalence was observed by the sensitivity analysis $6.4 \%(n=1956, p \leq 0.001)$. The boys' prevalence of overweight was $19.3 \%$ and the girls' $17.1 \%$. Between sexes, boys demonstrated a higher prevalence of obesity and overweight ( $\mathbf{p} \leq \mathbf{0 . 0 0 1}$ for both). The cumulative analyses demonstrated an increase in the prevalence of overweight/obesity until the year 2005 and thereafter a plateauing in boys and a slight decrease in girls in a non-linear manner. CONCLUSION: Approximately 1/3 of adolescent boys and $1 / 4$ of adolescent girls in Cyprus were overweight/obese during the previous decade.
\end{abstract}

Key words: Abdominal obesity, Adolescence, BMI, IOTF, Obese, Obesity, Overweight, Teenagers

\section{INTRODUCTION}

The increased prevalence of childhood obesity in Europe during the last few decades has evolved into an

Address for correspondence:

Maria Hassapidou, Department of Nutrition \& Dietetics, Alexander Technological Educational Institute, Thessaloniki, Sindos P.O. Box 141, GR-57400, Greece;

Tel./Fax: +30 2310 791584; E-mail: mnhass@gmail.com

Received 14-01-2014, Accepted 08-05-2014 important public health issue with epigenetic effects on the health of future adult generations. ${ }^{1}$ However, adolescent obesity is equally important as it represents an age-range closer to adulthood, thus depicting the imminent early adulthood prevalence. In terms of psychology, overweight during adolescence is associated with unhealthy weight-control and disordered eating, actions subvened by the health-risk behavior 
encountered during adolescence. ${ }^{2,3}$ In terms of physiological growth and hormonal response, research has shown that adolescent obesity may disrupt aspects of pubertal development. ${ }^{4}$

Though geographically different from Europe, in Cyprus trends in adolescent obesity appear similar to the rest of the continent. ${ }^{5}$ Several studies have addressed the problem of obesity in Cypriot adolescents and it is acknowledged that a multifaceted public health policy approach is needed in order to combat the disease. ${ }^{6}$ However, the majority of published research includes convenience samples with wide ageranges, often without differentiating between children and adolescents, while the file-drawer problem ${ }^{7}$ (i.e. the non-publication of studies with different results from those expected) impedes understanding of the actual progression of the disease.

The objective of this review of existing data was to synthesize all published information on the weight status of Cypriot adolescents in order to help understand the progression of adolescent obesity and provide data for the development of policies for combating the disease during adolescence.

\section{EXPERIMENTAL METHODS}

\section{Literature search}

We searched Ovid MEDLINE, EMBASE, Google Scholar, Scopus and openarchives.gr for studies published prior to December 2010, using the meSH terms "adolescent OR children", "adolescence OR childhood”, "AND obesity OR overweight”, "AND Cyprus". Where applicable, the search keywords were also translated into the Greek language. In addition, the references cited in each paper were also searched for relevant studies.

\section{Inclusion and exclusion criteria}

Criteria for inclusion were 1) research on a Cypriot population, 2) age of participants between 10 and 19 years old as suggested by the World Health Organization (WHO), ${ }^{8} 3$ ) classification of underweight (UW), overweight (OW) and obesity (OB) separately, according to the International Obesity Task Force (IOTF) criteria, ${ }^{9,10} 4$ ) weight-status classification according to sex, 5) publication of the results after 2001, when the IOTF criteria were announced, and prior to December 2010 and 6) collection of the sample between 2000 and 2010.

Studies were excluded from the analyses when they 1) reported mean/median Body Mass Index (BMI) values instead of weight-status categories, 2) diagnosed overweight/obesity with different criteria from those of the IOTF, 3) reported a combined weight status for both sexes, 4) used children aged below 10 years old in the reported prevalence, 5) consisted of overlapping studies, 6) used samples recruited from hospitals (i.e. with a possible or diagnosed pathological condition), 7) used non-healthy participants and 8) collected data prior to the year 2000.

\section{Quality of the retrieved studies and sensitivity analysis}

Specific protocol/sample characteristics for adolescent studies ${ }^{11}$ and the criteria based on the Newcastle-Ottawa Scale ${ }^{12}$ for nonrandomized research were adopted and applied in order to single out the studies of good quality. ${ }^{13}$ Studies of good quality were identified as having a sample larger than 500 adolescents, reporting a response rate greater than $70 \%$ and having weight and height measured by researchers instead of being reported by the participants. Studies that did not fulfill these criteria were considered of lower quality.

Sensitivity analyses were performed by removing the studies of lower quality in order to evaluate if the results were statistically significant compared to the total of the retrieved studies. Publication bias was evaluated by using funnel plots. Heterogeneity was determined by the $\mathrm{I}^{2}$ index and when $\mathrm{I}^{2}$ was smaller than $75 \%$, heterogeneity was considered low and subsequently a fixed model meta-analysis was performed, as suggested by Higgins and his associates. ${ }^{14}$

\section{Statistical analyses}

Several studies have used meta-analyses in order to accurately calculate the prevalence of a disease as recorded in the literature, ${ }^{13,15,16}$ and recently one study used the same methodology to accurately determine the prevalence of obesity among Greek children. ${ }^{13}$ Analyses were performed with Comprehensive MetaAnalysis software V2.0 (Biostat Inc, Englewood, NJ, USA). Significance was set at $\mathrm{p} \leq 0.05$. Differences in the prevalence of overweight and obesity between the 
two sexes were assessed with tests for two proportions (MiniTab ${ }^{\circledast}$ version 14.1 Minitab Inc., State College, PA, USA).

\section{RESULTS}

\section{Extraction process}

The performed searches retrieved a total of 31 studies but only 8 fulfilled the inclusion criteria. The flow chart of the selection process is depicted in Figure 1, carried out according to the PRISMA statement guidelines for systematic reviews and meta-analyses. ${ }^{17}$ A total of nine full-text articles were excluded for grouping both sexes together $(n=2)$, reporting mean BMI only $(n=2)$, being reviews $(n=2)$, failing to report prevalence of each weight status category $(n=2)$ and for using a children-only sample $(n=1)$.

The results concerning the weight status of Cypriot adolescents according to the selected eight crosssectional studies are presented in Table 1. Five studies involved original research published in peer review journals and three consisted of university theses. ${ }^{21-23}$ The majority of studies involved national representative samples, except for one that used a sample recruited from the city of Limassol only. ${ }^{24}$ Further details were provided by the authors of three studies in order to exclude participants aged under 10 years old $^{20}$ and in order to separate between overweight and obese adolescents. ${ }^{20,24,25}$ Only two studies determined the prevalence of underweight, ${ }^{21,25}$ a number considered inadequate for performing meta-analyses. The prevalence of underweight ranged between 3.1-5.2\% in the boys and 5.7-12.9\% in the girls. The proportion of obese adolescents exhibited great variability, ranging between $5.3-16.9 \%$ in the boys and $4.5-11.5 \%$ in the girls, whereas overweight was reported to range between $17.1-31.6 \%$ and $12.1-27.7 \%$ in boys and girls, respectively. One study used a boys-only sample. ${ }^{23}$

Only three studies fulfilled the quality criteria set for the sensitivity analyses. ${ }^{18-20}$

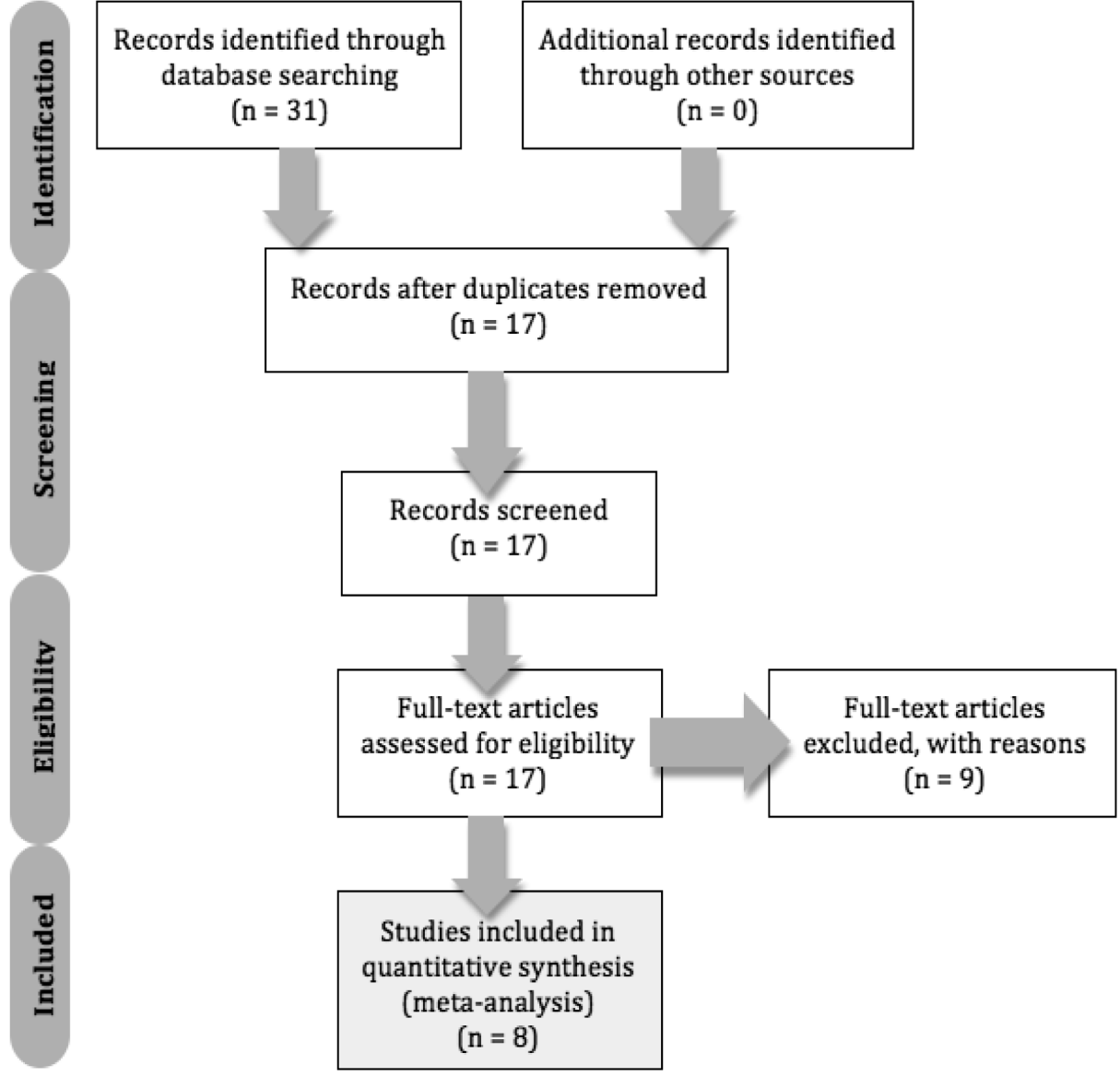

Figure 1. Flow chart of the selection of studies used in the analyses according to the PRISMA statement. ${ }^{17}$ 
Table 1. Studies assessing the weight status of Cypriot adolescents (2000-2010)

\begin{tabular}{|c|c|c|c|c|c|c|c|c|c|c|c|c|c|}
\hline \multirow{2}{*}{$\begin{array}{l}\text { Data } \\
\text { Collection } \\
\text { (year) }\end{array}$} & \multirow{2}{*}{$\begin{array}{c}\text { Study } \\
\text { Sample }\end{array}$} & \multirow[b]{2}{*}{ References } & \multirow{2}{*}{$\begin{array}{c}\text { Total } \\
\text { Sample } \\
\text { Size (n) }\end{array}$} & \multirow{2}{*}{$\begin{array}{l}\text { RR } \\
(\%)\end{array}$} & \multirow{2}{*}{$\begin{array}{c}\text { Age } \\
\text { (years) }\end{array}$} & \multicolumn{4}{|c|}{ Boys } & \multicolumn{4}{|c|}{ Girls } \\
\hline & & & & & & $\mathbf{n}$ & $\% \mathrm{UW}$ & $\% \mathrm{OW}$ & $\% \mathrm{OB}$ & $\mathbf{n}$ & $\% \mathrm{UW}$ & $\% \mathrm{OW}$ & $\% 0 \mathrm{OB}$ \\
\hline 2000 & National $^{1}$ & Savva et al ${ }^{18}$ & $2,600^{3}$ & $94.9 \%$ & $10-18$ & 835 & - & 21.3 & 7.1 & 859 & - & 16.5 & 4.5 \\
\hline 2003 & National $^{1}$ & Savva et al ${ }^{19}$ & $18,792^{3,4}$ & $75.0 \%$ & 11 & 774 & - & 21.4 & 8.8 & 751 & - & 21.8 & 7.0 \\
\hline 2005 & National $^{1}$ & Lazarou et $\mathrm{al}^{20}$ & $682^{3}$ & $91.2 \%$ & $10-14$ & 336 & - & 19.0 & 11.6 & 346 & - & 18.5 & 8.4 \\
\hline 2005 & National & Christaki $^{21}$ & 1,365 & $69.0 \%$ & 11 & 466 & 3.1 & 19.1 & 16.9 & 476 & 5.7 & 15.9 & 11.5 \\
\hline 2006 & National & Lavithi $^{22}$ & 1,500 & $56.9 \%$ & $12-17$ & 373 & - & 24.7 & 5.3 & 481 & - & 12.1 & 2.8 \\
\hline 2007 & National & Photiou $^{23}$ & $4,271^{3}$ & NR & $10-18$ & 2,954 & - & 17.1 & 13.2 & - & - & - & - \\
\hline 2007 & Limassol & Loucaides \& Jago ${ }^{24}$ & 247 & $95.5 \%$ & $10-12$ & 117 & - & 31.6 & 8.5 & 119 & - & 27.7 & 5.0 \\
\hline 2008 & National $^{2}$ & Loucaides et $\mathrm{al}^{25}$ & 1,966 & $91.6 \%$ & $12-16$ & 946 & 5.2 & 21.8 & 7.7 & 854 & 12.9 & 13.8 & 4.8 \\
\hline
\end{tabular}

${ }^{1}$ Studies used in the sensitivity analyses (quality studies), ${ }^{2}$ For the majority of the participants, weight and height were reported, not measured, ${ }^{3}$ The total sample also included participants aged below 10 years old, ${ }^{4}$ The study was cross-sectional at two time points and only the second sample, recruited after the year 2000, was used in the review. RR: Response Rate; NR: not reported; UW: underweight; OW: overweight; OB: obese.

\section{Prevalence of overweight, obesity and combined prevalence in the total sample}

In the total sample (boys and girls grouped together), $18.4 \%$ were considered overweight [95\%CI:17.6$19.2 \%, \mathrm{Q}=21.3, \mathrm{p} \leq 0.001, \mathrm{I}^{2}=67.2 \%$ ] according to the pooled analysis and $19.8 \%$ according to the sensitivity analysis [95\% CI:18.4-21.2\%, Q $=3.4, \mathrm{p} \leq 0.001$, $\left.\mathrm{I}^{2}=40.3 \%\right]$. As far as obesity is concerned, random effects models (REMs) revealed that $8.5 \%$ of adolescents in Cyprus were obese during the years 2000-2010 as calculated by the pooled analysis [95\% CI:6-11\%, $\mathrm{Q}=7.1, \mathrm{p} \leq 0.001, \mathrm{I}^{2}=1.8 \%$ ], compared to the $7.7 \%$ produced by the sensitivity analysis [95\% CI:5.5-9.9\%, $\left.\mathrm{Q}=2.2, \mathrm{p} \leq 0.001, \mathrm{I}^{2}=9.5 \%\right]$. The pooled combined prevalence of overweight and obesity was $27.8 \%$ in the total sample calculated with the use of a REM [95\% CI:25.1-30.5\%, Q =8.6, $\mathrm{p} \leq 0.001, \mathrm{I}^{2}=18.7 \%$ ] and the respective one calculated by the sensitivity analysis reached 27.1\% [95\%CI:25.4-28.7\%, Q=7.7, $\mathrm{p} \leq 0.001, \mathrm{I}^{2}=73.9 \%$ ].

\section{Prevalence of overweight in each sex}

Table 2 shows the prevalence of overweight, obesity and the combined prevalence for each sex. Both the pooled and the sensitivity analyses retrieved a similar prevalence of overweight in boys. More precisely, the pooled analysis revealed that $19.3 \%$ of adolescent boys were overweight [95\% CI:18.3-20.3\%, Q =24.3, $\left.\mathrm{p} \leq 0.001, \mathrm{I}^{2}=71.2 \%\right]$ and the sensitivity analysis produced a prevalence of $20.9 \%$ [95\%CI:18.9-20.3\%,
$\left.\mathrm{Q}=0.7, \mathrm{p} \leq 0.001, \mathrm{I}^{2}=0 \%\right]$. In girls, the pooled prevalence of overweight was calculated with a REM due to heterogeneity as indicated by $\mathrm{I}^{2}$. Thus, $17.1 \%$ of girls were considered overweight [95\% CI:14.2-20\%, $\mathrm{Q}=7.8, \mathrm{p} \leq 0.001, \mathrm{I}^{2}=23.7 \%$ ], a prevalence akin to the one produced by the sensitivity analysis of $18.6 \%$ [95\%CI:16.7-20.5\%, Q =5.8, $\mathrm{p} \leq 0.001, \mathrm{I}^{2}=65.7 \%$ ]. No statistically significant difference was observed between the prevalence of overweight calculated by the pooled and sensitivity analyses in either sex.

\section{Prevalence of obesity in each sex}

When the pooled obesity data were analyzed, the studies showed great heterogeneity $\left(\mathrm{I}^{2}>75 \%\right)$ and, subsequently, REMs were performed. The pooled calculated rate of obesity among boys was $9.8 \%$ [95\%CI:7.3-12.3\%, Q=7.4, $\left.\mathrm{p} \leq 0.001, \mathrm{I}^{2}=4.8 \%\right]$ and $6.1 \%$ among adolescent girls [95\% CI:4.2-8\%, $\mathrm{Q}=7.7$, $\left.\mathrm{p} \leq 0.001, \mathrm{I}^{2}=22.4 \%\right]$. The sensitivity analyses revealed that $8.3 \%$ of boys [95\% CI:7-9.5\%, Q $=5.2, \mathrm{p} \leq 0.001$, $\left.\mathrm{I}^{2}=61.3 \%\right]$ and $5.8 \%$ of girls [95\%CI:4.7-6.9\%, $\mathrm{Q}=7.4$, $\left.\mathrm{p} \leq 0.001, \mathrm{I}^{2}=73.1 \%\right]$, respectively, were obese during the examined time frame.

\section{Combined prevalence of overweight and obesity in each sex}

As far as the combined prevalence of overweight and obesity is concerned, the pooled produced prevalence for boys was 30.4\% [95\%CI:29.1-31.7\%, Q =8.4, $\left.\mathrm{p} \leq 0.001, \mathrm{I}^{2}=16.8 \%\right]$ and the ratio calculated by the 
Table 2. Aggregated results of the pooled and sensitivity analyses of studies assessing the prevalence of overweight and obesity in Cypriot adolescents (2000-2010)

\begin{tabular}{|c|c|c|c|c|c|c|c|c|c|c|c|c|c|c|c|c|c|}
\hline \multirow[b]{3}{*}{ Analyses } & \multirow{3}{*}{$\begin{array}{l}\text { Weight } \\
\text { Status }\end{array}$} & \multicolumn{6}{|c|}{ Boys } & \multicolumn{6}{|c|}{ Girls } & \multirow{2}{*}{$\underset{\text { between }}{p}$} & \multirow[b]{3}{*}{ OR } & \multirow{2}{*}{\multicolumn{2}{|c|}{$95 \%$ CI }} \\
\hline & & & Total & $95 \%$ & $6 \mathrm{CI}$ & & & & tal & $95 \%$ & $6 \mathrm{CI}$ & & & & & & \\
\hline & & $\%$ & $\mathbf{n}$ & Lower & Upper & Q & $I^{2}$ & $\%$ & $\mathbf{n}$ & Lower & Upper & Q & $\mathbf{I}^{2}$ & sexes & & Lower & Upper \\
\hline \multirow{3}{*}{$\begin{array}{l}\frac{0}{0} \\
\frac{0}{0} \\
\varrho\end{array}$} & Obesity & 9.8 & 6,081 & 7.3 & 12.3 & 7.4 & 4.8 & $6.1^{*}$ & 3,886 & 4.2 & 8.0 & 7.7 & 22.4 & 0.001 & 1.7 & 1.4 & 2.0 \\
\hline & Overweight & 19.3 & 6,081 & 18.3 & 20.3 & 24.3 & 71.2 & 17.1 & 3,886 & 14.2 & 20.0 & 7.8 & 23.7 & 0.001 & 1.3 & 1.2 & 1.4 \\
\hline & $\begin{array}{l}\text { Overweight } \\
\text { including } \\
\text { obesity }\end{array}$ & 30.4 & 6,081 & 29.1 & 31.7 & 8.4 & 16.8 & 23.6 & 3,886 & 19.3 & 27.9 & 6.7 & 9.8 & 0.001 & 1.5 & 1.3 & 1.6 \\
\hline \multirow{3}{*}{ 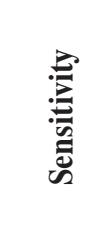 } & Obesity & 8.3 & 1,945 & 7.0 & 9.5 & 5.2 & 61.3 & 5.8 & 1,956 & 4.7 & 6.9 & 7.4 & 73.1 & 0.032 & 1.2 & 1.1 & 1.4 \\
\hline & Overweight & 20.9 & 1,945 & 18.9 & 20.3 & 0.7 & 0.0 & 18.6 & 1,956 & 16.7 & 20.5 & 5.8 & 65.7 & 0.116 & 1.1 & 1.0 & 1.3 \\
\hline & $\begin{array}{l}\text { Overweight } \\
\text { including } \\
\text { obesity }\end{array}$ & 29.5 & 1,945 & 27.1 & 31.9 & 0.7 & 0.0 & 25.4 & 1,956 & 20.1 & 20.6 & 1.6 & 0.0 & 0.001 & 1.2 & 1.1 & 1.4 \\
\hline
\end{tabular}

*Significantly different compared to the sensitivity analysis ( $\leq 0.001)$. CI: Confidence Intervals; OR: Odds Ratios for boys; Q: Cochrane's Q statistic.

sensitivity analysis reached 29.5\% [95\% CI:27.1-31.9\%, $\mathrm{Q}=0.7, \mathrm{p} \leq 0.001, \mathrm{I}^{2}=0 \%$ ]. In girls, REMs were used in the analyses and the combined prevalence of overweight and obesity was calculated as a pooled $23.6 \%$ [95\% CI:19.3-27.9\%, Q =6.7, $\mathrm{p} \leq 0.001, \mathrm{I}^{2}=9.8 \%$ ], whereas the sensitivity analysis revealed $25.4 \%$ of combined prevalence [95\% CI:20.1-20.6\%, Q $=1.6$, $\mathrm{p} \leq 0.001, \mathrm{I}^{2}=0 \%$ ].

\section{Comparisons between sexes}

Between sexes, boys demonstrated a higher prevalence of obesity and overweight ( $\mathrm{p} \leq 0.001$ for both) compared to girls. According to the pooled analysis, the odds ratio (OR) for overweight in adolescent boys compared to girls was 1.3 (CI:1.2-1.4), whereas for obesity OR was 1.7 (CI:1.4-2) ( $\mathrm{p} \leq 0.001$ for both). The boys exhibited 1.5 greater odds of being either overweight or obese during adolescence compared to girls (OR:1.5, CI:1.3-1.6). The sensitivity analyses revealed lower odds for all examined parameters (OR obesity:1.4, CI:1.1-1.8; OR overweight:1.1, CI:1-1.3; OR overweight/obesity:1.2, CI:1.1-1.4).

\section{Trend of the combined prevalence of overweight including obesity during the years 2000-2010}

Pooled cumulative analyses on the prevalence of overweight including obesity in each sex are presented in Table 3, using the eight and seven retrieved stud- ies with boys' and girls' samples, respectively. Both sexes demonstrated a slight increase apparent until the year 2005. In boys, stabilization in the prevalence of overweight/obesity was observed from the year 2005 and onwards, whereas girls experienced a slight non-linear decrease.

\section{DISCUSSION}

According to the present analyses, a total of $8.5 \%$ of Cypriot adolescents were obese during the years 2000-2010, $18.4 \%$ were overweight and $27.8 \%$ were either overweight or obese. Between sexes, $6.4 \%$ of girls and $9.8 \%$ of boys were considered obese during adolescence, whereas $17.1 \%$ and $19.3 \%$, respectively, from each sex were considered overweight. Overall, approximately $1 / 4$ of girls and $1 / 3$ of boys were either overweight or obese. Data on the prevalence of underweight are limited and research reporting the prevalence of central obesity is scarce. It is difficult to define the time point when the prevalence of adolescent overweight started rising in the island, since there are no available epidemiological studies prior to the year 2000. However, for both sexes the combined prevalence of overweight/obesity culminated during the year 2005. From then and onwards the boys stabilized their prevalence and the girls demonstrated an irregular decrease. 
Table 3. Cumulative prevalence of overweight/obesity in Cypriot adolescent boys (a) and girls (b) (2000-2010)

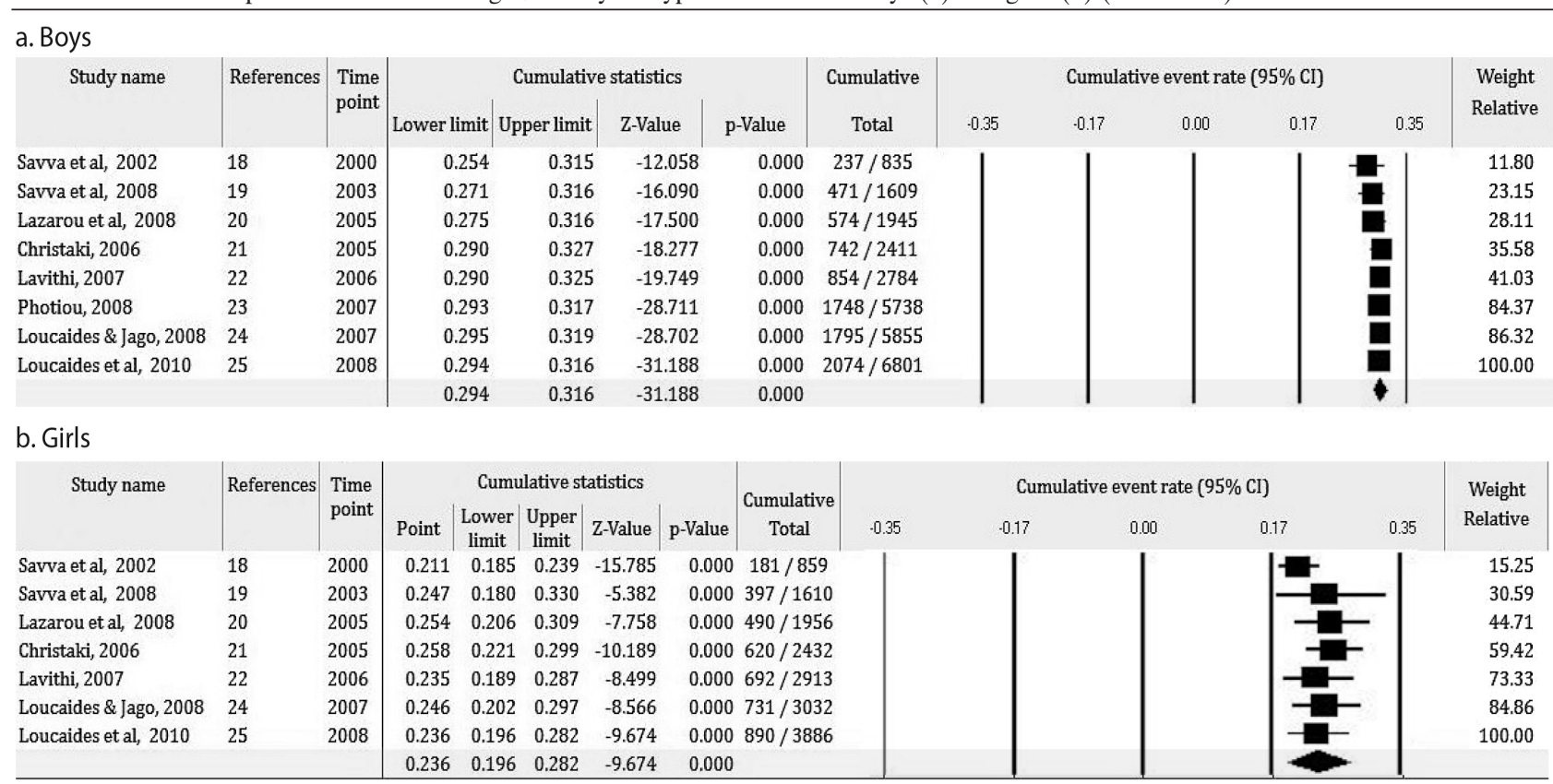

In Europe, the prevalence of childhood and adolescent overweight appears to form a gradient, with the countries situated on the lower latitudes presenting the highest rates observed. ${ }^{26}$ Cyprus is geographically situated at the lowest latitude of the continent, thus the increased rates of overweight and obesity observed in the present analysis provide further verification of the gradient theory. It has been suggested that the high prevalence of overweight observed in Southern Europe might be the result of the hot climate, as warm environments are associated with reduced thermogenesis and increased sedentary behavior due to the discomfort experienced when exercising in high temperatures. ${ }^{26}$ In confirmation of this obesogenic climate effect theory, research in Cyprus has linked childhood obesity with increased screen time and other sedentary behaviors. ${ }^{27}$

Several factors may contribute to the high prevalence of overweight, including a genetic predisposition, which is confirmed by studies on Cypriot adults, half of whom appear to be overweight/obese..$^{20}$ Unhealthy dietary choices constitute another potential explanation for the high prevalence of overweight/obesity, as the majority of Cypriot children and adolescents follow a diet of low quality. ${ }^{28}$ Additionally, bilateral parental obesity has been shown to increase the chances of offspring obesity by 18.1 times in adolescent Cypriot boys. ${ }^{18}$

Another factor contributing to the obesogenity of an environment is urbanization. ${ }^{26}$ Living in urban Cyprus has been found to affect dietary choices during adolescence, ${ }^{29}$ but data concerning the prevalence of overweight appear conflicting. Loucaides ${ }^{22}$ reported that rural Cypriot girls exercise less and Christaki ${ }^{21}$ added another finding by demonstrating higher mean BMI in rural Cypriot adolescents compared to the urban inhabitants of Larnaka and Limassol cities. Savva and his associates ${ }^{19}$ compared the relative increase in the prevalence of obesity during a 5-year period and found a substantially higher increase in rural adolescents $(35.9 \%)$ compared with those living in urban areas of Cyprus (8.7\%). However, the CYKIDS study failed to associate obesity with urbanization in school children..$^{20}$ Therefore, the exact effect of urbanization on adolescent obesity does not appear to be clear-cut, as suggested by other researchers. ${ }^{30}$ Differing definitions as to what constitutes urban/rural in each country and differences in the physical and social environment of each study's location further cloud the obesity-urbanization issue..$^{30,31}$

Today, the number of studies assessing excess in body weight according to the IOTF criteria is 
constantly increasing, ${ }^{32}$ but these do not necessarily provide quality data. Although the WHO has aptly put adolescent health in the spotlight, ${ }^{8}$ data on the prevalence of overweight/obesity during adolescence in Europe appear scattered and heterogeneous. ${ }^{32}$ This creates the need for available high-quality data objectively measuring overweight/obesity in national representative samples of European adolescents. ${ }^{32}$ In addition, the need for quality assessment of all studies assessing overweight and obesity is universal, ${ }^{26,30,32}$ as low quality studies either over- or under-estimate the actual prevalence. An example of underestimation of the prevalence of obesity is manifest in the difference observed between pooled and sensitivity analysis performed in the girls' data from the results herein. This problem can be addressed by performing systematic reviews and meta-analyses on the prevalence of overweight in each country as well as in the whole continent.

Both sexes demonstrated a slight increase in the prevalence of overweight/obesity that peaked during the year 2005. As data prior to the year 2000 do not exist, it is possible that the first studies conducted between the years 2000-2005 alarmed both the public health sector and the parents and this augmentative trend was halted. In addition, the characterization of obesity as an epidemic also took place early during the 2000's. ${ }^{33}$ These warnings produced immediate policy options for responding to the growing obesity challenge from the Cyprus Institute of Child Health ${ }^{6}$ and triggered the initiation of several communityoriented interventions aiming to promote the adoption of a healthy obesity-preventing lifestyle, like the multi-centered European IDEFICS consortium, ${ }^{5}$ or the Cypriot initiative of adopting a $15 \mathrm{~min}$ work-out every morning for school staff and pupils. ${ }^{34}$ According to the cumulative analyses, the combined prevalence of overweight and obesity ceased spiraling during the year 2005. Since then, a stabilization in prevalence has been observed among boys and a slight decrease in girls; however, the overall prevalence is still elevated and further interventions are needed in order to combat the disease. During the year 2005, the Cypriot Ministry of Health joined the European Network for Health Education in Schools and since the year 2006 structured protocols of health education, including nutrition, have been adopted adopted in schools throughout the country. Although we do not have evidence of a direct impact of these health education protocols, it is highly likely that the efforts to ameliorate nutritional knowledge and correct the lack of physical inactivity in adolescents have had an effect on halting the increasing prevalence of overweight.

The present review also identified heterogeneity in several aspects of the available data and lack of data concerning the prevalence of abdominal obesity and underweight, as reported by others. ${ }^{30,32}$ In fact, the majority of studies report mean waist circumference measurements instead of the prevalence of abdominal obesity and, as far as underweight is concerned, researchers appear to underestimate the increased prevalence observed especially in girls, and in their majority do not distinguish between underweight and normal body weight adolescents. Between pooled and sensitivity analyses, a higher proportion of adolescents were considered obese in the latter, showing a compromised accuracy in the results published by lower quality studies and adding more emphasis to the need for high quality studies to be performed. Heterogeneity was also observed in the age-range defining adolescence and this produces problems in comparing data. According to $\mathrm{WHO},{ }^{8}$ the age spectrum for adolescence ranges between 10 and 19 years; however, several researchers use convenient samples with either wider or smaller age-ranges that tend to either under- or over-estimate the actual prevalence of overweight in the adolescent population. In samples recruited from hospitals, adolescence is often defined according to the Marshall and Tanner stages, ${ }^{35,36}$ a classification often conflicting with the proposed WHO age-range.

Compendiously, the present study demonstrated high rates of overweight and obesity among Cypriot adolescents during the decade 2000-2010. Overall, this study shows that we are in need of quality studies also calculating the prevalence of underweight and abdominal obesity as such data are either missing or limited. Additionally, school-based structured nutrition and physical activity education programs including parental involvement should be continued in order to ensure a healthier adolescent and future adult population. Due to the lack of similar studies across Europe, comparisons of the present findings are not feasible. In addition, it is suggested that systematic 
reviews and meta-analyses should be performed in more European countries in order to obtain objective data on the prevalence of overweight/obesity among different age groups.

\section{REFERENCES}

1. Lobstein T, Baur L, Uauy R, 2004 Obesity in children and young people: a crisis in public health. Obes Rev 5: 4-104.

2. Fonseca H, Matos MG, Guerra A, Pedro JG, 2009 Are overweight adolescents at higher risk of engaging in unhealthy weight-control behaviours? Acta Paediatr 98: 847-852.

3. Cavazos-Rehg PA, Krauss MJ, Spitznagel EL, Schootman M, Cottler LB, Bierut LJ, 2010 Associations between multiple pregnancies and health risk behaviors among U.S. adolescents. J Adolesc Health 47: 600-603.

4. Burt Solorzano CM, McCartney CR, 2010 Obesity and the pubertal transition in girls and boys. Reproduction 140: 399-410.

5. De Henauw S, Verbestel V, Mårild S, 2011 The IDEFICS community-oriented intervention programme: a new model for childhood obesity prevention in Europe? Int J Obes (London) 35: Suppl 1: 16-23.

6. Savva SC, Chadjioannou M, Tornaritis MJ, 2007 Policy options for responding to the growing challenge from obesity: Cyprus national findings. Obes Rev 8S2: 37-45.

7. Young SS, Bang H, 2004 The file-drawer problem, revisited. Science 306: 1133-1134.

8. World Health Organization, 2002 Adolescent friendly health services. An agenda for change. [16 April 2014]: Available from: http://whqlibdoc.who.int/hq/2003/ WHO_FCH_CAH_02.14.pdf

9. Cole TJ, Bellizzi MC, Flegal KM, Dietz WH, 2000 Establishing a standard definition for child overweight and obesity worldwide: international survey. BMJ 320: 1240.

10. Cole TJ, Flegal KM, Nicholls D, Jackson AA, 2007 Body mass index cut offs to define thinness in children and adolescents: international survey. BMJ 335: 194-202.

11. Lien N, Henriksen HB, Nymoen LL, Wind M, Klepp KI, 2010 Availability of data assessing the prevalence and trends of overweight and obesity among European adolescents. Public Health Nutr 13: 1680-1687.

12. Wells GA, Shea B, O'Connell D, et al, The NewcastleOttawa Scale (NOS) for assessing the quality of nonrandomized studies in meta-analyses. [16 April 2014]: Available from: http://www.ohri.ca/programs/clinical_epidemiology/oxford.htm

13. Kotanidou EP, Grammatikopoulou MG, Spiliotis BE, Kanaka-Gantenbein C, Tsigga M, Galli-Tsinopoulou A, 2013 Ten-Year obesity and overweight prevalence in Greek children: A systematic review and meta-analysis of 2001-2010 data. Hormones (Athens) 12: 537-549.

14. Higgins JPT, Thompson SG, Deeks JJ, Altman DG,
2003 Measuring inconsistency in meta-analyses. BMJ 327: 557-560.

15. Chen JJ, Yu CB, Du WB, Li LJ, 2011 Prevalence of hepatitis $\mathrm{B}$ and $\mathrm{C}$ in HIV-infected patients: a meta-analysis. Hepatobiliary Pancreat Dis Int 10: 122-127.

16. Tsigga M, Grammatikopoulou MG, 2012 Assessing the silent epidemic of malnutrition in Palestinian preschool children. J Epidemiol Glob Health 2: 181-191.

17. Moher D, Liberati A, Tetzlaff J, Altman DG, The PRISMA Group, 2009 Preferred Reporting Items for Systematic Reviews and Meta-Analyses: The PRISMA Statement. PLoS Med 6: e1000097.

18. Savva SC, Kourides Y, Tornaritis M, Epiphaniou-Savva M, Chadjigeorgiou C, Kafatos A, 2002 Obesity in children and adolescents in Cyprus. Prevalence and predisposing factors. Int J Obes Relat Metab Disord 26: 1036-1045.

19. Savva SC, Tornaritis MJ, Chadjigeorgiou C, Kourides YA, Siamounki M, Kafatos A, 2008 Prevalence of overweight and obesity among 11-year-old children in Cyprus, 1997-2003. Int J Pediatr Obes 3: 186-192.

20. Lazarou C, Panagiotakos DB, Panayiotou G, Matalas AL, 2008 Overweight and obesity in preadolescent children and their parents in Cyprus: prevalence and associated socio-demographic factors - the CYKIDS study. Obes Rev 9: 185-193.

21. Christaki M, 2006 Correlation between childhood obesity and overweight with possible predisposing factors in a Cypriot sample of 11 year-olds. MSc Thesis, Harokopio University, Athens, Greece. http://estia.hua.gr:8080/ dspace/bitstream/123456789/98/1/Metaptychiaki21.pdf

22. Lavithi D, 2007 Assessment of the dietary habits of adolescents aged 12-17 years old from Cyprus. MSc Thesis, Harokopio University, Athens, Greece. http://estia.hua. gr:8080/dspace/bitstream/123456789/368/1/lavithi.pdf

23. Photiou A, 2008 Somatic development and body composition of 6- to 18-year-old boys - The first Cypriot growth study. PhD Thesis, Semmelweis University, Budapest, Hungary.

24. Loucaides CA, Jago R, 2008 Differences in physical activity by gender, weight status and travel mode to school in Cypriot children. Prev Med 47: 107-111.

25. Loucaides CA, Jago R, Theophanous M, 2010 Prevalence and correlates of active travelling to school among adolescents in Cyprus. Cent Eur J Public Health 18: 151-156.

26. Lobstein T, Frelut M-L, 2003 Prevalence of overweight among children in Europe. Obes Rev 4: 195-200.

27. Lazarou C, Soteriades ES, 2010 Children's physical activity, TV watching and obesity in Cyprus: the CYKIDS study. Eur J Public Health 20: 70-77.

28. Lazarou C, Panagiotakos DB, Kouta C, Matalas AL, 2009 Dietary and other lifestyle characteristics of Cypriot school children: results from the nationwide CYKIDS study. BMC Public Health 9: 147.

29. Lazarou C, Kalavana T, 2009 Urbanization influences dietary habits of Cypriot children: the CYKIDS study. 
Int J Public Health 54: 69-77.

30. Mirkopoulou D, Grammatikopoulou MG, Gerothanasi K, Tagka A, Stylianou C, Hassapidou M, 2010 Metabolic indices, energy and macronutrient intake according to weight status in a rural sample of 17-year-old adolescents. Rural Remote Health 10: 1513.

31. Hodgkin E, Hamlin MJ, Ross JJ, Peters F, 2010 Obesity, energy intake and physical activity in rural and urban New Zealand children. Rural Remote Health 10: 1336.

32. Lien N, Henriksen HB, Nymoen LL, Wind M, Klepp KI, 2010 Availability of data assessing the prevalence and trends of overweight and obesity among European adolescents. Public Health Nutr 13: 1680-1687.

33. James PT, Leach R, Kalamara E, Shayeghi M, 2001 The Worldwide Obesity Epidemic. Obes Res 9: Suppl 4: 228-233.

34. Lobstein T, 2006 Comment: Preventing child obesity an art and a science. Obes Rev 7: 1-5.

35. Marshall WA, Tanner JM, 1969 Variations in pattern of pubertal changes in girls. Arch Dis Child 44: 291-303.

36. Marshall WA, Tanner JM, 1970 Variations in pattern of pubertal changes in boys. Arch Dis Child 45: 13-23. 


\title{
A SOX3 (Xq26.3-27.3) duplication in a boy with growth hormone deficiency, ocular dyspraxia, and intellectual disability: A long-term follow-up and literature review
}

\author{
Stefano Stagi, ${ }^{1}$ Elisabetta Lapi, ${ }^{2}$ Marilena Pantaleo, ${ }^{2}$ Giovanna Traficante, ${ }^{2}$ \\ Sabrina Giglio, ${ }^{2}$ Salvatore Seminara, ${ }^{1}$ Maurizio de Martino ${ }^{1}$ \\ ${ }^{1}$ Paediatric Endocrinology Unit, Department of Health Sciences, ${ }^{2}$ Genetics and Molecular Medicine Unit; Anna Meyer \\ Children's University Hospital, Florence, Italy
}

\begin{abstract}
OBJECTIVE: SOX3 is located on the long arm of the $\mathrm{X}$ chromosome (Xq27.1) and both the under- and over-expression of this gene have been reported in cases of hypopituitarism with or without intellectual disabilities. Nevertheless, only a few cases have as yet been extensively described. DESIGN: A 3-year 11 month-old male was brought in for growth failure (height -2.4 SDS). The patient was born at term of a second uneventful pregnancy by caesarean section for podalic presentation: the birth weight $(0.1$ SDS), length $(0.4$ SDS), and head circumference (-0.3 SDS) were normal. Neurodevelopmental delays and ocular motor dyspraxia had been noted since 6 months of age. The endocrinological evaluation showed a very low IGF-I concentration $(44 \mu \mathrm{g} / \mathrm{L})$. The thyroid hormone level was normal and coeliac disease markers were negative. Bone age was considerably delayed. Target height was normal (0.5 SDS). RESULTS: Growth hormone stimulation tests were compatible with a classic GHD, while a brain MRI disclosed a pituitary hypoplasia with ectopic neurohypophysis. rhGH treatment was then begun and the auxological follow-up showed a good response. At the age of $9 \mathrm{yrs}$, the height was $0.3 \mathrm{SDS}$, the weight was 0.1 SDS, and the pubertal evaluation was PH1 AH1 T2 ml bilaterally. Due to the presence of neuromotor delays and MRI abnormalities, a genetic evaluation was conducted and an array-CGH of the patient's DNA discovered an Xq26.3-27.3 duplication comprising the SOX3 gene. CONCLUSIONS: SOX3 involvement should be considered in a male with short stature due to GH deficiency associated with intellectual disability.
\end{abstract}

Key words: Dyspraxia, Growth Hormone, Growth Hormone Deficiency, Intellectual Disability Short stature, $\mathrm{SOX} 3$

Address for correspondence:

Stefano Stagi, MD, Health Sciences Department, Anna Meyer Children's University Hospital, Viale Pieraccini 24,

50139 Florence, Italy, Tel.: +39-055-5662622;

Fax: +39-055-4221012; E-mail: stefano.stagi@yahoo.it

Received: 26-01-2014, Accepted: 16-06-2014

\section{INTRODUCTION}

Pituitary gland development and function depend on the sequential temporal and spatial expression of multiple transcription factor genes, such as POU1F1 (POU class 1 homeobox 1; OMIM 173110), PROP1 
(prophet of PIT1; OMIM 601538), HESX1 (homeobox gene expressed in ES cells; OMIM 601802), LHX3 (LIM homeobox gene 3; OMIM 600577), LHX4 (LIM homeobox gene 4; OMIM 602146), SOX3 (SRY-related HMG-box gene 3; OMIM 313430), and OTX2 (orthodenticle homolog 2; OMIM 600037). ${ }^{1-3}$

Congenital hypopituitarism $(\mathrm{CH})$, a defect that is characterised by a deficiency in one or more pituitary hormones and is not rare, may be caused by mutations in any of these genes. ${ }^{4}$

$\mathrm{CH}$ manifests either as an isolated hormone deficiency, the most common being isolated growth hormone deficiency (IGHD) or as multiple pituitary hormone involvement [combined pituitary hormone deficiencies (CPHD)]. ${ }^{1-3}$

The $\mathrm{CH}$ clinical features may be detected in the neonatal period or present later in life. ${ }^{4,5}$ Moreover, hormonal deficits may be associated with extra-pituitary defects affecting organs that are embryologically correlated. ${ }^{4,5}$

SOX3 is a single exon gene located on the long arm of the $\mathrm{X}$ chromosome (Xq27.1). SOX3 is a member of the SOX (SRY-related high mobility group box) family of transcription factors that is expressed in neuroepithelial progenitor and stem cells beginning in the earliest stages of embryogenesis. ${ }^{6,7}$ Sox $/ S O X$ genes have been recognised as key players in the regulation of embryogenesis and nervous system development; they encode transcription factors that act as key regulators in different developmental processes, such as gastrulation, neural induction, specification, and the differentiation of many cell types. ${ }^{8,9}$ SOX3 has also been implicated in the aetiology of a septo-optic dysplasia variant. ${ }^{3}$

The dysfunction of the SOX3 protein disturbs cellular processes that are required for cognitive and pituitary development. ${ }^{10}$ In fact, in human males, both the under- and over-expression of this gene lead to CPHD or IGHD and infundibular hypoplasia, an ectopic/undescended posterior pituitary and abnormalities of the corpus callosum with or without intellectual disability (ID). ${ }^{10-12}$

However, micro-duplications of $S O X 3$ have been identified in only a few patients with IGHD or CPHD, ${ }^{10,13-16}$ frequently accompanied by poor endocrinological ${ }^{13,15,16}$ or clinical ${ }^{10,13}$ data.

\section{CASE REPORT}

The propositus was the second child of young, healthy, non-consanguineous parents of Italian origin. The target height was normal [0.5 standard deviation score 9SDS)]. After a miscarriage, the couple had a son with normal growth and neuropsychological development (the pedigree is illustrated in Figure 1).

The patient was born by caesarean section for podalic presentation at term of the $3^{\text {rd }}$ uneventful pregnancy. The birth weight was $3.030 \mathrm{~kg}(0.1 \mathrm{SDS})$, the length was $52 \mathrm{~cm}(0.4 \mathrm{SDS})$ and the head circumference was $34.8 \mathrm{~cm}$ (-0.3 SDS). The Apgar score was $9^{\mathrm{I}}-10^{\mathrm{V}}$. Genital abnormalities were not observed, nor were hypoglycaemias.

During the first year of life, a mild developmental delay became evident: he sat at 8 months and walked independently at 22 months, while language started at 24 months. Intellectual disability was ascertained at the age of $2 \mathrm{yr}$ and $6 \mathrm{mo}$ : the developmental quotient (DQ) was 65. In the same period, an ophthalmologic examination was carried out: the fundus and visual acuity were normal, but a gaze-evoked horizontal nystagmus and ocular saccadic overshot were observed, leading to a suspicion of a diagnosis of ocular dyspraxia.

At 3 yr and 11 mo old, due to pronounced growth failure (height -2.4 SDS), an endocrinological evaluation of the child was conducted which revealed a very low IGF-I concentration $(44 \mu \mathrm{g} / \mathrm{L})$. An extensive endocrine work-up was performed: free-thyroxin [(FT $\left.{ }_{4}\right) 1.47 \mathrm{ng} / \mathrm{dL}$, n.v. 0.86-2.12 ng/dL], thyroidstimulating hormone [(TSH) $3.38 \mu \mathrm{UI} / \mathrm{dL}$, n.v. 0.4$4.0 \mu \mathrm{UI} / \mathrm{dL}]$, cortisol $(8.23 \mu \mathrm{g} / \mathrm{dL}$, n.v. $5-25 \mu \mathrm{g} / \mathrm{dL})$, adrenocorticotropic hormone [(ACTH) $50 \mathrm{ng} / \mathrm{L}$, n.v. 09-52 ng/L), glucose (72 mg/dL, n.v. 55-110 mg/dL), and prolactin $[(\mathrm{PRL}) 86 \mathrm{mUI} / \mathrm{ml}]$ were in the normal range. The electrolyte, venous blood gas, haemoglobin, total protein, serum albumin, coagulation profile, calcium, phosphorous, vitamin D (25OHD), and parathyroid hormone (PTH) levels were also normal. The anti-tissue transglutaminase (tTG) was negative. Neuro-metabolic tests (plasma aminoacidogram, urine aminoacidogram, acylcarnitine profile analysis, and redox state) yielded normal results. The karyotype was $46, X Y$. A multiplex ligation-dependent probe amplification (MLPA) analysis and fragile $X$ syndrome (FRAXA) testing also returned normal results. 


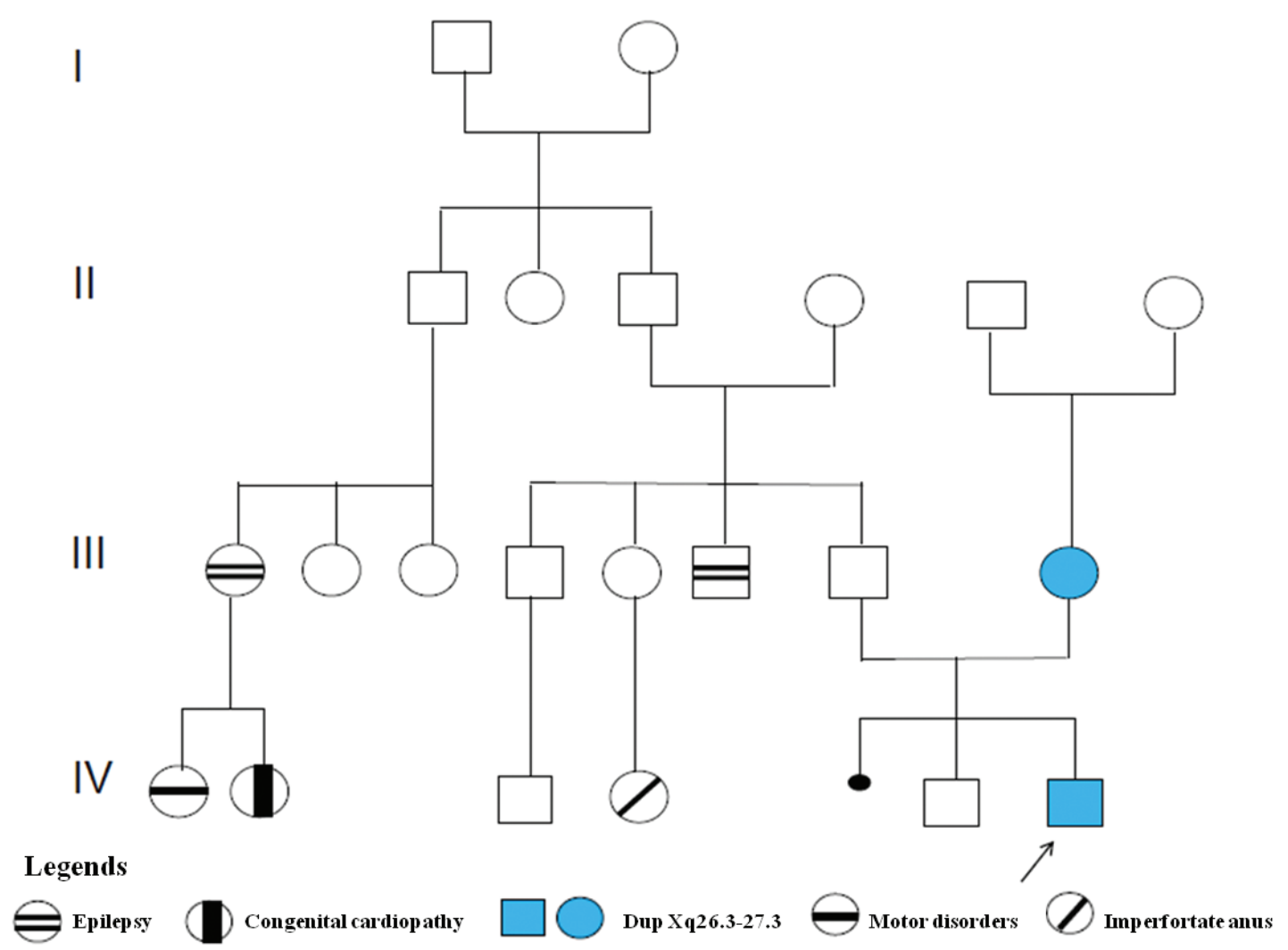

Figure 1. Pedigree of the family that was analysed in this study. Duplications were found in the proband and mother.

Bone age was considerably delayed (2 yr and $1 \mathrm{mo}$ at $3 \mathrm{yr}$ and $11 \mathrm{mo}$ of chronological age). A growth hormone stimulation test disclosed a classic GH deficiency (GH peak after clonidine $2.4 \mathrm{ng} /$ $\mathrm{mL}$; GH peak after arginine $2.1 \mathrm{ng} / \mathrm{mL}$ ). An MRI revealed an anterior pituitary hypoplasia with ectopic neurohypophysis, corpus callosum hypogenesis, and incomplete myelination (Figure 2). The posterior fossa was significantly reduced.

Based on these findings, rhGH treatment was conducted $(0.23 \mathrm{mg} / \mathrm{kg}$ per week subcutaneously). The auxological follow-up showed a strong positive response to the treatment with the standard deviationgrowth velocity (SDS-GV) increasing remarkably during therapy (Figure 3).

At $9 \mathrm{yr}$ and $9 \mathrm{mo}$, the height was 0.3 SDS, the weight was $0.1 \mathrm{SDS}$, and the pubertal evaluation was PH1 AH1 T2 ml bilaterally. Bone age remained considerably delayed ( $6 \mathrm{yr}$ and $1 \mathrm{mo}$ at $9 \mathrm{yr}$ and 9 mo of chronological age). During follow-up, a periodic evaluation of the other adenohypophyseal hormones

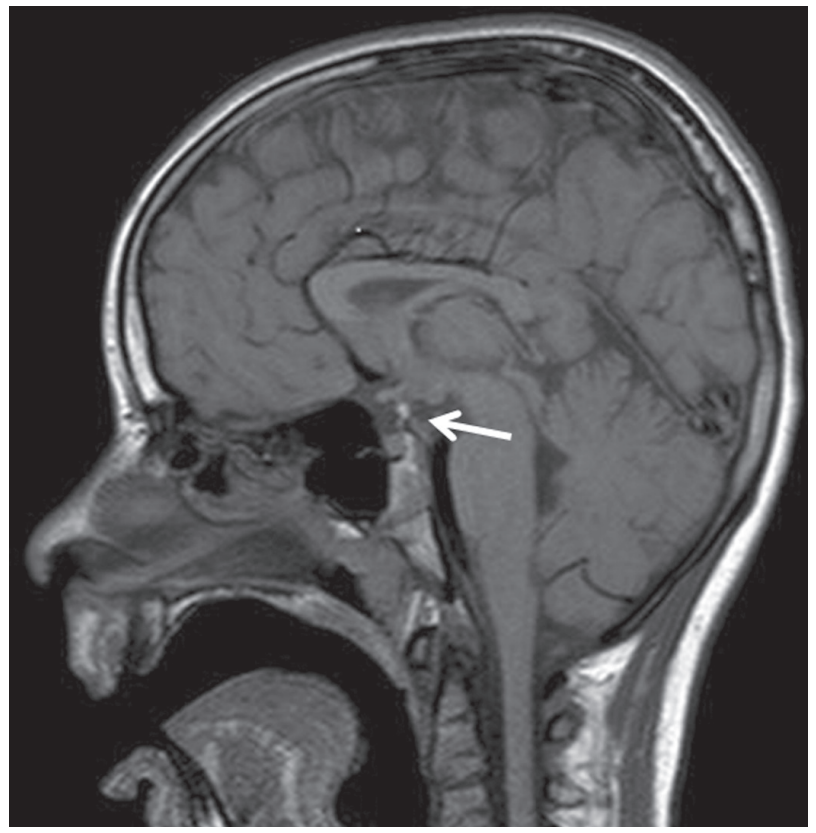

Figure 2. Sagittal MRI scan of the patient with $S O X 3$ duplication, showing pituitary hypoplasia, hypoplasia of the infundibulum, and an undescended/ectopic posterior pituitary. Note the hypogenesis of the corpus callosum. 


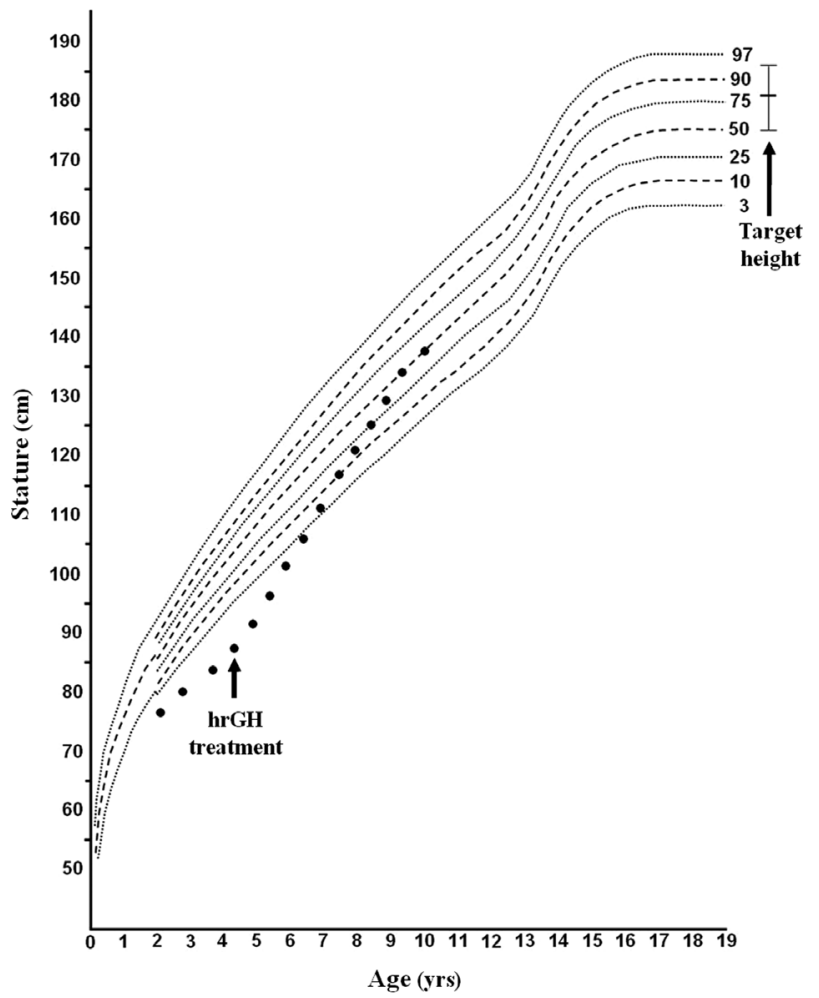

Figure 3. Growth chart of the patient. The arrows indicate the age of onset for growth hormone treatment (black arrow). disclosed deficiency. At $6 \mathrm{yr}$ and 8 mo of age, the $\mathrm{FT}_{4}$ was $1.56 \mathrm{ng} / \mathrm{dL}$, the TSH was $3.01 \mu \mathrm{UI} / \mathrm{dL}$, the cortisol was $11.45 \mu \mathrm{g} / \mathrm{dL}$, the ACTH was $42 \mathrm{ng} / \mathrm{L}$ and the PRL was $69 \mathrm{mUI} / \mathrm{ml}$.

Due to the presence of neuromotor delays and MRI abnormalities, a genetic evaluation was carried out. The patient's DNA was analysed using array CGH (comparative genomic hybridisation). After obtaining informed consent, the genomic DNA was extracted from the leukocytes of the proband, i.e. both his parents and maternal grandparents according to standard procedures. Array CGH was performed using the Agilent 60k platform with a median resolution rate of nearly 100 kilobases $(\mathrm{kb})$. Based on the physical mapping positions that were designated at the March 2006 assembly (NCBI36/ hg18) of the UCSC Genome Browser, this analysis showed a duplication that involved the Xq26.3-27.3 region with an extension between 135,175,703 bp (first duplicated) and 142,971,531 bp (last duplicated) (Figure 4; Table 1): a 7.8 megabase (Mb) duplication was identified in Xq26.3 - 27.3 spanning more than 20 genes, among which the morbid genes were

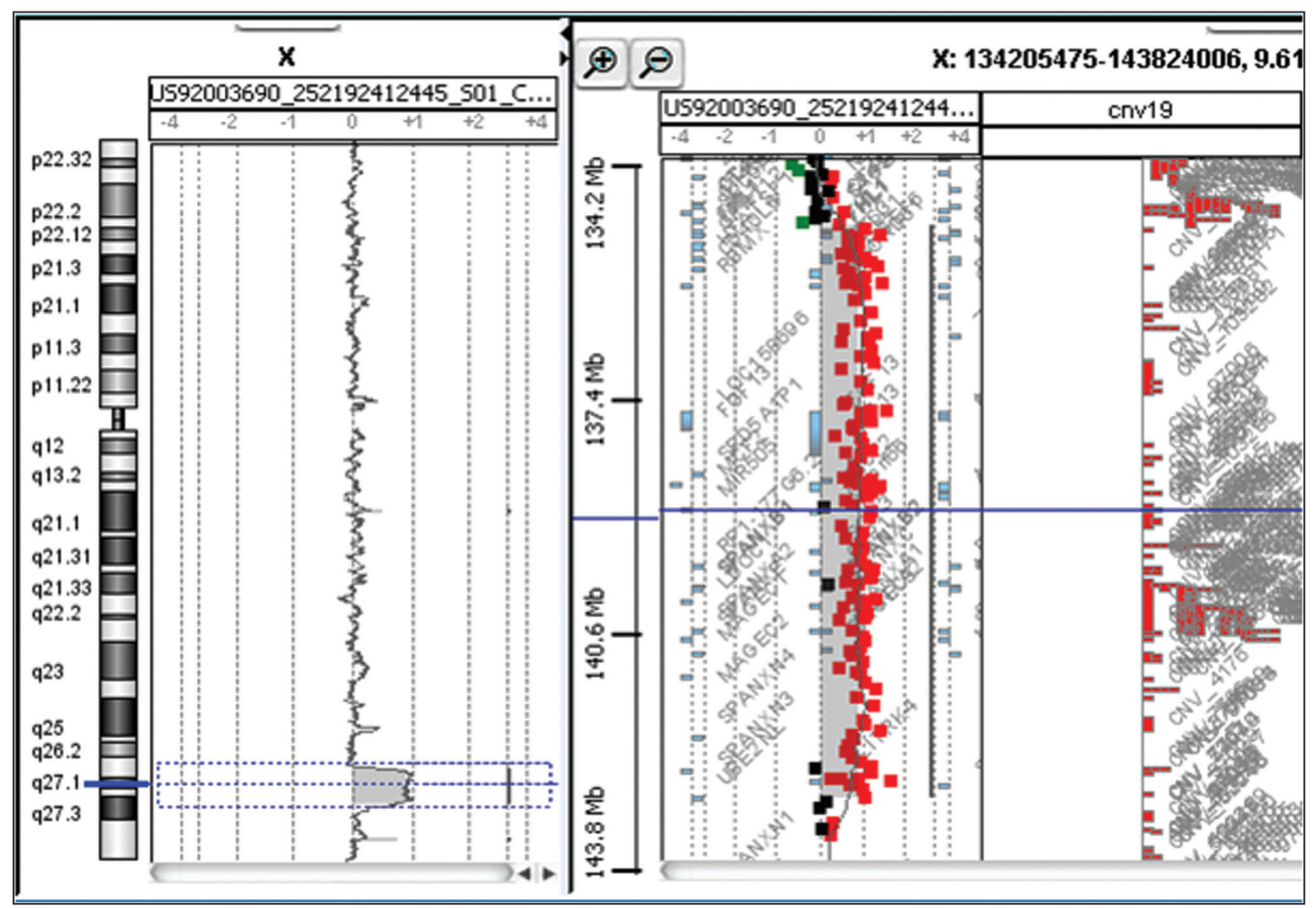

Figure 4. Array-CGH analysis showing a duplication that included Xq26.3-27.3 with the extension between 135,175,703 bp (first duplicated) and 142,971,531 bp (last duplicated). 
Table 1. Main phenotypic characteristics of patients with $S O X 3$ duplications

\begin{tabular}{|c|c|c|c|c|c|c|c|c|}
\hline Clinical findings & $\begin{array}{c}\text { Stankiewicz } \\
2005\end{array}$ & $\begin{array}{l}\text { Woods } \\
2005\end{array}$ & $\begin{array}{l}\text { Woods } \\
2005\end{array}$ & $\begin{array}{c}\text { Hol } \\
2000\end{array}$ & $\begin{array}{c}\text { Hol } \\
2000\end{array}$ & $\begin{array}{l}\text { Lagerström- } \\
\text { Fermér } 1997\end{array}$ & $\begin{array}{c}\text { Moalem } \\
2012\end{array}$ & $\begin{array}{l}\text { Our } \\
\text { case }\end{array}$ \\
\hline SOX3 abnormalities & dupXq26.2-q27.1 & $\operatorname{dupXq27.1}$ & $\operatorname{dupXq27.1}$ & dupXq26-q27 & dupXq26-q27 & dupXq25-q26 & dupXq27.1 & $\begin{array}{c}\text { dupq24.2- } \\
\text { q25.2 }\end{array}$ \\
\hline $\operatorname{Sex}(\mathrm{M}: \mathrm{F})$ & $\mathrm{F}$ & $\mathrm{M}^{1}$ & $\mathrm{M}^{1}$ & M & M & $6(\mathrm{M})$ & M & M \\
\hline Ancestry & Caucasian & Caucasian & Caucasian & Caucasian & Caucasian & NA & Asian & Caucasian \\
\hline Familial history & $t^{2}$ & NA & NA & - & - & NA & - & $t^{3}$ \\
\hline Miscarriage history & + & NA & NA & + & + & NA & - & + \\
\hline Pregnancy & uncomplicated & NA & NA & complicated ${ }^{4}$ & complicated $^{5}$ & NA & diabetes & uncomplicated \\
\hline Delivery & spontaneous & NA & NA & spontaneous & caesarean $^{6}$ & NA & spontaneous & caesarean 7 \\
\hline Age (yrs. mo) & 12.6 & 7.0 & 0.2 & 0.2 & 0.2 & NA & 0.5 & 3.11 \\
\hline Midparental height (SDS) & -1.72 & 0.20 & 0.20 & NA & NA & NA & NA & 0.50 \\
\hline Maternal height (SDS) & -1.73 & 0.70 & 0.70 & NA & NA & NA & NA & -0.10 \\
\hline Birth weight (SDS) & 0.17 & NA & NA & 0.27 & -0.53 & NA & 0.19 & 0.10 \\
\hline Birth length (SDS) & -2.50 & NA & NA & -1.20 & NA & NA & NA & 0.40 \\
\hline Neonatal symptoms & - & $t^{8}$ & $+^{9}$ & + & $\cdot$ & NA & $\cdot$ & - \\
\hline Postnatal growth failure (SDS) & -3.29 & -2.80 & -3.80 & $++^{10}$ & $\mathrm{NA}^{11}$ & $+(\mathrm{NA})$ & - & -2.40 \\
\hline Puberty & delayed & normal & & NA & & NA & & \\
\hline Dysmorphisms & + & NA & NA & + & + & NA & $\cdot$ & $\cdot$ \\
\hline Ocular abnormalities & ND & ND & ND & ND & ND & NA & ND & $++^{12}$ \\
\hline Cryptorchidism & & $\cdot$ & + & NA & - & NA & $\cdot$ & - \\
\hline Genital malformations & & NA & + & NA & - & NA & $+t^{13}$ & - \\
\hline Hypotonia & + & - & - & + & NA & NA & $\cdot$ & + \\
\hline Seizures & - & $\cdot$ & - & + & $\cdot$ & NA & $\cdot$ & + \\
\hline MRI/TC abnormalities & ND & + & + & - & ND & NA & ND & + \\
\hline Hypoplastic anterior pituitary & & + & + & & & NA & & + \\
\hline Infundibulum hypoplasia & & + & + & & & NA & & + \\
\hline Undescended neuropituitary & & + & + & & & NA & & + \\
\hline Corpus callosum malformed & & $++^{14}$ & - & & & NA & & + \\
\hline Developmental delay & + & - & .15 & + & + & + & $\cdot$ & + \\
\hline GH/IGF-I deficiency & $-/+^{16}$ & + & + & + & + & + & ND & + \\
\hline TSH deficiency & ND & + & + & + & - & $+(4 / 6)$ & ND & $\cdot$ \\
\hline ACTH deficiency & ND & $\cdot$ & + & + & NA & $+(1 / 6)$ & ND & $\cdot$ \\
\hline Gonadotropin deficiency & ND & NA & + & NA & NA & $+(2 / 6)$ & $\cdot$ & ND \\
\hline Prolactin deficiency & ND & - & NA & NA & NA & $+(2 / 4)$ & ND & - \\
\hline Other & $+{ }^{17}$ & & & $+t^{18}$ & $++^{19}$ & & & \\
\hline
\end{tabular}

${ }^{1}$ Brothers; ${ }^{2}$ mother and maternal aunt with the same Xq26.2-q27.1 duplication, showing short stature, dyslalia, hearing impairment, premature ageing, strabismus, nystagmus, optic disc abnormality, and reduced visual field; ${ }^{3}$ see pedigree in Figure $1 ;{ }^{4}$ intrauterine growth retardation and macrocephaly; ${ }^{5,6}$ fetal hydrocephalus; ${ }^{7}$ podalic presentation; ${ }^{8}$ hypoglycaemia and hyponatremia; ${ }^{9}$ neonatal hypoglycaemia; ${ }^{10}$ not present because treatments started by 6 weeks of life; ${ }^{11}$ reported $<5^{\text {th }}$ percentile at 2 months of life; ${ }^{12}$ Ocular dyspraxia and strabismus; ${ }^{13}$ bifid but well developed scrotum and penoscrotal hypospadias; ${ }^{14} \mathrm{cyst}$ within the splenium of the corpus callosum; ${ }^{15}$ hyperactivity; ${ }^{16} \mathrm{IGF}-\mathrm{I}$ level borderline low; ${ }^{17}$ hearing impairment; ${ }^{18}$ lumbar spina bifida occulta and deep sacral dimples - the skin had multiple dark lentigines; ${ }^{19}$ lumbosacral myelomeningocele, talipes equinovarus of the right foot; hydronephrosis of the right kidney. 
SOX3, FHL1 (four-and-a-half lim domains 1; OMIM 300163), CD40LG (CD40 antigen ligand; OMIM 300386), ARHGEF6 (rho guanine nucleotide exchange factor 6; OMIM 300267), ZIC3 (zinc finger protein of cerebellum 3; OMIM 300267), and F9 (coagulation factor IX; OMIM 300746). The same duplication was found in the DNA of the mother.

\section{DISCUSSION}

We describe a new case of isolated GHD in a patient with the duplication Xq26.3-27.3 comprising the SOX3 gene. The growth failure was relatively severe, but a significant catch-up growth achieved after a long-term follow-up with rhGH treatment confirmed the diagnosis of GHD.

Additional pituitary deficiencies were not recorded, and an evaluation of a possible LH/FSH deficiency will be performed in the future.

GHD has been reported in most cases of $\mathrm{SOX} 3$ involvement (Tables 1 and 2). ${ }^{10,13,14,17-19}$ Recently, Takagi described a male patient with Kabuki syndrome due to a mutation in KMT2D (Lysine-Specific Methyltransferase 2D). KMT2D is involved in the majority of cases of Kabuki syndrome, a condition that is sometimes associated with GHD. As the patient also had CPHD, the authors analysed all of the coding exons and flanking introns of currently known genes responsible for CPHD by PCR-based sequencing, discovering a mutation in $\mathrm{SOX} 3$ consisting of a deletion in the polyalanine (PA) tracts of $S O X 3$. This study provides additional evidence that $S O X 3$ mutations must be looked for in hypopituitarism.

As documented by Woods et al, the over- and under-expression of $\mathrm{SOX} 3$ is associated with significant interfamilial phenotypic variability, which may be seen in many patients even with identically sized expansions. ${ }^{10}$ To the best of our knowledge, this case is the second described isolated case of GHD after the patient who was reported by Burkitt Wright. ${ }^{18}$ In fact, GHD is more frequently associated with TSH deficiencies, the exceptions being the cases published by Hol, ${ }^{14}$ Burkitt Wright,${ }^{18}$ and ourselves; ACTH ${ }^{10,13,14,19}$ or gonadotropin ${ }^{10,13,17,18}$ deficiencies have been more rarely diagnosed, even though in many cases specific diagnostic tests were not carried out. ${ }^{16}$
Therefore, based on the evaluation of the various pituitary defects and molecular diagnoses (sequence variant and whole gene deletion or duplication), patients with duplication of $S O X 3$ could present an IGHD without the involvement of additional adenohypophyseal hormones, ${ }^{10,14,15}$ more frequently with respect to patients with $S O X 3$ sequence variants. ${ }^{10,17,19}$ Nevertheless, the cases that were reported by Hol et $\mathrm{al}^{14}$ and Woods et $\mathrm{al}{ }^{10}$ with the duplication Xq26.3-27.3 also displayed in CPHD. Other subjects with $\mathrm{SOX3}$ duplication that were described by Salomon et $\mathrm{al}^{20}$ have not been confirmed. ${ }^{21}$

Anterior pituitary hypoplasia, an absent stalk, and ectopic neurohypophysis are other useful findings that can support the diagnosis of CPHD or IGHD due to $S O X 3$ sequence variants or whole gene deletions/ duplications. ${ }^{22}$ However, some patients lack descriptions of their hypothalamic-pituitary anatomy, ${ }^{10,12-15}$ whereas others disclosed only partially the MRI characteristics that have been described as typical of SOX3 involvement. For example, in a case that was described by Woods et al, ${ }^{10}$ MRI abnormalities were absent, such as in the patient who was reported by Helle et $\mathrm{al}^{23}$ and one of the patients who was reported by Hol et al. ${ }^{14}$ Nevertheless, the case of Helle et al ${ }^{23}$ showed hyperphagia, most likely with a hypothalamic origin without other typical SOX3 involvement characteristics; this patient did not have hypopituitarism.

Available evidence demonstrates that either the over- or under-expression of $S O X 3$ can result in the perturbation of pituitary and hypothalamic development. ${ }^{24}$ Altered $S O X 3$ d osage may also be the causative mechanism for the X-linked hypopituitarism that is associated with infundibular hypoplasia, an ectopic/ undescended posterior pituitary, and abnormalities of the corpus callosum (with or without ID). ${ }^{10,12,17,19}$ Therefore, the presence of IGHD or CPHD in males, in particular if presenting ID, may be a useful indicator of potential defects in SOX3.

ID is frequently reported in these patients. The degree of mental retardation and the characteristics vary among patients. ${ }^{12-15,23}$ Even though mental retardation is reported in the majority of patients with SOX3 duplications, ${ }^{13-15}$ this trait was not present in the cases that were documented by Woods et $\mathrm{al}^{10}$, which were characterised by a smaller duplication 
Table 2. Main phenotypic characteristics of patients with $S O X 3$ involvement (not duplication)

\begin{tabular}{|c|c|c|c|c|c|c|c|c|}
\hline Clinical findings & $\begin{array}{c}\text { Woods } \\
2005\end{array}$ & $\begin{array}{l}\text { Woods } \\
2005\end{array}$ & $\begin{array}{c}\text { Woods } \\
2005\end{array}$ & $\begin{array}{c}\text { Alatzoglou } \\
2011\end{array}$ & $\begin{array}{c}\text { Takagi } \\
2013\end{array}$ & $\begin{array}{l}\text { Helle } \\
2013\end{array}$ & $\begin{array}{c}\text { Burkitt Wright } \\
2009\end{array}$ & $\begin{array}{c}\text { Laumonnier } \\
2002\end{array}$ \\
\hline SOX3 abnormalities & mutation $^{1}$ & mutation $^{1}$ & mutation $^{1}$ & mutation $^{2}$ & mutation $^{3}$ & delXq27.1q27.2 & mutation $^{4}$ & invXp21.3q27.1 \\
\hline $\operatorname{Sex}(M: F)$ & M & M & M & $\mathrm{F}$ & M & M & M & $\mathrm{F}$ \\
\hline Ancestry & $\mathrm{Arab}^{5}$ & $\mathrm{Arab}^{5}$ & $\mathrm{Arab}^{5}$ & NA & Asian & Caucasian & NA & NA \\
\hline Familial history & $t^{6}$ & $t^{6}$ & $+^{6}$ & NA & - & - & NA & - \\
\hline Miscarriage history & NA & NA & NA & NA & - & - & NA & . \\
\hline Pregnancy & NA & NA & NA & NA & uncomplicated & uncomplicated & NA & uncomplicated \\
\hline Delivery & NA & NA & NA & NA & spontaneous & NA & NA & spontaneous \\
\hline Age (yrs.mo) & 3.0 & 4.5 & 2.7 & 7.5 & 2.0 & 5.5 & NA & 10.9 \\
\hline Midparental height (SDS) & NA & NA & NA & NA & NA & NA & NA & NA \\
\hline Maternal height (SDS) & $\mathrm{NA}^{7}$ & $\mathrm{NA}^{7}$ & $\mathrm{NA}^{7}$ & NA & NA & NA & NA & NA \\
\hline Birth weight (SDS) & NA & NA & NA & NA & -0.70 & 2.50 & NA & 0.05 \\
\hline Birth length (SDS) & NA & NA & NA & NA & -1.90 & 2.00 & NA & -0.66 \\
\hline Neonatal symptoms & - & - & - & NA & $t^{8}$ & - & & - \\
\hline Postnatal growth failure (SDS) & -2.50 & -2.50 & -1.30 & -3.10 & -5.10 & -0.54 & NA & NA \\
\hline Puberty & delayed & delayed & NA & delayed & & & delayed & \\
\hline Dysmorphisms & . & - & - & $t^{9}$ & + & + & - & - \\
\hline Ocular abnormalities & ND & ND & ND & - & ND & ND & ND & $+^{10}$ \\
\hline Cryptorchidism & - & + & + & - & - & NA & ND & \\
\hline Genital malformations & -11 & $t^{12}$ & +12 & NA & - & NA & ND & \\
\hline Hypotonia & - & - & - & - & + & - & - & + \\
\hline Seizures & - & - & - & + & - & - & - & - \\
\hline MRI/TC abnormalities & - & + & + & $+t^{13}$ & + & - & + & ND \\
\hline Hypoplastic anterior pituitary & & + & + & . & + & $-/+$ & - & \\
\hline Infundibulum hypoplasia & & + & + & - & + & - & . & \\
\hline Undescended neuropituitary & & + & + & - & + & - & + & \\
\hline Corpus callosum malformed & & - & - & - & + & - & - & \\
\hline Developmental delay & - & - & - & - & + & + & - & + \\
\hline GH/IGF-I deficiency & + & + & + & + & + & . & + & ND \\
\hline TSH deficiency & + & + & + & + & + & . & - & ND \\
\hline ACTH deficiency & + & + & + & - & + & . & - & ND \\
\hline Gonadotropin deficiency & + & + & + & + & NA & . & + & ND \\
\hline Prolactin deficiency & NA & - & NA & - & - & $\cdot$ & NA & ND \\
\hline Other & & & & & $+t^{14}$ & $t^{15}$ & & \\
\hline
\end{tabular}

${ }^{1}$ Seven alanine residues were inserted in the normal polyalanine tract from amino acids $720-721 ;{ }^{2}$ loss of six alanine residues between codons 243 and 248 (p.A243_A248del6 or del6PA); 3 loss of seven alanine residues between codons 239 and 245 (p.Ala239_245 del7A); ${ }^{4}$ seven alanine residues insertion in the normal polyalanine tract from amino acids 234-249; ${ }^{5}$ Qatari first-degree consanguineous parents; ${ }^{6}$ mother heterozygous; ${ }^{7}$ reported as normal; ${ }^{8}$ neonatal hypoglycaemia; ${ }^{9}$ Turner-like habitus; ${ }^{10}$ strabismus; ${ }^{11}$ microrchidism; ${ }^{12}$ criptorchidism; ${ }^{13}$ enlarged anterohyphophysis; ${ }^{14}$ ventricular septal defect, atrial septal defect, mitral stenosis, and hearing loss; ${ }^{15}$ obesity and hyperphagia. 
(685.6 kb in length). Although one of these patients exhibited hyperactivity, the absence of ID in some patients ${ }^{10,17,18}$ may result from different dosage effects.

Vertebrate embryonic stem cells express the Sox 2 transcription factor, which, together with the closely related Sox1 and Sox3 proteins, forms the SoxB1 subgroup of the Sox protein family. First, Bergsland et al found that the genome-wide binding patterns of Sox 2 and Sox3 in neural precursor cells (NPCs) overlap extensively, with $96 \%$ of the Sox2-bound sites also bound by Sox $3 .{ }^{25}$ Therefore, a substantial number of the identified binding sites are part of brain-specific regulatory regions. Both high and low levels of Sox 3 can deleteriously affect normal brain function and physiology. In fact, constitutively active Sox3 leads to increased apoptosis. ${ }^{26}$

In conclusion, IGHD and CPHD are frequently reported characteristics in patients with $S O X 3$ involvement. The association with mental retardation is also typically present, more frequently in SOX3 duplication than in mutations. In the case of males with mental retardation and postnatal growth failure due to IGHD or associated with CHPD, the involvement of SOX3 may be considered.

\section{REFERENCES}

1. Cohen LE, Radovick S, 2002 Molecular basis of combined pituitary hormone deficiencies. Endocr Rev 23: 431-442.

2. Dateki S, Kosaka K, Hasegawa K, et al, 2010 Heterozygous orthodenticle homeobox 2 mutations are associated with variable pituitary phenotype. J Clin Endocrinol Metab 95: 756-764.

3. Kelberman D, Rizzoti K, Lovell-Badge R, Robinson IC, Dattani MT, 2009 Genetic regulation of pituitary gland development in human and mouse. Endocr Rev 30: 790-829.

4. Kelberman D, Dattani MT, 2007 Hypothalamic and pituitary development: novel insights into the aetiology. Eur J Endocrinol 157: Suppl 1: 3-14.

5. Dattani MT, 2005 Growth hormone deficiency and combined pituitary hormone deficiency: does the genotype matter? Clin Endocrinol (Oxf) 63: 121-130.

6. Collignon J, Sockanathan S, Hacker A, et al, 1996 A comparison of the properties of Sox-3 with Sry and two related genes, Sox-1 and Sox-2. Development 122: 509-520.

7. Kamachi Y, Uchikawa M, Kondoh H, 2000 Pairing SOX off: with partners in the regulation of embryonic development. Trends Genet 16: 182-187.
8. Pevny LH, Lovell-Badge R, 1997 Sox genes find their feet. Curr Opin Genet Dev 7: 338-344.

9. Lefebvre V, Dumitriu B, Penzo-Méndez A, Han Y, Pallavi B, 2007 Control of cell fate and differentiation by Sry-related high-mobility-group box (Sox) transcription factors. Int J Biochem Cell Biol 39: 2195-2214.

10. Woods KS, Cundall M, Turton J, et al, 2005 Over- and underdosage of SOX3 is associated with infundibular hypoplasia and hypopituitarism. Am J Hum Genet 76: 833-849.

11. Solomon NM, Ross SA, Morgan T, et al, 2004 Array comparative genomic hybridisation analysis of boys with $\mathrm{X}$ linked hypopituitarism identifies a $3.9 \mathrm{Mb}$ duplicated critical region at Xq27 containing SOX3. J Med Genet 41: 669-678.

12. Laumonnier F, Ronce N, Hamel BC, et al, 2002 Transcription factor SOX3 is involved in X-linked mental retardation with growth hormone deficiency. Am J Hum Genet 71: 1450-1455.

13. Lagerström-Fermér M, Sundvall M, Johnsen E, et al, 1997 X-linked recessive panhypopituitarism associated with a regional duplication in Xq25-q26. Am J Hum Genet 60: 910-916.

14. Hol FA, Schepens MT, van Beersum SE, et al, 2000 Identification and characterization of an Xq26-q27 duplication in a family with spina bifida and panhypopituitarism suggests the involvement of two distinct genes. Genomics 69: 174-181.

15. Stankiewicz P, Thiele H, Schlicker M, et al, 2005 Duplication of Xq26.2-q27.1, including SOX3, in a mother and daughter with short stature and dyslalia. Am J Med Genet A 138: 11-17.

16. Moalem S, Babul-Hirji R, Stavropolous DJ, et al, 2012 $\mathrm{XX}$ male sex reversal with genital abnormalities associated with a de novo SOX3 gene duplication. Am J Med Genet A 158A: 1759-1764.

17. Alatzoglou KS, Kelberman D, Dattani MT, 2009 The role of SOX proteins in normal pituitary development. J Endocrinol 200: 245-258.

18. Burkitt Wright EM, Perveen R, Clayton PE, et al, 2009 $\mathrm{X}$-linked isolated growth hormone deficiency: expanding the phenotypic spectrum of SOX3 polyalanine tract expansions. Clin Dysmorphol 18: 218-221.

19. Takagi M, Ishii T, Torii C, Kosaki K, Hasegawa T, 2013 A novel mutation in SOX3 polyalanine tract: a case of kabuki syndrome with combined pituitary hormone deficiency harboring double mutations in MLL2 and SOX3. Pituitary Dec 18.

20. Solomon NM, Nouri S, Warne GL, Lagerström-Fermér M, Forrest SM, Thomas PQ, 2002 Increased gene dosage at Xq26-q27 is associated with X-linked hypopituitarism. Genomics 79: 553-559.

21. Solomon NM, Ross SA, Forrest SM, et al, 2007 Array comparative genomic hybridisation analysis of boys with $\mathrm{X}$-linked hypopituitarism identifies a 3.9 Mb duplicated critical region at Xq27 containing SOX3. J Med Genet 
44: e75.

22. Tillmann V, Tang VW, Price DA, Hughes DG, Wright NB, Clayton PE, 2000 Magnetic resonance imaging of the hypothalamic-pituitary axis in the diagnosis of growth hormone deficiency. J Pediatr Endocrinol Metab 13: $1577-1583$.

23. Helle JR, Barøy T, Misceo D, Braaten Ø, Fannemel M, Frengen E, 2013 Hyperphagia, mild developmental delay but apparently no structural brain anomalies in a boy without SOX3 expression. Am J Med Genet A 161A: 1137-1142.
24. Hamel BC, Smits AP, Otten BJ, van den Helm B, Ropers HH, Mariman EC, 1996 Familial X-linked mental retardation and isolated growth hormone deficiency: clinical and molecular findings. Am J Med Genet 64: 35-41.

25. Bergsland M, Ramsköld D, Zaouter C, Klum S, Sandberg R, Muhr J, 2011 Sequentially acting Sox transcription factors in neural lineage development. Genes Dev 25: 2453-2464.

26. Archer TC, Jin J, Casey ES, 2011 Interaction of Sox1, Sox2, Sox 3 and Oct4 during primary neurogenesis. Dev Biol 350: 429-440. 


\title{
Diabetes mellitus in a girl with thyroid hormone resistance syndrome: a little recognized interaction between the two diseases
}

\author{
Stefano Stagi, ${ }^{1}$ Cristina Manoni, ${ }^{1}$ Valentina Cirello, ${ }^{2}$ Danila Covelli, ${ }^{2}$ Sabrina Giglio, ${ }^{3}$ \\ Francesco Chiarelli, ${ }^{4}$ Salvatore Seminara, ${ }^{1}$ Maurizio de Martino ${ }^{1}$
}

${ }^{1}$ Health Sciences Department, University of Florence, Anna Meyer Children's University Hospital, Florence; ${ }^{2}$ Department of Medical Sciences, University of Milan and Endocrine Unit, Fondazione Policlinico IRCCS, Milan; ${ }^{3}$ Genetics and Molecular Medicine Unit, Anna Meyer Children's University Hospital, Florence; ${ }^{4}$ Department of Paediatrics, University of Chieti, Chieti; Italy

\begin{abstract}
The syndrome of resistance to thyroid hormone (RTH) is characterized by elevated serum free thyroid hormones $\left(\mathrm{FT}_{4}\right.$ and $\mathrm{FT}_{3}$ ) in the presence of unsuppressed $\mathrm{TSH}$ levels, reflecting resistance to the normal negative feedback mechanisms in the hypothalamus and pituitary. The degree of resistance within peripheral tissues determines whether thyrotoxic clinical features are associated with this condition. Classic features include attention deficit hyperactivity disorder, growth delay, tachycardia, and goiter. However, other features, such as frequent ear, nose and throat infections, hearing deficit, and decreased bone mass have recently been recognized. The phenotype of RTH is variable, with most patients presenting with mild to moderate symptoms. In this report we describe a girl with familiar RTH and diabetes mellitus. This is, to our knowledge, the first report regarding this association. Nearly one year after long-term triiodothyroacetic acid (Triac) therapy, we observed a reduction of thyroid hormone levels with an amelioration of insulin resistance. The possible interactions between these disorders are discussed.
\end{abstract}

Key words: Diabetes, Genetic syndromes, Goiter, Thyroid hormone resistance

\section{INTRODUCTION}

The syndrome of resistance to thyroid hormone (RTH; MIM 188570) is characterized by elevated

\footnotetext{
Address for correspondence:

Dr. Stefano Stagi, Health Sciences Department, University of Florence, Anna Meyer Children's University Hospital, Florence, Italy, Tel.: + 39-055-5662585,

Fax: +39-055-4221012, E-mail: stefano.stagi@yahoo.it

Received 29-09-2013, Accepted 19-03-2014
}

serum free thyroid hormones (TH) in the presence of unsuppressed TSH levels, reflecting resistance to the normal negative feedback mechanisms in the hypothalamus and pituitary. ${ }^{1}$ The degree of resistance within peripheral tissues determines whether thyrotoxic clinical features are associated with this condition. ${ }^{1}$

RTH is caused by a mutation in the TH receptor (TR)- $\beta$ gene (THRB; MIM 190160). To date, more 
than 300 families with the RTH phenotype have been found to harbor mutations in this gene. ${ }^{2}$ The mutant TR interferes with the activity of normal TR to induce the clinical syndrome. ${ }^{3}$ Identification of an autosomal dominant mode of inheritance, in conjunction with the recognition that receptor mutants are functionally impaired, has led to the proposal that these abnormal proteins are able to inhibit the function of their wildtype counterparts in a dominant negative manner. ${ }^{1}$ However, sporadic de novo cases are also common, although recessive inheritance is rare. ${ }^{4}$

The phenotype of RTH is variable, with most patients presenting with mild to moderate symptoms. ${ }^{5}$ Since its first description by Refetoff et al in 1967, ${ }^{6}$ classic features have been progressively identified, including attention deficit and hyperactivity disorder, growth delay, tachycardia, and goiter. However, other features, such as frequent ear, nose and throat infections, hearing deficit, and decreased bone mass, have recently been recognized. ${ }^{5}$ As RTH patients present with elevated TH levels and goiter, frequently accompanied by some manifestations of thyrotoxicosis, the condition is often misdiagnosed as Graves' disease. However, exophthalmos has never been identified as a feature of RTH and thyroid auto-antibodies have been detected only in approximately $4 \%$ of patients. ${ }^{7}$

In these patients, an impairment of the glucose metabolism has not, to our knowledge, been reported. In this case report, we describe a girl with THR and diabetes mellitus, while we also discuss the potential mechanisms of this association.

\section{CLINICAL REPORT}

The patient, a female aged 10 years and 7 months, was referred to our Paediatric Endocrinology Unit for low body mass index (BMI) and suspected diagnosis of hyperthyroidism.

The proposita was the third-born child of a nonconsanguineous marriage delivered normally at 40 weeks after an uncomplicated pregnancy. Family history was suggestive of thyroid pathologies and type 2 diabetes mellitus (T2DM); the mother showed a history positive for goiter, hyperthyroidism, and fasting hyperglycaemia, diagnosed until adolescence. Three aunts have a history of thyroid diseases with goiter and unspecified dysthyroidism. One of these also showed impaired glucose tolerance (IGT), starting from the age of 34 years. One brother died of sudden infant death syndrome at three months of age. The other brother, 18 yrs old, was without any medical problems.

At birth, the girl's Apgar score was $10^{\mathrm{I}}-10^{\mathrm{V}}$. Birth weight was $2.6 \mathrm{Kg}$ (-1.90 SDS), length was $48 \mathrm{~cm}$ (-1.40 SDS) and head circumference $33.5 \mathrm{~cm}$ (-1.03 SDS). The neonatal congenital hypothyroidism screening was negative.

At 10 years and 7 months of age the girl weighed $26.6 \mathrm{~kg}(-1.75 \mathrm{SDS})$ and her height was $144 \mathrm{~cm}(-0.20$ SDS). BMI was 12.80 (-3.00 SDS), according Cacciari et al. ${ }^{8}$ The girl had no dysmorphic features. Her pubertal staging was B2-PH1-AH1. Her history was positive for frequent headache episodes and learning difficulty in school. At physical examination she appeared as hyperactive. There was no exophthalmos, muscle weakness or tremor. There was no erythema or onycholysis. The neurological and audiometric examinations were normal. Her blood pressure was $110 / 70 \mathrm{mmHg}$ and her pulse rate was 110 beats/min. The skeletal muscles appeared hypotrophic and the adipose tissue reduced. The patient's thyroid gland was slightly and symmetrically enlarged.

Basal hormonal investigation revealed $\mathrm{fT}_{4} 4.26$ $\mathrm{ng} / \mathrm{dL}$ (normal range: $0.8-1.9$ ), fT3 $8.02 \mathrm{pg} / \mathrm{mL}$ (normal range: 1.6-4.8), TSH $3.90 \mu \mathrm{IU} / \mathrm{mL}$ (normal range: 0.4-4). Antibodies against thyroperoxidase, thyroglobulin, and TSHR were undetectable. The fundus oculi, ECG, and echocardiography were normal.

Ultrasound examination of the neck showed an in-place, slightly enlarged thyroid gland. Bone age, performed according to the Greulich and Pyle method, was correspondent to chronological age (10 years vs. 10 years 7 months).

Other laboratory examinations showed normal haemoglobin levels (135 g/L; normal range: 120-150 $\mathrm{g} / \mathrm{L})$, mean corpuscular volume (MCV) $(75.7 \mathrm{fL}$; normal range: 75-90 fL), serum cortisol (18 mg/dL; 08 AM normal range $5-25 \mathrm{mg} / \mathrm{dL})$, adrenocorticotropic hormone (ACTH) (08 AM 35 pg/mL; normal range $9-52 \mathrm{pg} / \mathrm{mL}), 30$ 's prolactin (PRL) $(100 \mathrm{mIU} / \mathrm{L}$; normal range $63-426 \mathrm{mIU} / \mathrm{L})$, total calcium $(2.3$ $\mathrm{mmol} / \mathrm{L}$; normal range $2.2-2.7 \mathrm{mmol} / \mathrm{L}$ ), phosphate 
(1.12 mmol/L; normal range 1.09-1.4 mmol/L), 25-hydroxyvitamin D (30.5 ng/mL; normal value $>30 \mathrm{ng} /$ $\mathrm{mL}), 1,25$-dihydroxyvitamin $\mathrm{D}(65.5 \mathrm{pg} / \mathrm{mL}$; normal range: $19.9-67 \mathrm{pg} / \mathrm{mL})$; parathyroid hormone $(\mathrm{PTH})$ (37.6 pg/mL; normal range: $12-72 \mathrm{pg} / \mathrm{mL}$ ), bone alkaline phosphatase (82.3 U/L; normal range 40-140 $\mathrm{U} / \mathrm{L}$ ), osteocalcin (90 ng/mL; normal range 45-110 $\mathrm{ng} / \mathrm{mL})$, and urinary deoxypyridinoline $(46 \mathrm{nM} / \mathrm{mM}$ creatinine; normal range $30-60 \mathrm{nM} / \mathrm{mM}$ creatinine) levels. Her blood chemistry was AST 23 IU/L, ALT $36 \mathrm{IU} / \mathrm{L}, \gamma$-GTP $27 \mathrm{IU} / \mathrm{L}$, ferritin $10.5 \mathrm{ng} / \mathrm{mL}, \mathrm{HbA} 1 \mathrm{c}$ $5.6 \%$, triglycerides $138 \mathrm{mg} / \mathrm{dL}$, total cholesterol 235 $\mathrm{mg} / \mathrm{dL}$, HDL cholesterol $49 \mathrm{mg} / \mathrm{dL}$, LDL cholesterol $158 \mathrm{mg} / \mathrm{dL}$, insulin $26 \mu \mathrm{U} / \mathrm{mL}$, glycaemia $130 \mathrm{mg} / \mathrm{dL}$. No excretion of glucose in the urine was detectable.

However, an oral glucose tolerance test indicated diabetes mellitus with hyperinsulinism and insulin resistance: glycaemia was $131 \mathrm{mg} / \mathrm{dL}$ at 0 ', and 205 $\mathrm{mg} / \mathrm{dL}$ at $120^{\prime}$, whereas insulin was $34.6 \mu \mathrm{U} / \mathrm{mL}$ at $0^{\prime}$, and $78.8 \mu \mathrm{U} / \mathrm{mL}$ at $120^{\prime}$ (Table 1). Homeostasis Model Assessment - Insulin Resistance (HOMA-IR) index (11.19) and Matsuda index (1.29) indicated insulin resistance.

Coeliac disease screening was negative (IgA 129 $\mathrm{mg} / \mathrm{dL}$; anti-tissue transglutaminase (tTG) antibodies $0.5 \mathrm{U} / \mathrm{mL}$ ). The HLA was DQ2 DR3, 11 DRB3* DRw52.

Routine cytogenetic investigations revealed an apparently normal female karyotype $(46, X X)$. For the next diagnostic plan, the sequence analysis of the $T R \beta$ gene revealed a mutation in exon 9 and an amino acid alteration, namely, a substitution of valine for methonine at codon 313 (Met313Val) (Figure 1). This mutation has not, to our knowledge, been

Table 1. Thyroid hormone and TSH levels and metabolic evaluation before and after TRIAC administration

\begin{tabular}{lcc}
\hline & Before TRIAC (10 years 7 months) & After TRIAC (11 years 9 months) \\
\hline Height (SDS) & -0.20 & -0.11 \\
Weight (SDS) & -1.75 & -1.83 \\
BMI (SDS) & -3.00 & -2.78 \\
$\mathrm{FT}_{4}(\mathrm{ng} / \mathrm{dL})$ & 4.26 & 2.47 \\
$\mathrm{FT}_{3}(\mathrm{pg} / \mathrm{mL})$ & 8.02 & 5.23 \\
TSH $(\mu \mathrm{IU} / \mathrm{mL})$ & 3.90 & 2.60 \\
Total cholesterol (mg/dL & 235 & 176 \\
HDL cholesterol (mg/dL) & 49 & 53 \\
LDL cholesterol (mg/dL) & 158 & 101 \\
Triglycerides (mg/dL) & 138 & 108 \\
OGTT & & \\
Glycaemia (mg/dl) & & \\
T0' & 131 & 106 \\
T30' & 222 & 132 \\
T60' & 189 & 144 \\
T90' & 146 & 116 \\
T120' & 205 & 129 \\
Insulin $(\mu \mathrm{U} / \mathrm{mL})$ & & \\
T0' & 34.6 & 15.4 \\
T30' & 89.3 & 34.9 \\
T60' & 110.3 & 67.8 \\
T90' & 35.4 & 25.3 \\
T120' & 78.8 & 31.2 \\
HbA1c & 5.6 & 5.2 \\
\hline
\end{tabular}




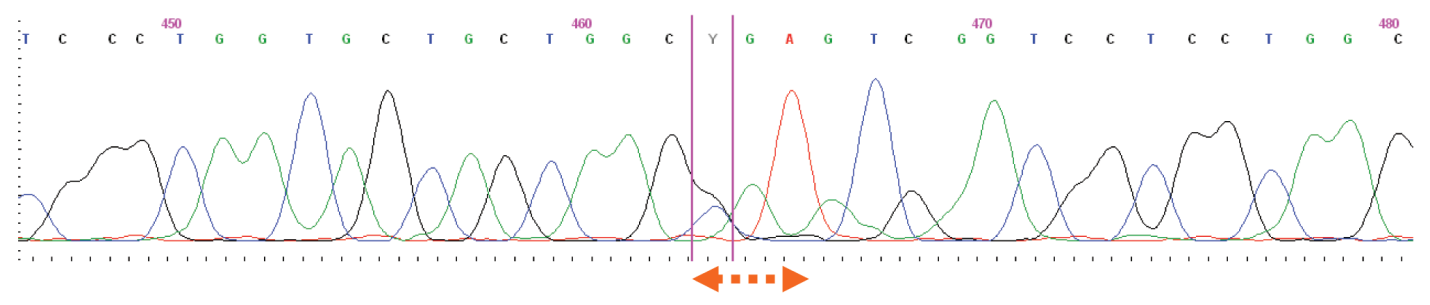

a) (PRIMER FORWARD)

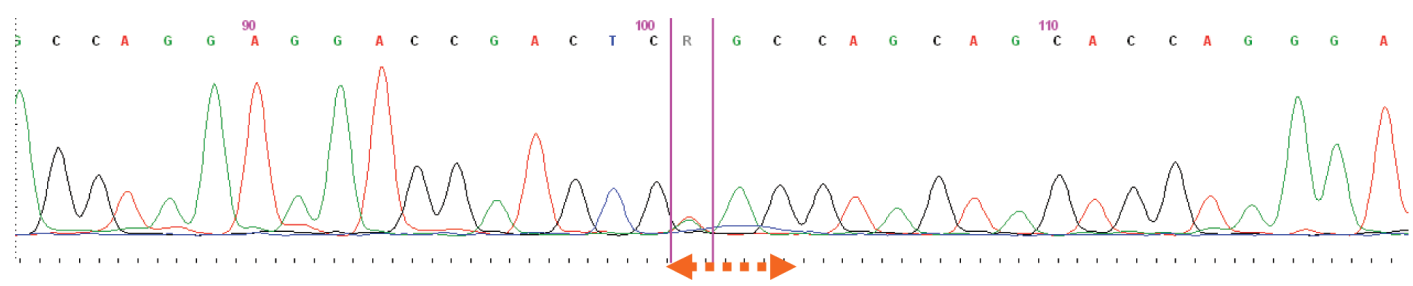

b) (PRIMER REVERSE)

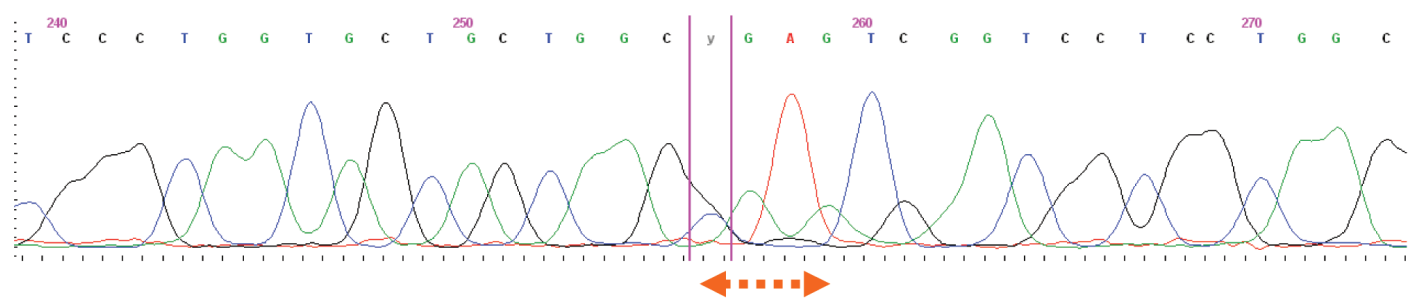

c) (PRIMER FORWARD)

Figure 1. Electropherogram of gene TR $\beta$ in the proposita (a and b) and the mother (c), showing a point mutation at codon 313 (Met313Val).

described in the literature. The same mutation was detected in the mother and in two maternal aunts (one with IGT).

Therefore, the proposita was started on a 3,5,5'-triiodothyroacetic acid (Triac) therapy at the initial dose of $1 \mathrm{mg} / \mathrm{d}$. At the same time, nutritional recommendations and interventions were also initiated for diabetes.

Almost one year after long-term Triac therapy (11 years 9 months of age), the girl weighed $30.2 \mathrm{~kg}$ (-1.83 SDS) and her height was $149.6 \mathrm{~cm}(-0.11$ SDS $)$. BMI was 13.49 (-2.78 SDS), according Cacciari et al. ${ }^{8}$ The girl had no dysmorphic features. The pubertal staging was B2-3-PH2-AH1.

We noted a reduction of thyroid hormone levels: $\mathrm{fT}_{4} 2.47 \mathrm{ng} / \mathrm{dL}, \mathrm{fT}_{3} 5.23 \mathrm{pg} / \mathrm{mL}, \mathrm{TSH} 2.60$. We also observed an amelioration of glucose and insulin metabolism: HbA1c was 5.2\%, basal glycaemia was 104 $\mathrm{mg} / \mathrm{dl}$, and basal insulin was $18.3 \mu \mathrm{U} / \mathrm{mL}$. However, triglycerides were $108 \mathrm{mg} / \mathrm{dL}$, total cholesterol 176 $\mathrm{mg} / \mathrm{dL}$, HDL cholesterol $53 \mathrm{mg} / \mathrm{dL}$, LDL cholesterol $101 \mathrm{mg} / \mathrm{dL}$. After a new oral glucose tolerance test, we hypothesized that glucose metabolism could be ameliorated by Triac treatment, possibly by improving insulin sensitivity (Table 1). In fact, basal glycaemia was $106 \mathrm{mg} / \mathrm{dL}$ (116 mg/dL at 120') and basal insulin $15.4 \mu \mathrm{U} / \mathrm{mL}\left(31.2 \mu \mathrm{U} / \mathrm{mL}\right.$ at $\left.120^{\prime}\right)$. HOMA index was 4.03 and Matsuda index 3.50.

\section{DISCUSSION}

Resistance to thyroid hormone is an uncommon disorder characterized by elevated circulating THs with nonsuppressed TSH levels, reflecting resistance within the hypothalamic-pituitary-thyroid axis but variable refractoriness to hormone action in peripheral tissues. ${ }^{9}$

Clinically, RTH can be divided into two entities: generalized (GRTH) and pituitary (PRTH) resistance. ${ }^{3}$ A molecular mechanism to explain these two 
clinical phenotypes has proven elusive and many authors have concluded that they are part of a spectrum of the same disorder..$^{10}$ However, most subjects with GRTH are either asymptomatic or have nonspecific symptoms and are deemed to be in a compensated euthyroid state. In contrast, a subset of affected individuals can exhibit some clinical features of hyperthyroidism, suggesting greater central or pituitary RTH than in peripheral tissues. ${ }^{11}$

A possible, partial, explanation may be tissuedependent TRs expression. In fact, although TR $\alpha 1$ and TR $\beta 1$ are expressed ubiquitously, TR $\alpha 1$ is expressed predominantly in the heart, bone, brain, and skeletal muscle, ${ }^{11}$ whereas TR $\beta 1$ is expressed more abundantly in liver, kidney, and thyroid. ${ }^{10}$ However, TR $\beta 2$ expression is limited to the pituitary gland, hypothalamus, retina, and inner ear. ${ }^{10}$

In the murin pancreas, $\mathrm{TR} \alpha 1$ is mainly expressed in $\alpha$-cells. ${ }^{12}$ On the other hand, $T_{3}$ rapidly induces Akt activation, with specific non-genomic action, in pancreatic $\beta$-cells via TR $\beta 1$. Thus, $T_{3}$ could be considered a survival factor protecting islet $\beta$-cells from apoptosis. ${ }^{13}$ Besides this, the predominance of an $\alpha$ receptor isoform in skeletal muscle may explain retention of sensitivity to $\mathrm{TH}$ by this target tissue, though some observations also support the notion that skeletal muscle is less refractory to $\mathrm{TH}$ action than the hypothalamic-pituitary axis in $\mathrm{RTH} .{ }^{11}$

Patients with RTH usually present with goiter and a euthyroid or mildly hypothyroid metabolic state. ${ }^{14}$ However, the clinical presentation of the disease may be hypothyroidism, with symptoms such as delayed growth, cognitive dysfunction, and hypercholesterolaemia and, concurrently, signs consistent with hyperthyroidism, including tachycardia, weight loss, attention deficit-hyperactivity disorder, and advanced bone age. ${ }^{15}$ The hypothyroid-like effects are presumably the consequence of mutant TR $\beta$ interference with, or inhibition of, normal $\mathrm{T}_{3}$ signalling pathways, whereas the signs reflective of hyperthyroidism result from the elevated $\mathrm{T}_{3}$ driving the activity of the TR $\alpha 1$ isoform. ${ }^{15}$

The secondary occurrence of T2DM with pituitary, adrenal, and/or thyroid diseases is a recurrent observation. ${ }^{16}$ Indeed, IGT and overt diabetes mellitus have frequently been associated with acromegaly, hypercortisolism, and hyperthyroidism. ${ }^{16}$
Several studies evaluating the genomic and nongenomic effects of $\mathrm{TH}$ on insulin secretion have been conducted in order to clarify the mechanism(s) behind the IGT observed in hyperthyroidism. ${ }^{17}$ For example, in rats treated with high and low doses of $\mathrm{T}_{4}$, fasting blood glucose levels were increased, but serum insulin levels were similar to those of controls. ${ }^{17}$ By contrast, in rats treated only with high doses of $\mathrm{T}_{4}$, after an oral glucose load, blood glucose levels were increased, but serum insulin levels were decreased. ${ }^{17}$ Hence, in animals, a deficient pancreatic $\beta$-cell response to glucose, rather than insulin resistance, seems to be responsible for the abnormal glucose tolerance. ${ }^{17}$

Notwithstanding, in humans, a recent study of Mitchell et al showed that RTH subjects exhibited insulin resistance as reflected by HOMA-IR. ${ }^{11}$ In fact, in a subgroup of five RTH patients undergoing an OGTT, the ISI tended to be significantly lower and HOMA significantly higher than controls, clearly indicating the presence of an insulin-resistance. ${ }^{11}$

One possible explanation for these discrepant results between animal models and humans may be that besides stimulating muscle fat oxidation and mitochondrial energy uncoupling in skeletal muscle, $\mathrm{TH}$ also promotes myocellular lipogenesis. A further possibility is that the liver RTH action might influence hepatic insulin sensitivity. ${ }^{11}$

A key phenotype associated with T2DM in humans is impaired mitochondrial oxidative metabolism in skeletal muscle, a pattern potentially contributing to increased lipid accumulation and impaired metabolic flexibility, in turn, central features of both insulin resistance and diabetes. ${ }^{18}$ In fact, the insulin-resistant state with hyperglycaemia may also be due to $\mathrm{TH}$ effects on mitochondrial content and activity, which is important for steroidogenesis as well as insulin secretion and action. ${ }^{19,20}$

However, in thyrotoxicosis, insulin levels are determined by two key factors: increased release of biologically inactive insulin precursors and increased insulin breakdown. $\beta$-cell response to a meal in patients with Graves' disease is characterized by a high proinsulin output in the hyperglycaemic state. ${ }^{21}$ The ratio of C-peptide to proinsulin is decreased, suggesting a defect in the cleaving process. ${ }^{22}$ The increase in cardiac output in hyperthyroidism leads to increased 
glomerular filtration rate and therefore increased insulin clearance. The combination of increased proinsulin secretion and increased insulin clearance results in a reduced steady state of circulating insulin in hyperthyroidism (Figure 2).

In addition to these secretion and clearance alterations, overweight hyperthyroid women lose their first-phase response to hyperglycaemia, demonstrating lower insulin peaks after intravenous glucose challenge, similar to what is observed in patients with early T2DM. ${ }^{23}$

Finally, an interesting aspect of our case report may be the possible effect, certainly also associated with changes related to the lifestyle of our patient, of Triac treatment on glucose metabolism. Although there is to date no scientific elucidation of the action of Triac on the insulinaemic metabolism, it is known that Triac has a higher affinity for TR $\beta 1 .{ }^{24}$ However, Triac inhibits leptin secretion and expression in white and brown adipocytes, whereas insulin has the opposite effect. ${ }^{25}$ In particular, leptin exerts antidiabetic actions that are independent of its regulation of body weight and food intake, ${ }^{26}$ correcting diabetes

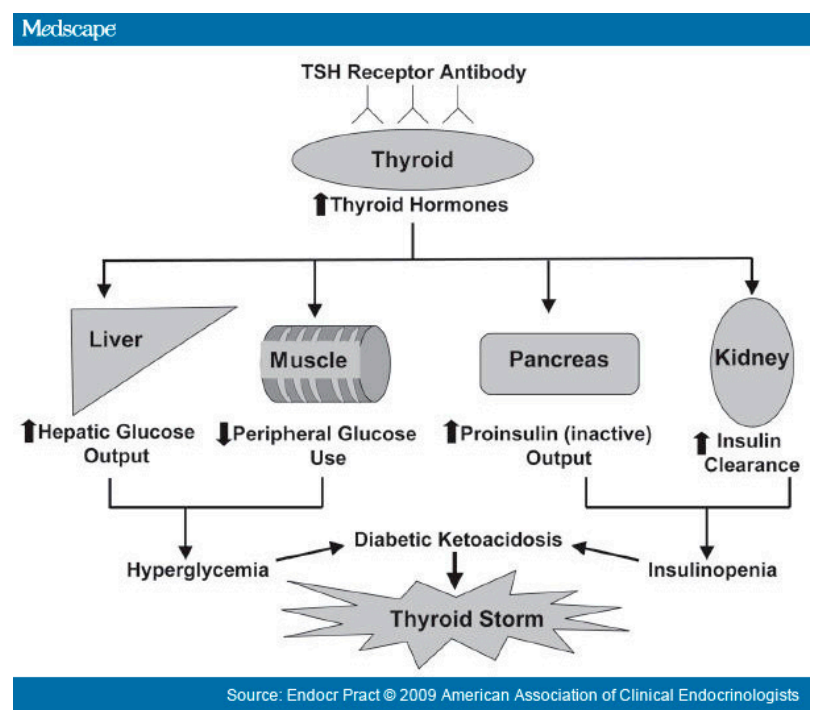

Figure 2. The effects of excess thyroid hormone on various organ systems resulting in altered glucose metabolism, potentially leading to diabetic ketoacidosis and thyroid storm. Thyroid hormone increases hepatic glucose output, decreases peripheral glucose disposal, increases inactive insulin secretion by the pancreas, and increases insulin clearance by the kidney. TSH indicates thyrotropin. in animal models of type 1 and type 2 diabetes. In addition, long-term leptin replacement therapy seems to improve glycaemic control as well as insulin sensitivity in patients with severe insulin resistance due to lipodystrophy. ${ }^{26}$

In conclusion, our data seem to show that some patients with RTH may develop an impaired glucose metabolism. We therefore suggest that glucose metabolism should be investigated in all patients with RTH.

\section{REFERENCES}

1. Gurnell M, Rajanayagam O, Agostini M, et al, 1999 Three novel mutations at serine 314 in the thyroid hormone beta receptor differentially impair ligand binding in the syndrome of resistance to thyroid hormone. Endocrinology 140: 5901-5906.

2. Refetoff S, 2005 Resistance to thyroid hormone. In: Braverman LE, Utiger RE (eds), Werner and Ingbar's the thyroid: a fundamental and clinical text. Philadelphia: Lippincott, Williams and Wilkins; pp, 1109-1129.

3. Safer JD, Langlois MF, Cohen R, et al, 1997 Isoform variable action among thyroid hormone receptor mutants provides insight into pituitary resistance to thyroid hormone. Mol Endocrinol 11: 16-26.

4. Takeda K, Sakurai A, DeGroot LJ, Refetoff S, 1992 Recessive inheritance of thyroid hormone resistance caused by complete deletion of the protein-coding region of the thyroid hormone receptor-beta gene. J Clin Endocrinol Metab 74: 49-55.

5. Refetoff S, Weiss RE, Usala SJ, 1993 The syndromes of resistance to thyroid hormone. Endocr Rev 14: 348-399.

6. Refetoff S, DeWind LT, DeGroot LJ, 1967 Familial syndrome combining deaf-mutism, stuppled epiphyses, goiter and abnormally high PBI: possible target organ refractoriness to thyroid hormone. J Clin Endocrinol Metab 27: 279-294.

7. Kourides IA, Pekonen F, Weintraub BD, 1980 Absence of thyroid-binding immunoglobulins in patients with thyrotropin-mediated hyperthyroidism. J Clin Endocrinol Metab 51: 272-274.

8. Cacciari E, Milani S, Balsamo A, et al, 2006 Italian cross-sectional growth charts for height, weight and BMI (2 to 20 yr). J Endocrinol Invest 29: 581-593.

9. Hamon P, Bovier-Lapierre M, Robert M, Peynaud D, Pugeat M, Orgiazzi J, 1988 Hyperthyroidism due to selective pituitary resistance to thyroid hormones in a 15-month-old boy: efficacy of D-thyroxine therapy. J Clin Endocrinol Metab 67: 1089-1093.

10. Beck-Peccoz P, Chatterjee VK, 1994 The variable clinical phenotype in thyroid hormone resistance syndrome. Thyroid 4: 225-232. 
11. Mitchell CS, Savage DB, Dufour S, et al, 2010 Resistance to thyroid hormone is associated with raised energy expenditure, muscle mitochondrial uncoupling, and hyperphagia. J Clin Invest 120: 1345-1354.

12. Zinke A, Schmoll D, Zachmann M, et al, 2003 Expression of thyroid hormone receptor isoform alpha1 in pancreatic islets. Exp Clin Endocrinol Diabetes 111: 198-202.

13. Verga Falzacappa C, Petrucci E, Patriarca V, et al, 2007 Thyroid hormone receptor TRbeta 1 mediates Akt activation by T3 in pancreatic beta cells. J Mol Endocrinol 38: 221-233.

14. Kopp P, Kitajima K, Jameson JL, 1996 Syndrome of resistance to thyroid hormone: insights into thyroid hormone action. Proc Soc Exp Biol Med 211: 49-61.

15. Zhang XY, Kaneshige M, Kamiya Y, Kaneshige K, McPhie P, Cheng SY, 2002 Differential expression of thyroid hormone receptor isoforms dictates the dominant negative activity of mutant Beta receptor. Mol Endocrinol 16: 2077-2092.

16. Resmini E, Minuto F, Colao A, Ferone D, 2009 Secondary diabetes associated with principal endocrinopathies: the impact of new treatment modalities. Acta Diabetol 46: 85-95.

17. Fukuchi M, Shimabukuro M, Shimajiri Y, et al, 2002 Evidence for a deficient pancreatic beta-cell response in a rat model of hyperthyroidism. Life Sci 71: 1059-1070.

18. Crunkhorn S, Patti ME, 2008 Links between thyroid hormone action, oxidative metabolism, and diabetes risk? Thyroid 18: 227-237.

19. Stark R, Roden M, 2007 ESCI Award 2006. Mitochondrial function and endocrine diseases. Eur J Clin Invest 37: 236-248.

20. Abdullah N, Al-Khalidi O, Brown KJ, Reid J, Cheetham TD, 2008 Prolonged honeymoon phase in an adolescent with diabetes and thyrotoxicosis provides support for the accelerator hypothesis. Pediatr Diabetes 9: 417-419.

21. Bech K, Damsbo P, Eldrup E, et al, 1996 beta-cell function and glucose and lipid oxidation in Graves' disease. Clin Endocrinol (Oxf) 44: 59-66.

22. Beer SF, Parr JH, Temple RC, Hales CN, 1989 The effect of thyroid disease on proinsulin and C-peptide levels. Clin Endocrinol (Oxf) 30: 379-383.

23. Ohguni S, Notsu K, Kato Y, 1995 Correlation of plasma free thyroxine levels with insulin sensitivity and metabolic clearance rate of insulin in patients with hyperthyroid Graves' disease. Intern Med 34: 339-341.

24. Takeda T, Suzuki S, Liu RT, DeGroot LJ, 1995 Triiodothyroacetic acid has unique potential for therapy of resistance to thyroid hormone. J Clin Endocrinol Metab 80: 2033-2040.

25. Medina-Gomez G, Calvo RM, Obregón MJ, 2004 T3 and Triac inhibit leptin secretion and expression in brown and white rat adipocytes. Biochim Biophys Acta 1682: 38-47.

26. Coppari R, Bjørbæk C, 2012 Leptin revisited: its mechanism of action and potential for treating diabetes. Nat Rev Drug Discov 11: 692-708. 


\title{
A novel succinate dehydrogenase type $B$ mutation in an Iranian family. Its genetic and clinical evaluation
}

\author{
Ali A. Ghazi, ${ }^{1}$ Ali Mosaddegh Khah, ${ }^{1}$ Fereshteh Kamani, ${ }^{2}$ Khandan Zare, ${ }^{3}$ \\ Alireza Sadeghipour, ${ }^{4}$ Mehdi Hedayati, ${ }^{1}$ Marjan Zarif Yeganeh, ${ }^{1}$ \\ Treena Cranston, ${ }^{5}$ Ashley Grossman ${ }^{6}$
}

${ }^{1}$ Endocrine Research center, Research Institute for Endocrine Sciences, Shahid Beheshti University of Medical Sciences;

${ }^{2}$ Department of Surgery, Taleghani General Hospital, Shahid Beheshti University of Medical Sciences; ${ }^{3}$ Department of Pathology, Taleghani General Hospital, Shahid Beheshti University of Medical Sciences; ${ }^{4}$ Department of pathology, Rasool Akram medical complex, Iran University of Medical Sciences; Tehran, Iran; ${ }^{5}$ Department of Clinical Genetics, Churchill Hospital; ${ }^{6}$ Oxford Centre for Diabetes, Endocrinology and Metabolism, Churchill Hospital, University of Oxford; Oxford, UK

\begin{abstract}
Succinate Dehydrogenase-B (SDH-B) gene mutations constitute one of the most frequent forms of hereditary paragangliomas (PGL). Genetic study is advised in all cases for the evaluation of tumour behaviour, the selection of optimal management and the surveillance of the first degree relatives. There are limited data on the genetic characteristics of patients with PGLs from Middle East countries, and to our knowledge this is the first study from Iran. We present the clinical and genetic characteristics of a 29-year old woman who presented with hypertension secondary to a para-aortic PGL. She was shown to have a novel mutation in the SDH-B gene and her family was subsequently screened. We also emphasize the problems in diagnosing and treating patients in this region.
\end{abstract}

Key words: Family, Iran, Paraganglioma, Phaeochromocytoma, SDH-B

\section{INTRODUCTION}

Phaeochromocytomas (PCC) and paragangliomas (PGL) are neuroendocrine tumours arising from chromaffin cells of the adrenal medulla or extraadrenal sympathetic or parasympathetic ganglia, respectively. The incidence of these tumours is approximately 2-8 per million population per year, with

Address for correspondence:

Prof. Ashley Grossman, OCDEM, University of Oxford, OX3

7LE, UK, E-mail: ashley.grossman@ocdem.ox.ac.uk

Received: 09-10-2013, Accepted: 06-11-2013 extra-adrenal tumours comprising some $10-20 \%$ of the cases. Mostly, the term phaeochromocytoma is used for all catecholamine-secreting tumours located either in adrenal or in extra-adrenal locations, while the term paraganglioma is used only for head-andneck tumours originating from the parasympathetic system. Several authors, however, restrict the term PCC to specifically adrenal tumours and label all others as extra-adrenal PGLs. ${ }^{1,2}$ Most PGLs are located in the abdominal or pelvic region and may present with severe hypertension due to the secretion of large amounts of adrenaline or noradrena- 
line from the tumour. PGLs develop as sporadic or hereditary forms, but germline mutations are now considered to be responsible for some $30-40 \%$ of patients presenting with phaeochromocytomas and $50 \%$ of those with paragangliomas. Mutation in the succinate dehydrogenase-B gene (SDH-B), which encodes for subunit B of the complex enzyme, has been shown to be one of the most prevalent forms of the hereditary variants of PGL, with a high rate of malignant transformation. ${ }^{3,4}$ There are only very limited reports on hereditary forms of PGLs in countries of the Middle East, and this is the first study addressing the clinical and genetic characteristics of an Iranian family harbouring a novel SDH-B mutation.

\section{Case presentation}

A 29-year old woman was referred to Taleghani General Hospital, Tehran, Iran, in 2011 because of persistent headache and palpitations for the previous 6 years which had recently deteriorated. Her past history was positive for a single episode of severe arterial hypertension (blood pressure $270 / 110 \mathrm{mmHg}$ ) that was incidentally discovered during maxillo-facial surgery 5 years previously. The surgery was cancelled and evaluation of the patient revealed high $24 \mathrm{~h}$ urinary metanephrine $(800 \mu \mathrm{g} / 24 \mathrm{~h}$, normal $<440 \mu \mathrm{g} /$ day $)$ and $24 \mathrm{~h}$ VMA $(17 \mathrm{mg} /$ day, normal $<12 \mathrm{mg} /$ day $)$ excretion, but with reported normal abdominal MRI and CT scans. The patient refused further evaluation and was discharged on combined adrenoceptor blockade with prazosin and atenolol. Five months prior to admission she was seen at another hospital for increasing headache and palpitations: an abdominal CT scan showed a para-aortic tumour. She underwent a percutaneous needle biopsy and the mass was diagnosed as a paraganglioma. She was referred to our hospital for further evaluation.

On physical examination her blood pressure was $170 / 110 \mathrm{mmHg}$ and heart rate was 110 beats per minute: the rest of the physical examination was negative. Routine laboratory evaluations were normal except for mild normocytic anaemia. Laboratory evaluation revealed high $24 \mathrm{~h}$ urine VMA $(20.3 \mathrm{mg} /$ day, normal <12) and normetanephrine $(3000 \mu \mathrm{g} /$ day, normal <440). Abdominal CT and an MRI showed a lobulated $3 \mathrm{~cm}$ round mass in the para-aortic region (Figure 1, black arrows).
After appropriate management of her hypertension with modification to her $\alpha$ - and $\beta$-adrenoceptor blockade, she underwent surgery and a solitary 4 by 3.5 by $2.5 \mathrm{~cm}$ mass located in the para-aortic region with an associated enlarged lymph node were removed. Histopathologic evaluation revealed a well-encapsulated tumour with a reddish-brown fleshy cut surface. The cells were bound with a delicate highly vascular fibrous stroma and arranged in well-defined nests or zellballen pattern. The tumoural cells had finely granular cytoplasm, round nuclei and prominent nucleoli (Figure 2A). Immunohistochemistry evaluation revealed positive staining for chromogranin $\mathrm{A}$ in the tumour cells, positive S100 in the sustentacular cells, a Ki-67 of $1 \%$ and negative staining for cytokeratin in the tumour cells (Figure 2B). The lymph node was free of tumour. The histopathologic diagnosis was of a paraganglioma.

Genetic analysis in Oxford on DNA samples prepared from the peripheral white cells revealed a heterozygous 3-nucleotide deletion, c.596-598 delACT (p.Tyr199del) in exon 6.

The patient's post-operative course was uneventful. Her blood pressure normalised after surgery and the patient was discharged from hospital after 5 days. On her last examination, one year after surgery, she had mild diastolic hypertension $(130 / 100 \mathrm{mmHg})$ on atenolol $50 \mathrm{mg}$ and hydrochlorothiazide $25 \mathrm{mg} /$ day without any signs or symptoms of catecholamine excess, and her urinary metanephrine excretion had normalised.

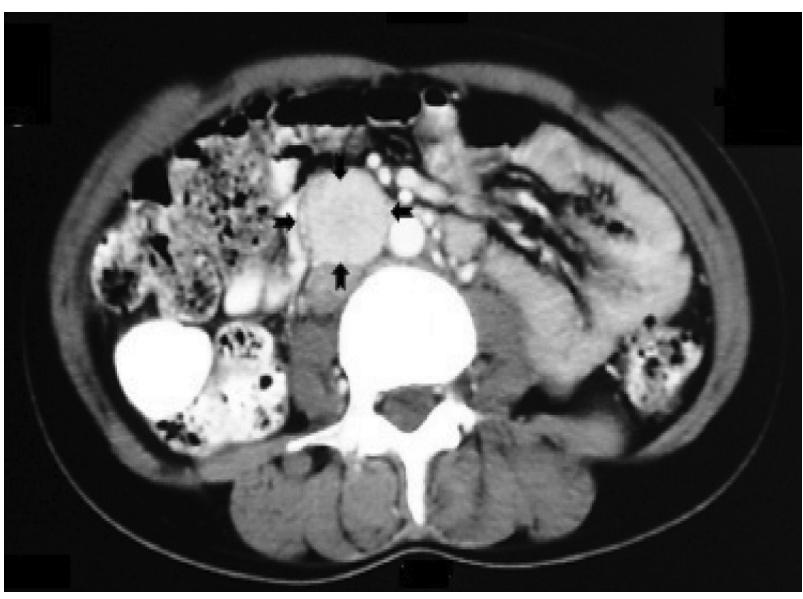

Figure 1. Abdominal CT scan showing the right sided para aortic tumour. 


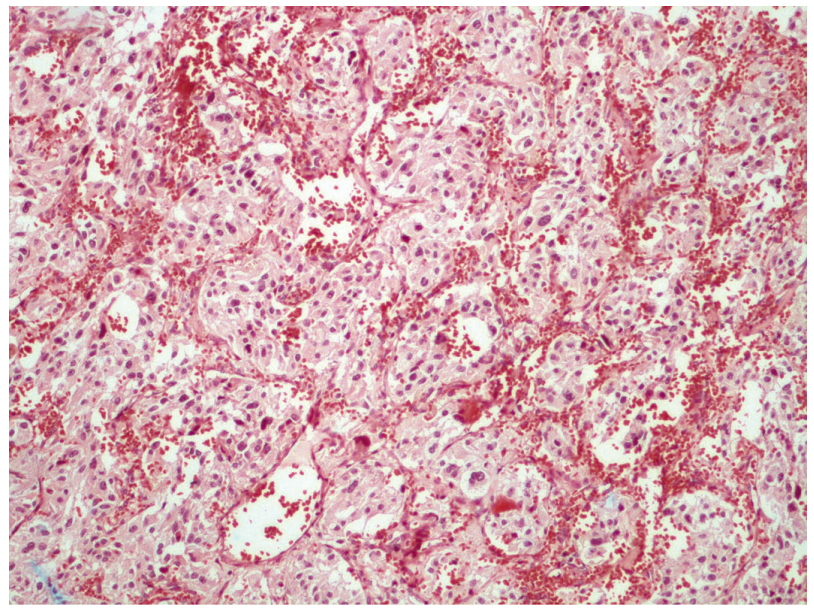

Figure 2A. Characteristic nesting pattern of tumour cells (H\&E $\mathrm{X} 100$ ).

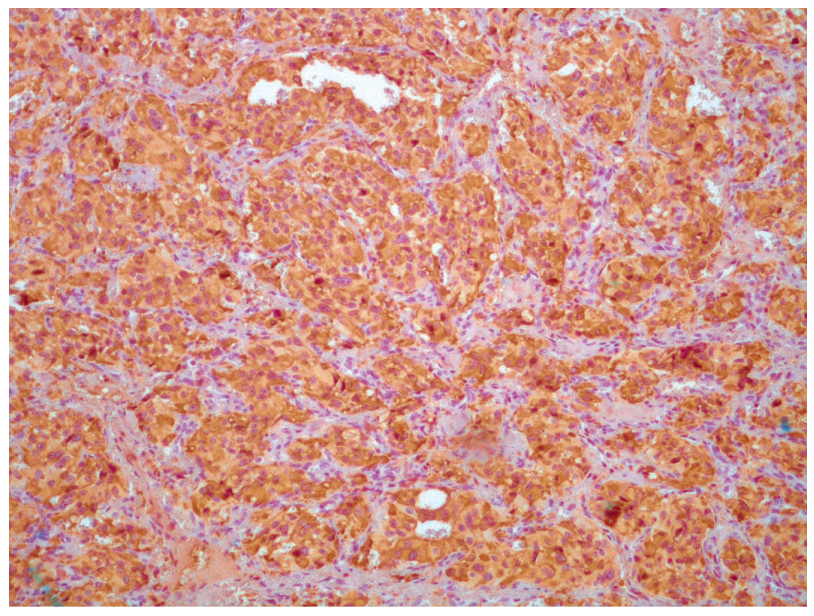

Figure 2B. Nests of tumoural cells strongly immunoreactive to chromogranin A (immunoperoxidase stain for chromogranin AX100).

The patient was the offspring of a consanguineous marriage, her parents being cousins on the maternal side. First-degree family members including her father, mother, three brothers and three sisters were also assessed. Both parents were hypertensive, 160/90 $\mathrm{mmHg}$ and $170 / 120 \mathrm{mmHg}$, but had no symptoms suggestive of catecholamine excess; no other family member agreed to clinical examination and none to biochemical assessment. However, all agreed to genetic analysis and the same mutation was found in the mother and all the siblings except one of the sisters (Figure 3).

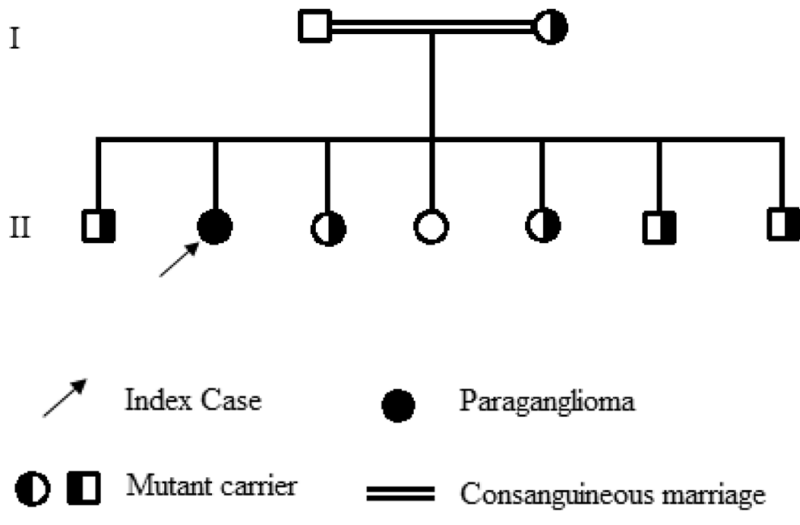

Figure 3. Pedigree of the family.

\section{DISCUSSION}

Extra-adrenal tumours comprise 10 to $20 \%$ of cases of PCC/PGLs and are more likely to be malignant in comparison to intra-adrenal tumours, $20-50 \%$ versus $10-15 \%$. Recent figures suggest that some $40-50 \%$ of patients with PGLs suffer from a hereditary form of the disease, but this will depend on the numbers of genes and also the ethnic and geographic backgrounds of the population studied. ${ }^{5-7}$ Currently, there are known to be hereditary forms of the disease that are due to mutations of principally 11 genes: $N F 1$, VHL, RET, SDH (A, B, C, D), SDHAF2, TMEM127, $H I F 2 A$ and $M A X .^{8-10}$

Succinate Dehydrogenase (SDH) is a tetrameric protein that is attached to the inner mitochondrial membrane and has 4 subunits (A, B, C and D) which are necessary for the functional activity of the protein. The enzyme catalyses succinate to fumarate in the Krebs cycle and contributes to electron transport; its main function is to link the products of the Krebs cycle to the electron transport system in order to complete glucose metabolism and ATP synthesis. The SDH-B gene is located on chromosome 1 , spans 35.4 $\mathrm{Kb}$, contains 8 exons and encodes for a 280 aminoacid protein. ${ }^{7}$

Mutations in the SDH-B gene are associated with the PGL4 syndrome. While the precise mechanism of tumorigenesis in carriers of the SDH-B mutation is unclear, it seems that increases in succinate lead to activation of hypoxia-inducible factors (HIF) due to a block of their ubiquitination: this then leads to deregulation of target genes implicated in cell prolif- 
eration, apoptosis and angiogenesis that finally results in malignant transformation. ${ }^{11-13} \mathrm{SDH}-\mathrm{B}$ gene mutation as the pathogenetic mechanism in the development of hereditary PGLs was originally described by Astuti et al in 2001; ${ }^{14}$ the following year, 2002, Neumann et al demonstrated that 66 out of $271(24 \%)$ of patients from Germany and Poland who were classified as sporadic and non-syndromic PCC/PGL indeed had hereditary forms of the disease and had a mutation in one of their corresponding genes. According to their study, SDH-B mutation positive carriers comprised $5.4 \%$ of the patients. ${ }^{3}$ Subsequently, Manelli et al showed that $32.1 \%$ of their group of 501 patients had a hereditary form of the disease. They also showed that prevalence of the hereditary form varies from $11 \%$ to $100 \%$ in different subgroups according to clinical characteristics of patients such as age, location of the tumour, the number of tumours, familial clustering or association of syndromic manifestation. SDH-B mutation carriers comprised $4.8 \%$ of their patients. ${ }^{4}$ The study conducted by a French group on 445 patients with extra-adrenal PGLs demonstrated that $54 \%$ of their patients were SDH mutation carriers, those with SDH-B mutation comprising $22 \%$ of the cases. ${ }^{15}$

More than $90 \%$ of mutations are either a point mutation or small deletions or insertions in exons 1-7. Large deletions are found in less than $10 \%$ of the cases. No mutation has yet been found in exon 8. There does not appear to be a clear genotypephenotype correlation. ${ }^{2}$

SDH-B mutation carriers may present with either thoraco-abdomino-pelvic (TAP) or head-and-neck tumours (HNPGLs). The most frequent form is a solitary, catecholamine-secreting TAP tumour. The median age at diagnosis is 34 years, but children less than 10 years may also be affected. Of SDH-B mutation carriers, $29 \%$ develop the tumour by age 30 years and $45 \%$ by age 40 years; ${ }^{16} 10 \%$ of cases are hormonally silent, which may be due an absence of tyrosine hydroxylase, the rate-limiting enzyme of catecholamine synthesis. ${ }^{17}$ According to the study by Ricketts et al on 295 patients with SDH-B mutation, the risks of TAP and HNPGL tumour development were $52 \%$ and $29 \%$, respectively, by age 60 years. ${ }^{18}$

One specific feature of SDH-B mutation carri- ers is their tendency to develop a malignant form of PGL, while they may also develop tumours of other organs such as renal cell carcinoma ${ }^{19,21}$ or gastrointestinal stromal tumours. ${ }^{20}$ Neumann et al reported that $34 \%$ of their patients with an SDH-B mutation had malignant PGLs; they also reported two cases of renal cell carcinoma and one case of papillary thyroid carcinoma among their patients. ${ }^{21}$

Genetic study is essential for risk stratification, selection of therapeutic modalities, assessment of tumour behaviour and also the evaluation of first-degree relatives. ${ }^{2,22}$ Currently, most large facilities specialising in the diagnosis and treatment of these patients will undertake multiple gene screening, although where facilities are limited the clinical characteristics of the patients such as age, family history, presence or absence of hypertension, localisation and solitary or multiple nature of the tumour can be helpful in the prioritisation of genetic screening. ${ }^{1,15}$

With regard to the current family, the mutation c.596-598 Del ACT (p.Tyr199del) is novel and has not previously been reported. As shown in Figure 3, the mother is involved, as are all of the siblings other than one sister. Evaluation of the family members such as collection of urine and imaging could not be completed because they lived in a remote area and were not willing to pursue the study, so we cannot be certain if there have been other cases with tumour among the family members, especially since two of them were hypertensive. This demonstrates the problems in screening and identifying patients with genetic disorders in societies where the economy is limited and specialist, clinicians and infrastructure are of limited availability.

The p.Tyr199 resides within a highly conserved iron-sulphur binding domain $(4 \mathrm{Fe}-4 \mathrm{~S})$, which plays a crucial role in the mitochondrial electron transport system. Residue 199 is highly conserved across species and is in a highly conserved domain of the protein. Recently, an in-frame deletion of a different aminoacid in this domain, p.Ser195del, has been reported in a patient with a PGL and in his affected son..$^{23}$ Based on these data, we believe that this mutation is highly likely to be pathogenic. With respect to the young age of the family members and low penetrance rate of SDH-B gene mutation, which is about $25-40 \%$, pro- 
longed follow-up of the patient and indeed the family is needed for accurate interpretation. ${ }^{24}$ In general, in mutation-positive subjects, regular measurement of plasma and/or urinary metanephrines, at 6-monthly or yearly intervals, is the recommended follow-up plus annual MRI of neck, thorax and abdomen and radionuclide scanning. Radilobelled ${ }^{123}$ I-MIBG is relevant therapeutically as patients can be selected for ${ }^{131}$ I-MIBG therapy, but the sensitivity is not high, especially for metastatic tumours. ${ }^{25}$ FDG-PET is preferable for sensitive detection because, as the biochemical derangements render these tumours highly inefficient in their use of glucose, they thus show a high degree of glucose uptake. Other radionuclides can be used for PET scanning but are of very limited availability. ${ }^{25}$

In conclusion, in this paper we present for the first time the clinical and genetic characteristics of a family with a novel SDH-B mutation from Iran.

\section{ACKNOWLEDGMENTS}

The authors wish to thank the family members for their cooperation in the study.

\section{FUNDING}

The study was financially supported by the Research Institute for Endocrine Sciences (RIES) Shahid Beheshti University of Medical Sciences, Tehran, Iran.

\section{REFERENCES}

1. Neumann HP, Eng C, 2009 The approach to the patient with paraganglioma. J Clin Endocrinol Metab 94: 2677-2683.

2. Fishbein L, Orlowski R, Cohen D, 2013 Pheochromocytoma/Paraganglioma: Review of perioperative management of blood pressure and update on genetic mutations associated with pheochromocytoma. J Clin Hypertens (Greenwich) 15: 428-434.

3. Neumann HP, Bausch B, McWhinney SR, et al; FreiburgWarsaw-Columbus Pheochromocytoma Study Group, 2002 Germ-line mutations in nonsyndromic pheochromocytoma. N Engl J Med 346: 1459-1466.

4. Manelli M, Castellano M, Schiavi F, et al; Italian Pheochromocytoma/Paraganglioma network, 2009 Clinically guided genetic screening in a large cohort of Italian patients with pheochromocytomas and/or functional or nonfunctional paragangliomas. J Clin Endocrinol Metab 94: 1541-1547.
5. Fishbein L, Nathanson KL, 2002 Pheochromocytoma and paraganglioma: understanding the complexities of the genetic background. Cancer Genet 205: 1-11.

6. Muth A, Abel F, Jansson S, Nilsson O, Ahlman H, Wängberg B, 2012 Prevalence of germline mutations in patients with pheochromocytoma or abdominal paraganglioma and sporadic presentation: a population-based study in Western Sweden. World J Surg 36: 1389-1394.

7. Pasini B, Stratakis CA, 2009 SDH mutations in tumorigenesis and inherited endocrine tumours: lesson from the phaeochromocytoma-paraganglioma syndromes. J Intern Med 266: 19-42.

8. Jiang S, Dahia PL, 2011 Mini review: the busy road to pheochromocytomas and paragangliomas has a new member, TMEM127. Endocrinology 152: 2133-2140.

9. Fishbein L, Merrill S, Fraker DL, et al, 2013 Inherited mutations in pheochromocytoma and paraganglioma: why all patients should be offered genetic testing. Ann Surg Oncol 20: 1444-1450.

10. Zhuang Z, Yang C, Lorenzo F, et al, 2012 Somatic HIF2A gain-of-function mutations in paraganglioma with polycythemia. N Engl J Med 367: 922-930.

11. Pollard PJ, El-Bahrawy M, Poulsom R, et al, 2006 Expression of HIF-1alpha, HIF-2alpha (EPAS1), and their target genes in paraganglioma and pheochromocytoma with VHL and SDH mutations. J Clin Endocrinol Metab 91: 4593-4598.

12. Favier J, Gimenez-Roqueplo AP, 2010 Pheochromocytomas: the (pseudo)-hypoxia hypothesis. Best Pract Res Clin Endocrinol Metab 24: 957-968.

13. López-Jiménez E, Gómez-López G, Leandro-García LJ, et al, 2010 Transcriptional profiling reveals different pseudohypoxic signatures in SDHB and VHL-related pheochromocytomas. Mol Endocrinol 24: 2382-2391.

14. Astuti D, Latif F, Dallol A, et al, 2001 Gene mutations in the succinate dehydrogenase subunit SDHB cause susceptibility to familial pheochromocytoma and to familial paraganglioma. Am J Hum Genet 69: 49-54.

15. Burnichon N, Rohmer V, Amar L, et al, 2009 The succinate dehydrogenase genetic testing in a large prospective series of patients with paragangliomas. J Clin Endocrinol Metab 94: 281-287.

16. Benn DE, Gimenez-Roqueplo AP, Reilly JR, et al, 2005 Clinical presentation and penetrance of pheochromocytoma/paraganglioma syndromes. J Clin Endocrinol Metab 91: 827-836.

17. Timmers HJ, Kozupa A, Eisenhofer G, et al, 2007 Clinical presentations, biochemical phenotypes, and genotype-phenotype correlations in patients with succinate dehydrogenase subunit B-associated pheochromocytomas and paragangliomas. J Clin Endocrinol Metab 92: 779-786.

18. Ricketts CJ, Forman JR, Rattenberry E, et al, 2010 Tumor risks and genotype-phenotype-proteotype analysis in 358 patients with germline mutations in SDHB and SDHD. Hum Mutat 31: 41-51. 
19. Vanharanta S, Buchta M, McWhinney SR, et al, 2004 Early-onset renal cell carcinoma as a novel extraparaganglial component of SDHB-associated heritable paraganglioma. Am J Hum Genet 741: 153-159.

20. Pasini B, McWhinney SR, Bei T, et al, 2008 Clinical and molecular genetics of patients with the Carney-Stratakis syndrome and germline mutations of the genes coding for the succinate dehydrogenase subunits SDHB, SDHC, and SDHD. Eur J Hum Genet 16: 79-88.

21. Neumann HP, Pawlu C, Peczkowska M, et al; 2004 European-American Paraganglioma Study Group. Distinct clinical features of paraganglioma syndromes associated with SDHB and SDHD gene mutations. JAMA 292: 943-951.

22. Erlic Z, Rybicki L, Peczkowska M, et al, 2009 Clini- cal predictors and algorithm for the genetic diagnosis of pheochromocytoma patients. Clin Cancer Res 15: 6378-6385.

23. Mhatre AN, Li Y, Feng L, Gasperin A, Lalwani AK, 2004 SDHB, SDHC, and SDHD mutation screen in sporadic and familial head and neck paragangliomas. Clin Genet 66: 461-466.

24. Jafri M, Maher ER, 2012 The genetics of phaeochromocytoma: using clinical features to guide genetic testing. Eur J Endocrinol 166: 151-158.

25. Timmers HJ, Chen CC, Carrasquillo JA, et al, 2009 Comparison of 18F-fluoro-L-DOPA, 18F-fluoro-deoxyglucose, and 18F-fluorodopamine PET and 123I-MIBG scintigraphy in the localization of pheochromocytoma and paraganglioma. J Clin Endocrinol Metab 94: 4757-4767. 


\title{
Rare case of Cushing's disease due to double ACTH-producing adenomas, one located in the pituitary gland and one into the stalk
}

\author{
Marco Mendola, ${ }^{1}$ Alessia Dolci, ${ }^{1}$ Lanfranco Piscopello, ${ }^{2}$ Giustino Tomei, ${ }^{3}$ Dario Bauer, ${ }^{4}$ \\ Sabrina Corbetta, ${ }^{1}$ Bruno Ambrosi ${ }^{1}$ \\ ${ }^{1}$ Endocrinology and Diabetology Unit, Department of Biomedical Sciences for Health, University of Milan, IRCCS \\ Policlinico San Donato, San Donato M.se, Milano; ${ }^{2}$ Endocrinology Unit, Ospedale Maggiore Trieste; ${ }^{3}$ Neurosurgical \\ Unit, Department of Biotechnology, University of Insubria, Varese; ${ }^{4}$ Pathology Unit, Department of Health Sciences, \\ University of Milan, San Paolo Hospital, Milano; Italy
}

\begin{abstract}
We describe a patient affected by Cushing's disease due to the presence of double pituitary adenomas, one located within the anterior pituitary and the other in the infundibulum associated with a remnant of Rakthe's pouch. Cure was achieved only after the infundibulum lesion was surgically removed. CASE REPORT: A 38-year-old female presented with unexplained weight gain, hirsutism, amenorrhea, asthenia, recurrent cutaneous micotic infections and alopecia. Hormonal studies indicated Cushing's disease and MRI showed an enlarged pituitary gland with a marked and homogeneous enhancement after injection of gadolinium and an enlarged infundibulum with a maximum diameter of $8 \mathrm{~mm}$. As a venous sampling of the inferior petrosal sinus after $10 \mu \mathrm{g}$ iv desmopressin stimulation revealed a central to peripheral ACTH ratio consistent with a pituitary $\mathrm{ACTH}$-secreting tumor, transphenoidal explorative surgery was performed and a 4-mm pituitary adenoma immunopositive for ACTH was disclosed and removed. Since postoperative hormonal evaluation showed persistent hypercortisolism, confirmed by dynamic tests, the patient again underwent surgery by transcranial access and the infundibulum mass was removed. Histology and immunochemistry were consistent with an ACTH-secreting adenoma. A few months after the second operation, cushingoid features were significantly reverted and symptoms improved. CONCLUSION: Although Cushing's patients bearing multiple adenomas have already been documented, the presence of two adenomas both immunohistochemically positive for ACTH is a very rare cause of Cushing's disease and this is the first report of a case of double ACTH-producing adenomas, one located in the pituitary gland and one attached to the stalk.
\end{abstract}

Key words: Cushing's disease, Double ACTH-producing adenomas, Magnetic resonance imaging, Pituitary stalk 


\section{INTRODUCTION}

Multiple pituitary adenomas are defined as simultaneous, morphologically or immunocytologically, distinct tumors ${ }^{1}$ and are classified as contiguous and clearly separated double tumors. These tumors are rarely detected among surgical specimens (0.004$0.01 \%$ ), the most frequent clinical manifestation in the reported patients being acromegaly. ${ }^{2}$ The diagnosis of double pituitary adenomas is, in most cases, based on histopathologic examination, since preoperative evaluation and MRI are able to identify multiple adenomas in only a few cases. These adenomas can belong to the same or to different hormone groups, making different combinations possible: ACTH-PRL secreting tumors appear to be the most common, followed by $\mathrm{GH}$-non functioning adenomas and $\mathrm{GH}$ PRL adenomas. ${ }^{2}$ Herein we describe, to the best of our knowledge, the first case in the recent literature of a middle-aged woman with Cushing's disease due to double adenomas, one located within the anterior pituitary and the other in the infundibulum.

\section{CASE PRESENTATION}

A 38-year-old woman was referred to our Endocrinology Unit as she had shown over the last three years progressive unexplained weight gain $(>25 \mathrm{Kg}$ bw) and hirsutism. Secondary amenorrhea had been present for six months. A polycystic ovary syndrome had been previously diagnosed and she was enrolled in a weight reduction program, without, however, any improvement. Moreover, one year before she developed typical hypercortisolemic clinical features including weight gain, depression, alopecia, profound asthenia, recurrent cutaneous micotic infections. She denied current use of alcohol. She did not suffer from other multiple endocrine neoplasia (MEN1) or Carney complex clinical manifestations.

On physical examination, vital signs were normal, weight was $90 \mathrm{~kg}$ and height was $169 \mathrm{~cm}$ (BMI 31.5 $\mathrm{kg} / \mathrm{m}^{2}$ ). Other clinical signs, e.g. moon face with mild plethora, truncal obesity, alopecia, thin skin with many bruises and abdominal striae, suggested the presence of Cushing's syndrome.

Her morning plasma ACTH and serum cortisol levels were $40-70 \mathrm{pg} / \mathrm{ml}$ and $497-821 \mathrm{nmol} / \mathrm{L}$, respec- tively. Both overnight low dose $(1 \mathrm{mg})$ dexamethasone (DXM) and low dose (2 mg for 2 days) DXM test showed an inadequate suppression of her serum cortisol (390 nmol/L/dl and $355 \mathrm{nmol} / \mathrm{L}$, respectively; normal suppression $<50 \mathrm{nmol} / \mathrm{L}$ ). Hypercortisolism was further confirmed by elevated urinary free cortisol (407-960 nmol/24h; normal range $35-275 \mathrm{nmol} / 24 \mathrm{~h}$ ). High dose dexamethasone suppression test $(8+8$ $\mathrm{mg}$ for 2 days) revealed suppression of both serum cortisol (from 868 to $108 \mathrm{nmol} / \mathrm{L}$ ) and UFC (urinal free cortisol) levels (from 960 to $59 \mathrm{nmol} / 24 \mathrm{~h}$ ). A corticotropin releasing hormone test (CRH; $1 \mathrm{mcg}$ / $\mathrm{kg} \mathrm{bw}$ ) revealed greatly increased levels of both cortisol and ACTH (by $+76 \%$ and $+92 \%$, respectively, over baseline).

A positive response to ACTH (from 43 to 112 $\mathrm{pg} / \mathrm{ml},+160 \%$ ) and cortisol levels (from 507 to 757 $\mathrm{nmol} / \mathrm{L},+49 \%$ ) was found also after desmopressin stimulation (DDAVP; $10 \mathrm{mcg}$ i.v.).

Magnetic resonance imaging (MRI) of the hypothalamic-pituitary region revealed an enlarged pituitary gland with a marked and homogeneous enhancement after gadolinium i.v. Indeed, the infundibulum was enlarged with a maximum diameter of $8 \mathrm{~mm}$ and impinged on the optic chiasm (Figure 1). Visual field evaluation revealed a minimal peripheral loss. As MRI did not identify a typical intrapituitary tumor, the patient underwent bilateral inferior petrosal sinus sampling during desmopressin stimulation. The central to peripheral ratio of ACTH was 6.17 on the

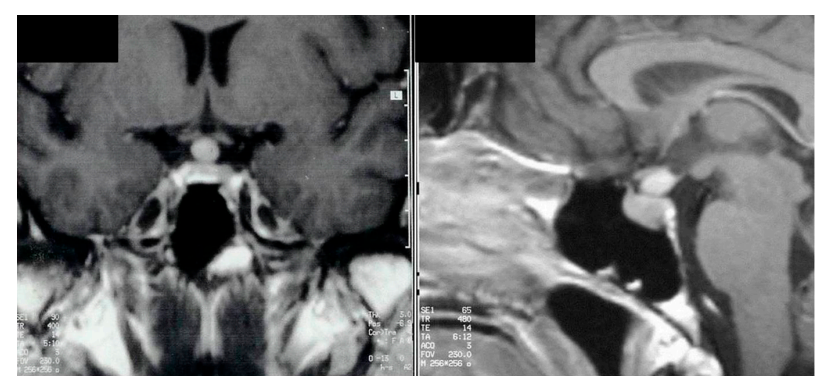

Figure 1. On the left, coronal T1-weighted, contrast-enhanced MR imaging showing a rounded mass located at the level of the pituitary stalk, over the pituitary gland, that appears intact; on the right, sagittal T1-weighted, contrast-enhanced MR imaging confirming the presence of a round mass located at the level of the pituitary stalk, over the pituitary gland, which in this plane seems enlarged in volume. 
left side after desmopressin, suggesting a possible corticotropinoma on that side. No pituitary hormones deficiencies were present.

The patient underwent transsphenoidal surgery to search for an intrapituitary microadenoma. After careful exploration of the pituitary gland, a grey soft tumor was identified and completely removed. The surgical specimen consisted of $4 \mathrm{~mm}$ of tissue and microscopically showed fragments of pituitary adenoma. The adenoma cells were immunoreactive for ACTH (Figure 2A) and immunonegative for $\mathrm{LH}$, FSH, GH, PRL and chromogranin A.

Despite these findings, postoperative hormonal evaluation showed persistent hypercortisolism.

Thereafter, based on the preoperative MRI findings, we strongly suspected that the lesion observed in the enlarged infundibulum might be a further source of ACTH secretion. Thus, one month later the patient underwent a second operation in order to remove the infundibulum lesion by transcranic access. Histological examination revealed remnants of Rakthe's pouch associated with a pituitary microadenoma. Immunohistochemical staining again demonstrated a positive reaction for ACTH (Figure 2B) and negative ones for LH, FSH, PRL, GH and chromogranin A.

Soon after the operation, ACTH and cortisol levels decreased to $4-7 \mathrm{pg} / \mathrm{ml}$ and $11-57 \mathrm{nmol} / \mathrm{L}$, respectively, (Table 1). In the subsequent days, weakness, fatigue, hypotension and skin pallor, indicative of a hypoadrenal condition, progressively ensued. As signs of central hypopituitarism $(\mathrm{LH}<0.1 \mathrm{mIU} / \mathrm{ml}$, FSH $<0.1 \mathrm{mIU} / \mathrm{ml}$, TSH $0.01 \mu \mathrm{UI} / \mathrm{ml}$ ) appeared, hormonal replacement therapy was initiated, including cortisone acetate, l-thyroxine, desmopressin and estro-progestinic therapy.

\section{DISCUSSION}

We herein describe a patient with Cushing's disease due to the presence of two ACTH-secreting adenomas, one located within the anterior pituitary and the other in the infundibulum.

Very recently, an incidence of $2.6 \%$ of double pituitary adenomas in an unselected surgical series of 117 patients undergoing surgery for pituitary adenoma was reported. ${ }^{3}$ Previously, Kontogeorgos et al ${ }^{4}$
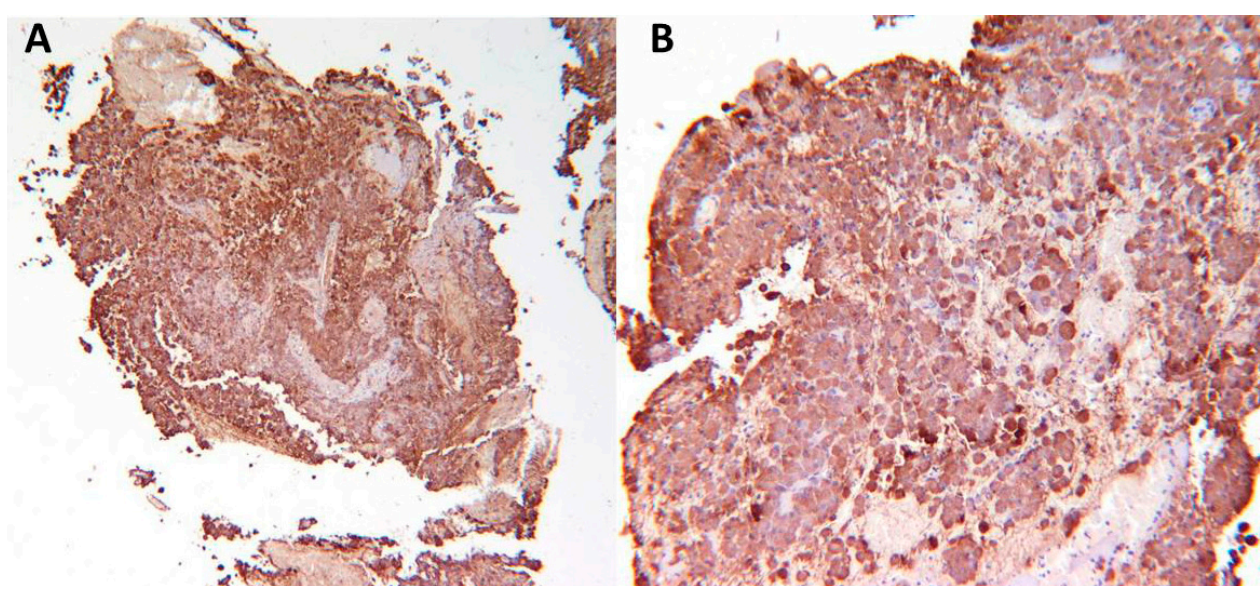

Figure 2. A: Intrapituitary microadenoma: adenomatous cells with positive immunochemistry for ACTH (4×); B: Adenoma attached to the stalk: adenomatous cells with positive immunostaining for ACTH (10×).

Table 1. Serum cortisol and plasma ACTH levels before and after surgery

\begin{tabular}{llcccc}
\hline & & Before surgery & $\begin{array}{c}\text { After 1 }^{\text {st }} \text { surgical } \\
\text { operation }\end{array}$ & $\begin{array}{c}\text { After 2 } \\
\text { operation }\end{array}$ & Normal range \\
\hline \multirow{2}{*}{ Cortisol $(\mathrm{nmol} / \mathrm{l})$} & Basal & 503 & 420 & $<28$ & $140-700$ \\
& After DXM 1mg & 390 & - & $<28$ & $<50$ \\
$\mathrm{ACTH}(\mathrm{pg} / \mathrm{ml})$ & & 43 & 71 & 7.7 & $10-90$ \\
\hline
\end{tabular}


showed that $8.9 \%$ of adenomas found at autopsy were multiple and, overall, $0.9 \%$ of autopsies detected the presence of multiple pituitary adenomas. It has also been emphasized that these tumors may occasionally co-exist with other brain tumors ${ }^{1,5-9}$ and that they may be distinguished as contiguous and clearly separated double tumors.

In autopsy specimens of double adenomas, prolactin was the most common immunoreactive secreted hormone, ${ }^{4,10}$ while most of the double adenomas in surgical series were a combination of $\mathrm{GH}$-secreting and clinically non-functioning adenomas: ${ }^{1,7,11-13}$ in fact, acromegaly has been shown to be the most frequent clinical feature in surgical series of double pituitary adenomas. ${ }^{1,5,7}$ Patients with somatotropinoma plus gonadotroph adenoma and cases of prolactinoma associated with $\mathrm{GH}$-secreting adenoma have also been described. ${ }^{1,714}$ It is of note that multiple (as many as three) intrapituitary non-functioning adenomas have additionally been documented. ${ }^{15}$

By contrast, immunostaining for adrenocorticotropic hormone in surgically resected double adenomas have rarely been described and these corticotroph adenomas were either "silent" or clinically active. In any case, isolated cases of Cushing's disease patients with double ${ }^{4,6,7,16}$ and also triple ${ }^{17}$ adenomas have been reported.

As far as double adenomas are concerned, ACTHsecreting pituitary adenomas have been described together with silent prolactinomas in two cases, ${ }^{16,17}$ with active PRL-secreting adenomas in four cases, $1,2,6,16$ with a somatotropinoma associated with acromegaly in one patient ${ }^{6}$ and with a silent $\mathrm{FSH}$-secreting adenoma in another one. ${ }^{18}$ Only one case of double pituitary adenomas ACTH-secreting has been reported recently in the literature. ${ }^{19}$

Owing to the rare occurrence of double pituitary adenomas, their pathogenesis is not known, but several possible mechanisms may be considered. One is a multicentric origin in the same pituitary, i.e. real double adenoma. The cause of multicentric adenomas might include incidental occurrence, promotion of the second adenoma growth through autocrine/paracrine pathways and a common origin. For example, studies on transgenic mice reported that several pituitary-driven growth factors can induce pituitary hyperplasia. ${ }^{20}$
Some authors suggested that one adenoma can induce the formation of another, mainly in cases of GH-secreting adenomas which release factors that may promote the proliferation of a secondary adenoma. ${ }^{1,7}$ Anyway, the role of pituitary and extrapituitary factors in inducing clonal expansion of genetically altered cells would be considered in the occurrence of multiple adenomas. In our patient, the existence of multicentric adenomas would be more plausible because the coexisting adenomas were completely separated from each other. In this context, the origin of the adenoma located within the stalk may be explained as a consequence of corticotroph basophilic invasion from the residual intermediate lobe into the posterior lobe. Starting from young adulthood, some corticotrophs in the zona intermedia proliferate into the posterior pituitary lobe and become more prominent with aging. A basophilic invasion has also been implicated as the possible origin of the extremely rare pituitary $\mathrm{ACTH}$ secreting adenomas arising from within the posterior lobe..$^{21,22}$

A poor surgical outcome has been reported in patients with double adenoma in whom the noncausative lesion was removed during the first operation. In the present case, hormonal data soon after surgery indicated the persistence of hypercortisolism and led the neurosurgeon to remove the lesion suspected at preoperative MR imaging of an enlarged infundibulum. The early occurrence of hypoadrenalism in the postoperative days indicated that a second corticotropinoma was resected: in fact, both tumors stained for ACTH on immunohistochemistry.

It is noteworthy in this regard that $\mathrm{ACTH}$-secreting adenomas originating in, or extending into, the pituitary stalk have been previously observed. ${ }^{23}$ These observations, together with the present case of double ACTH-producing adenomas, one in the anterior pituitary and the other attached to the pituitary stalk, once again emphasize that double ACTH-secreting adenomas may occur and that they need careful surgical management to recognize multiple lesions and to achieve a clinical resolution of Cushing's disease. On the other hand, the normalization of hormone hypersecretion cannot be achieved when the co-existing hypersecreting tumor is missed during surgery. In our case, the preoperative MR imaging of an enlarged infundibulum enabled us to discover, 
after the surgical failure, a second ACTH-secreting adenoma associated with a remnant of Rathke's pouch. To our knowledge, this is the first case of double ACTH-producing adenomas, one located in the anterior pituitary and the other attached to the pituitary stalk, reported in the literature.

\section{DISCLOSURE}

The authors have no financial conflict of interest.

\section{REFERENCES}

1. Kontogeorgos G, Scheithauer BW, Horvath E, et al, 1992 Double adenomas of the pituitary: a clinicopathological study of 11 tumors. Neurosurgery 31: 840-849.

2. Iacovazzo D, Bianchi A, Lugli F, et al, 2013 Double pituitary adenomas. Endocrine 43: 452-457.

3. Magri F, Villa C, Locatelli D, et al, 2010 Prevalence of double pituitary adenomas in a surgical series: clinical, histological and genetic features. J Endocrinol Invest 33: 325-331.

4. Kontogeorgos G, Kovacs K, Horvath E, Scheithauer BW, 1991 Multiple adenomas of the human pituitary. A retrospective autopsy study with clinical implication. J Neurosurg 74: 243-247.

5. Kim K, Yamada S, Usui M, Sano T, 2004 Preoperative identification of clearly separated double pituitary adenomas. Clin Endocrinol 61: 26-30.

6. Ratcliff JK, Oldfield EH, 2000 Multiple pituitary adenomas in Cushing's disease. J Neurosurg 93: 753-761

7. Sano T, Horiguchi H, Xu B, et al, 1999 Double pituitary adenomas: six surgical cases. Pituitary 1: 243-250.

8. Koutourousiou M, Kontogeorgos G, Wesseling P, Grotenhuis AJ, Seretis A, 2010 Collision sellar lesions: experience with eight cases and review of the literature. Pituitary 13: 8-17.

9. Syro LV, Horvath E, Kovacs K, 2000 Double adenoma of the pituitary: a somatotroph adenoma colliding with a gonadotroph adenoma. J Endocrinol Invest 23: 37-41.

10. Tomita T, Gates E, 1999 Pituitary adenomas and granular cells tumors. Incidence, cell type and location in 100 pituitary glands at autopsy. Am J Clin Pathol 111: 817825.

11. Tosaka M, Kohga H, Kobayashi S, et al, 2000 Double pituitary adenomas detected on preoperative magnetic resonance images. Case illustration. J Neurosurg 92: 361.

12. McKelvie PA, McNeill P, 2002 Double pituitary adenomas: a series of three patients. Pathology 34: 57-60.

13. Shimizu C, Koike T, Sawamura Y, 2004 Double pituitary adenomas with distinct histological features and immunophenotypes. J Neurol Neurosurg Psychiatry 75: 140.

14. Mohammed S, Cusimano MD, Scheithauer BW, Rotondo F, Horvath E, Kovacs K, 2010 O-Methylguanine-DNA methyltransferase immunoexpression in a double pituitary adenoma: case report. Neurosurgery 66: E421-E422.

15. Cannavò S, Curto L, Lania A, Saccomanno K, Salpietro FM, Trimarchi F, 1999 Unusual MRI finding of multiple pituitary adenomas in the gland: a case report and review of the literature. Magn Reson Imaging 17: 633-636.

16. Meij BP, Lopes MBS, Vance ML, Thorner MO, Laes ERJr, 2001 Double pituitary lesions in three patients with Cushing's disease. Pituitary 3: 159-168.

17. Pantelia E, Kontogeorgos G, Piaditis G, Rologis D, 1998 Triple adenoma in Cushing's disease: case report. Acta Neurochir 140: 190-193.

18. Oyama K, Yamada S, Hukuhara N, Hiramatsu R, Taguchi M, Imai Y, 2006 FSH-producing macroadenoma associated in a patient with Cushing's disease. Neuroendocrinol Lett 27: 733-736.

19. Andrioli M, Pecori Giraldi F, Losa M, Terreni M, Invitti C, Cavagnini F, 2010 Cushing's disease due to double pituitary ACTH-secreting adenomas: the first case report. Endocr J 57: 833-837.

20. Melmed S, 2003 Mechanism for pituitary tumorigenesis: the plastic pituitary. J Clin Invest 112: 1603-1618.

21. Hori A, Schmidt D, Kuebber S. 1999 Immunohistochemical survey of migration of human anterior pituitary cells in developmental, pathological, and clinical aspects: a review. Microscopy Research and Technique 46: $59-68$

22. Kuebber S, Ropte S, Hori A, 1990 Proliferation of adenohypophyseal cells into posterior lobe. Their normal anatomical condition and possible neoplastic potentiality. Acta Neurochir 104: 21-26.

23. Mason RB, Nieman LK, Doppman JL, Oldfield EH, 1997 Selective excision of adenomas originating in or extending into the pituitary stalk with preservation of pituitary function. J Neurosurg 87: 343-351. 


\title{
Gender identity disputed in the court of justice: a story of female to male sexual transformation in the hellenistic period, described by Diodorus Siculus
}

\author{
Anastasia K. Armeni, ${ }^{1}$ Vasiliki Vasileiou, ${ }^{2}$ George Markantes, ${ }^{1}$ Christina Damoulari, ${ }^{1}$ \\ Angelina Mandrapilia, ${ }^{1}$ Fotini A. Kosmopoulou, ${ }^{1}$ Varvara Keramisanou, ${ }^{1}$ \\ Danai Georgakopoulou, ${ }^{1}$ Neoklis A. Georgopoulos ${ }^{1}$
}

${ }^{1}$ Division of Reproductive Endocrinology, Department of Obstetrics and Gynecology, University of Patras Medical School, Patras; ${ }^{2} 1^{\text {st }}$ Department of Endocrinology, Diabetes Centre, "Alexandra" Hospital, Athens; Greece

\begin{abstract}
Cases of sexual reassignment in Greco-Roman antiquity, presenting as a pubertal female to male gender transformation, are described in the "classical" literature. Textual evidence concerning a case of androgynism, garnered by Diodorus Siculus, among other similar accounts, as an odd story of gender dispute in a court of justice, is provided in the present study. A medical interpretation of the data pertaining to this case has been attempted and is herein reported. The spontaneous virilization and post-pubertal gender inversion of the specific individual appears to have been caused by a defect either in $5 \alpha$-reductase type 2 or in $17 \beta$ hydroxysteroid dehydrogenase genes and consequent deficient enzymatic activity.
\end{abstract}

Key words: Ambiguous genitalia, Androgynism, Diodorus Siculus, Disorders of sexual differentiation, Heterosexual puberty, $5 \alpha$-reductase deficiency, $17 \beta$ HSD deficiency

\section{A. LITERAL DOCUMENTATION OF THE HERAIS} STORY: A CASE OF FEMALE TO MALE SEXUAL TRANSFORMATION DESCRIBED BY DIODORUS SICULUS

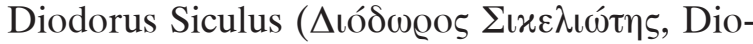
doros Sikeliotes) was a Greek historian and writer, born around 80 B.C. at the foot of the volcano of

\footnotetext{
Address for correspondence:

Neoklis A. Georgopoulos, Department of Obstetrics and Gynecology, Division of Reproductive Endocrinology, University of Patras Medical School, Rio-26500, Greece, Tel.: 2610-999835, Fax: 2610-993854, E-mail: neoklisg@hol.gr Received 31-05-13, Accepted 01-11-13
}

Etna (Aetna) in Agyrium (today Agira) in Sicily. His English translator (1933), Charles Henry Oldfather, remarks on the "striking coincidence" that one of only two known Greek inscriptions from Agyrium (I.G. $\mathrm{XIV}, 588$ ) is the tombstone of "Diodorus, the son of Apollonius". ${ }^{1} \mathrm{He}$ became famous for his monumental universal history, the "Bibliotheca Historica"1-3 (Historical Library) thought to have been written between 60 and 30 B.C. During those 30 years he was engaged in long-distance travels in order to personally collect information on different nations and tribes to be included in his history. This colossal work consisted of 40 books of which fewer than half 
have survived to the present. Fragments of the lost books are preserved in the Library of the Patriarch of Constantinople Photius (858/67-878/86 AD) and as excerpts in works by Constantine Porphyrogenitus. In this enormous epic history, Diodorus sought to incorporate all historical events from the time before the Trojan War until his own era, with special focus on the time of Alexander the Great and his successors. He decided to present his work under the name of "Bibliotheca" in recognition of the fact that he was assembling a composite work deriving from many sources. In the centuries to come, the "Bibliotheca Historica" enjoyed immense reputation and served as a reliable source for a great number of savants in the fields of philosophy, religion and history. It is therefore of particular importance that Diodorus included in his work, among events of such a huge historical significance as the Persian Wars and the decisive battles of Alexander the Great, a testimony of cases of androgynism noted in his era.

In ancient culture, humans possessing both sexes were condemned to extermination as they were regarded as miscreations and evil omens arousing terror. ${ }^{4}$ Being a rationalist, Diodorus, who lived in the age of Caesar, adopted a humanitarian view of the world, interpreting androgynous creatures as mere faults of nature. ${ }^{1}$ By including such cases in his historical work, he endeavoured to familiarize his readers with such events thus eliminating the feeling of dread at their appearance. The same attitude was also followed by other prominent historians and philosophers of his era, including Plinius the Elder, ${ }^{5}$ Phlegon ${ }^{5}$ and Titus Livius. ${ }^{6}$

Among the cases of androgynism collected by Diodorus, the story of Herais (Hoaǐs) is of especial interest, the reason for this being that, to our knowledge, this is the only description of a legal dispute over the gender of a person with ambiguous genitalia ever reported throughout ancient history.

The dating of the event is close to 145 B.C. and, in brief, the sequence of events was the following:

"In a city of Arabia, called Aves, lived a man of Macedonian origin, named Diophantus. He was married to a native woman and they had a daughter named Herais. When Herais was at the proper age, her father married her to a man named Samiades.
Samiades lived with his wife for a year and then went off on a long journey. During his absence, Herais developed a strange illness, characterized by intense inflammation at the base of her abdomen. As the swelling worsened and was also complicated by high fever, the physicians suspected that an ulceration had taken place at the mouth of the uterus. They applied remedies which would reduce the inflammation but on the seventh day, the surface of the swelling cracked and a penis and two testicles appeared in Herais' groin.

When the rupture occurred, with its sequel, neither her physician nor any other visitors were present, but only her mother and two maidservants. Although astonished by this strange phenomenon, the women provided Herais proper care and withheld the truth. Herais, having been relieved of her "illness", continued to wear feminine attire but returned to her father's house.

When Samiades returned, he asked to see his wife. Herais was ashamed and did not dare to appear in front of her husband. Thus, Samiades dragged her father to trial, claiming his wife back. The judges decided that Herais had to follow her husband. It was only then that Herais revealed the truth: mobilizing all her courage, she took off her dress and displayed her masculinity in public, screaming and asking how could some people force a man to live with another man. All present were overcome with astonishment but accepted this unusual fact. As it is said, Herais, after revealing her male nature, exchanged her woman's apparel for the garb of a young man, changed her/ his name to Diophantus and enlisted in the cavalry fighting with the king. ${ }^{2}$ As for Samiades, they say that, constrained by shame for his unnatural marriage, he made his departure from life. Thus, she who was born a woman took on a man's courage and renown, while the man proved to be less strong-minded than a woman."

\section{B. DIFFERENTIAL DIAGNOSIS OF HERAIS' CASE}

Diodorus Siculus described the story of Herais, a case of sexual reassignment, namely a female to male gender role change occurring after marriage and consisting of a female to male phenotypic transition. Herais was raised as female during childhood, was married to a man and subsequently experienced 
a sexual transformation. Given that marriages at that time were frequently agreed upon close to the time of puberty or soon after, the described female to male sexual transformation is in fact a case of heterosexual puberty. Indeed, Diodorus described the appearance of male external genitalia, including a penis-like structure and two testes, through an inflamed hypogastrial hernia. This inflammation was probably caused by a pubertal increase in the size of the testes trapped in the narrow inguinal duct. After this event Herais completely changed his/her gender assignment, rejected femininity and strongly adhered to a reassigned masculinity that led him to completely change his social orientation: he changed his name from feminine to masculine and served as a warrior in the army of the king. We can assume, therefore, that Diodorus described a case of a Disorder of Sexual Differentiation (DSD) which, despite the intra-abdominal presence of both testes, was raised as female during childhood and presented pubertal signs of virilization, leading to heterosexual puberty.

The presence of two testes in Diodorus' description of Herais' case excludes the diagnosis of virilizing adrenal or testicular tumors, gonadal dysgenesis, true hermaphroditism and female pseudohermaphroditism. ${ }^{7}$ Therefore, heterosexual puberty in this particular case will have been the result of an increase in serum androgen levels, produced by testicular tissue, leading to the development of male secondary sex characteristics.

Given that heterosexual puberty has never been reported in Androgen Insensitivity Syndrome (AIS), the most likely diagnosis in cases of female to male transition is a defect in androgen synthesis. Among all enzyme deficiencies in the pathway of testosteroneDHT synthesis are only those of $17 \beta$ hydroxysteroid dehydrogenase (HSD17B3), the enzyme converting androstenedione to testosterone, or $5 \alpha$-reductase type 2 , the enzyme converting testosterone to dihydrotestosterone (DHT). Such defects can lead to similar clinical phenotypes characterized by ambiguous genitalia appearance leading to female sex assignment at birth and heterosexual puberty. ${ }^{7}$

Differential diagnosis between $5 \alpha$-reductase type 2 and HSD17B3 deficiencies cannot be made based on clinical phenotype. $5 \alpha$-reductase type 2 converts testosterone to DHT, the major determinant of male external genitalia formation, and is highly expressed early in gestation in testicular, scrotal and phallic tissues. This undoubtedly makes its action essential for the normal development of male primary sexual characteristics in the fetus. Therefore, lack of expression of this gene leads to decreased production of DHT and development of external genitals in the neonate, simulating those of a female, as in the case of Herais. ${ }^{9}$ Depending on the molecular defect, $5 \alpha$-reductase deficiency may be manifested with a wide spectrum of clinical phenotypes, from milder ones that can be easily overlooked ${ }^{10}$ to more severe ones resembling Herais' case. ${ }^{11}$ HSD17B3 deficiency also results in genital transformation at puberty which can be quite pronounced.

$5 \alpha$-reductase type 2 deficiency promotes more considerable changes during puberty, which are attributed to the accumulated testosterone and its conversion to DHT by type 1 isoenzyme. Unlike $5 \alpha$-reductase type 2 , type 1 is not expressed in utero, therefore contributing to postnatal sexual but not to fetal differentiation. ${ }^{12}$ An age-dependent increase in the expression of the $5 \alpha$-reductase type 1 gene has been reported, rendering its action measurable during puberty and for the remainder of life in peripheral tissues, such as liver and kidney, thus compensating for $5 \alpha$-reductase type 2 deficiency.

Heterosexual puberty in cases of HSD17B3 deficiency is due to isoenzyme HSD17B5. Although HSD17B3 converts androstenedione to testosterone in the testes, HSD17B5 is responsible for extragonadal production of testosterone in peripheral tissues, such as placenta, prostate, adrenals and liver, while it slightly contributes to testosterone produced by the testes. ${ }^{13}$ Furthermore, there is an age-dependent regulatory transcription mechanism concerning HSD17B3 and HSD17B5 isoenzymes, with the former decreasing and the latter increasing with age. ${ }^{14,15}$

Nevertheless, the clinical phenotype of $5 \alpha$-reductase deficiency cannot be clearly distinguished from that of $17 \beta$ hydroxysteroid dehydrogenase deficiency, ${ }^{12}$ and even less so, needless to say, based on historical data. Therefore, in the absence of a definitive molecular diagnosis, Herais' case could be attributed to either a $5 \alpha$-reductase or a $17 \beta$ hydroxysteroid dehydrogenase 
gene deficiency, with the former being more likely.

Finally, both $5 \alpha$-reductase and $17 \beta$ hydroxysteroid dehydrogenase gene deficiencies are encountered in the region of the Eastern Mediterranean. Indeed, recent molecular analysis of cases of $5 \alpha$-reductase type 2 gene deficiency revealed a high incidence of cases in the lands around the Mediterranean Sea, many of them reported in subjects of Arabian and Greek ethnic origin. ${ }^{11}$ Similarly, prevalence of $17 \beta$ hydroxysteroid dehydrogenase gene deficiency is higher among Arabian and Mediterranean populations. ${ }^{16}$

In conclusion, based on the above contemporary appraisal of the case, we could attribute Herais' spontaneous virilization and post-pubertal gender inversion to molecular defects either in $5 \alpha$-reductase type 2 or in $17 \beta$ hydroxysteroid dehydrogenase gene expression and consequent deficient enzyme activity.

\section{REFERENCES}

1. Siculus Diodorus, Booth G (Translator), Valesius H, Rhodomannus I, Ursinus F 1814 The Historical Library of Diodorus the Sicilian in Fifteen Books to which are added the Fragments of Diodorus. London: J. Davis. Downloadable Google Books.

2. Siculus Diodorus, Oldfather CH (Translator), 1935 Library of History: Loeb Classical Library. Cambridge, MA.: Harvard University Press.

3. Siculus Diodorus, Oldfather CH (Translator), 2008 “Library”. Perseus Digital Library. Books pp, 9-17.

4. Luc Brisson 1997 Le sexe incertain: Androgynie et hermaphroditisme dans l'Antiquite gréco-romaine. Paris, Les Belles Lettres.

5. Armeni AK, Vasileiou V, Georgopoulos NA, 2014 When genotype prevails: sexual female-to-male transformation in Classical Antiquity, recorded by Gaius Plinius Secundus and Phlegon. Hormones (Athens) 13: 153-156.
6. Vasileiou V, Armeni AK, Pierris AL, Georgopoulos NA, 2012 Macrosomia and ambiguous genitalia: a long overdue answer to the citizens of Frusino. Hormones (Athens) 11:215-221.

7. Kousta E, Papathanasiou A, Skordis N, 2010 Sex determination and disorders of sex development according to the revised nomenclature and classification in 46, XX individuals. Hormones (Athens) 9: 218-131.

8. Hughes IA, Davies JD, Bunch TI, Pasterski V, Mastroyannopoulou K, MacDougall J, 2012 Androgen insensitivity syndrome. Lancet 380: 1419-1428.

9. Imperato-McGinley J, Zhu YS, 2002 Androgens and male physiology the syndrome of 5alpha-reductase-2 deficiency. Mol Cell Endocrinol 198: 51-59.

10. Skordis N, Shammas C, Efstathiou E, Sertedaki A, Neocleous V, Phylactou L, et al, 2011 Late diagnosis of 5alpha steroid-reductase deficiency due to IVS12A $>$ G mutation of the SRD5a2 gene in an adolescent girl presented with primary amenorrhea. Hormones (Athens) 10: 230-235.

11. Maimoun L, Philibert P, Cammas B, et al, 2011 Phenotypical, Biological, and Molecular Heterogeneity of 5 $\alpha$-Reductase Deficiency: An Extensive International Experience of 55 Patients. J Clin Endocrinol Metab 96: 296-307.

12. Thiele S, Hoppe U, Holterhus PM, Hiort O, 2005 Isoenzyme type 1 of 5alpha-reductase is abundantly transcribed in normal human genital skin fibroblasts and may play an important role in masculinization of 5alpha-reductase type 2 deficient males. Eur J Endocrinol 152: 875-880.

13. George MM, New MI, Ten S, Sultan C, Bhangoo A, 2010 The clinical and molecular heterogeneity of 17ßHSD-3 enzyme deficiency. Horm Res Paediatr 74: 229-240.

14. Hoppe U, Holterhus PM, Wunsch L, et al, 2006 Tissuespecific transcription profiles of sex steroid biosynthesis enzymes and the androgen receptor. J Mol Med 84: 651-659.

15. Faienza MF, Cavallo L, 2012, 17ß-Hydroxysteroid Dehydrogenase Type 3 Deficiency: Diagnosis, Phenotypic Variability and Molecular Findings. Steroids - Basic Science ISBN: 978-953-307-866-3.

16. Rosler A, 200617 beta-hydroxysteroid dehydrogenase 3 deficiency in the Mediterranean population. Pediatr Endocrinol Rev 3: Suppl 3: 455-461. 


\title{
Severe hypothyroidism due to autoimmune thyroiditis in a child: a one-year follow-up
}

\author{
Francesco Vierucci, Graziano Cesaretti
}

Pediatric Endocrinology, Department of Pediatrics, "S. Chiara” University-Hospital, Pisa, Italy

Dear Editor,

Autoimmune thyroiditis is considered to be the most common autoimmune condition, with a female preponderance of $2: 1{ }^{1,2}$ While its overall prevalence peaks in adulthood, autoimmune thyroiditis may affect children and adolescents, particularly during early to mid-puberty. ${ }^{3}$ Hypothyroidism secondary to autoimmune thyroiditis may develop insidiously with slow and progressive physical changes that may remain unrecognized even to parents. ${ }^{4}$ Nevertheless, early recognition of symptoms and signs of hypothyroidism is important to prevent its negative effects on growth, metabolic function, and intellectual performance.

An eleven-year-old Caucasian boy was evaluated for poor linear growth, with normal weight gain starting at the age of six and never investigated previously. Physical examination showed short stature (height: -2.2 SDS; target height: 0.1 SDS) with overweight (body mass index: +1.7 SDS), bradycardia, sparse-brittle hair, dry and thickened skin with myxedema involving the extremities (Figure 1). The child appeared

Key words: Autoimmune thyroiditis, Childhood, Hypothyroidism, Myxedema

Address for correspondence:

Francesco Vierucci, M.D., Pediatric Endocrinology, Department of Pediatrics, "S. Chiara" University-Hospital, via Roma 67, 56126 Pisa, Italy, Tel.: +39 050 992101, E-mail: vieruf@hotmail.it

Received 16-01-2014, Accepted 19-03-2014 severely hypokinetic with apathy and mildly reduced intellectual performance (low-normal IQ score of 81, assessed by Wechsler Intelligence Scale for Children, third edition-WISC III). He also reported fatigue, constipation, sleepiness, impaired school performance, and cold intolerance.

Bone age was severely delayed (6 years as assessed by the Greulich and Pyle standard). Serum thyroid-stimulating hormone (TSH) levels were remarkably increased $(1,648 \mathrm{mIU} / \mathrm{L})$ with undetectable concentrations of thyroid hormones and elevated anti-microsomal and anti-thyroid peroxidase antibodies titers $(>2,040 \mathrm{IU} / \mathrm{mL}$ and $>1,020 \mathrm{IU} / \mathrm{mL}$, respectively). Thyroid ultrasound disclosed hypoechoic, inhomogeneous, and atrophic thyroid gland, confirming the diagnosis of severe hypothyroidism secondary to autoimmune thyroiditis.

Biochemical evaluation also revealed hypertransaminasemia (aspartate aminotransferase 151 $\mathrm{U} / \mathrm{L}$, alanine aminotransferase $117 \mathrm{U} / \mathrm{L}$ ), dyslipidemia (total cholesterol $360 \mathrm{mg} / \mathrm{dL}$, high-density lipoprotein cholesterol $29 \mathrm{mg} / \mathrm{dL}$, low-density lipoprotein cholesterol $294 \mathrm{mg} / \mathrm{dL}$, triglycerides $221 \mathrm{mg} / \mathrm{dL}$ ), and rhabdomyolysis (creatine kinase 6,883 U/L). Cerebral MRI showed a pituitary mass of $18 \times 12 \times 10$ $\mathrm{mm}$ extending into the suprasellar region, compatible with pituitary hyperplasia.

Low-dose levothyroxine administration was promptly started and progressively increased [0.5 $\mu \mathrm{g} / \mathrm{Kg} / \mathrm{die}$ (considering ideal weight according to height) for 2 weeks followed by $0.75 \mu \mathrm{g} / \mathrm{Kg} / \mathrm{die}$ for 1 month, $1 \mu \mathrm{g} / \mathrm{Kg} /$ die for 1 month and $1.5 \mu \mathrm{g} / \mathrm{Kg} /$ die for 


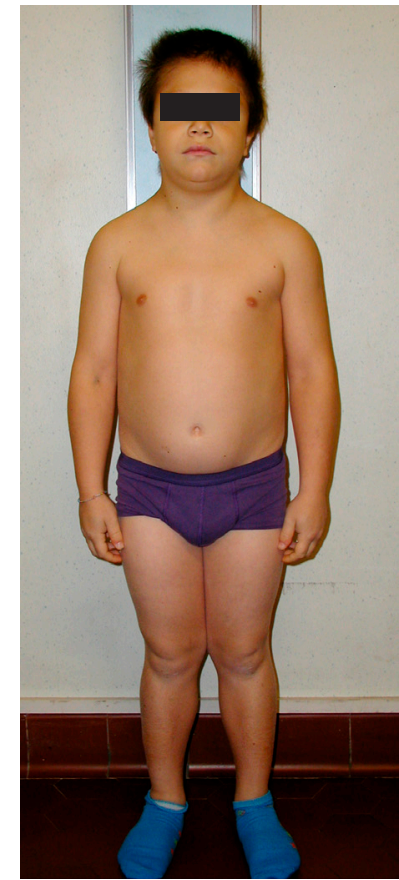

Figure 1. The patient at diagnosis with apathy, myxedema of the extremities, and sparse-brittle hair.

1 month, continuing with $2 \mu \mathrm{g} / \mathrm{Kg} /$ die afterwards]. Free thyroxine (fT4) and TSH levels normalized after 3.5 and 6 months of therapy, respectively. After twelve months of follow-up height improved to - 1.7 SDS. The child displayed normal hair, complete resolution of myxedema, normalization of biochemical alterations and intellectual performance (IQ score 92) (Figure 2). Finally, MRI showed the resolution of pituitary hyperplasia.

The case we describe herein illustrates that forms of severe juvenile hypothyroidism are still relevant today. It seems unbelievable that nobody had recognized the progressive onset of severe hypothyroidism before the child developed severe multi-organ complications. Moreover, this case demonstrates that levothyroxine administration significantly improves the changes due to long-lasting hypothyroidism acquired during childhood.

A careful monitoring of growth is essential to avoid late diagnosis of diseases with possible longterm sequelae, such as acquired hypothyroidism. A child with poor linear growth should always be adequately investigated. Finally, the detection of a child with impaired linear growth associated with normal

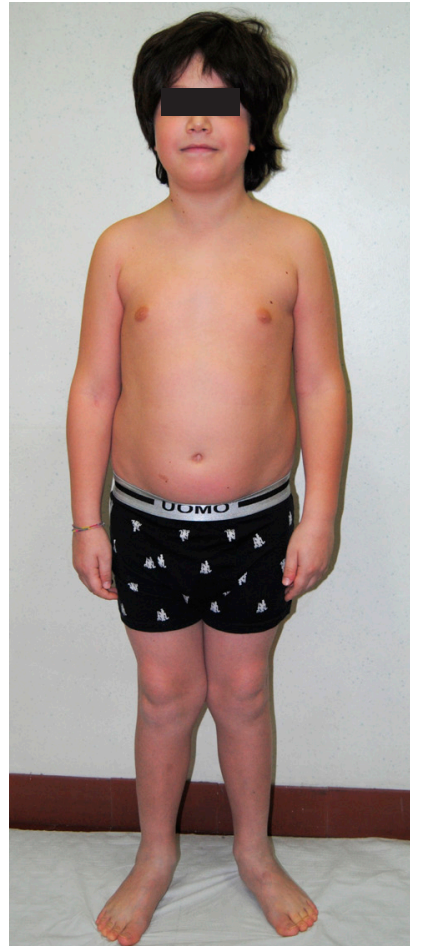

Figure 2. The patient after twelve months of therapy with levothyroxine. The resolution of myxedema and the normalization of hair growth were noticeable in association with the disappearance of apathy.

weight gain should always arouse the suspicion of hypothyroidism. ${ }^{4}$

\section{DISCLOSURE STATEMENT:}

The authors have nothing to disclose.

\section{PATIENT CONSENT}

Obtained from the parents.

\section{REFERENCES}

1. Saravanan P, Dayan CM, 2001 Thyroid autoantibodies. Endocrinol Metab Clin North Am 30: 315-337.

2. Lafranchi S, 1992 Thyroiditis and acquired hypothyroidism. Pediatr Ann 21: 29-39.

3. Cappa M, Bizzarri C, Crea F, 2011 Autoimmune thyroid diseases in children. J Thyroid Res 2011: 675703.

4. Büyükgebiz A 2007 Newborn screening, hypothyroidism in infants, children and adolescents. In: Krassas GE, Rivkees SA, Kiess W (eds), Diseases of the thyroid in childhood and adolescence. Pediatr Adolesc Med Basel, Karger; pp, 128-141. 


\title{
Genetic analysis does not confirm non-classical congenital adrenal hyperplasia in more than a third of the women followed with this diagnosis
}

\author{
Valeria Alcantara-Aragon, ${ }^{1}$ Silvia Martinez-Couselo, ${ }^{2}$ Diana Tundidor-Rengel, ${ }^{1}$ \\ Susan M. Webb, ${ }^{1,5}$ Gemma Carreras, ${ }^{3}$ Juan J. Espinos, ${ }^{4}$ Ana Chico, ${ }^{1}$ \\ Francisco Blanco-Vaca, ${ }^{2}$ Rosa Corcoy ${ }^{1,6}$
}

${ }^{1}$ Endocrinology, ${ }^{2}$ Biochemistry, ${ }^{3}$ Pediatrics, and ${ }^{4}$ Gynecology Departments, Hospital de la Santa Creu I Sant Pau, ${ }^{5}$ CIBERER 747, ${ }^{6}$ CIBERBBN; Barcelona, Spain

Dear Sir,

Non-classical congenital adrenal hyperplasia (NC$\mathrm{CAH}$ ) due to 21-hydroxylase deficiency is one of the most frequent autosomal recessive diseases, with an estimated prevalence of 1 in 1000. It may manifest at different stages in life. In late childhood, it may present with advanced skeletal maturation, accelerated growth or premature pubarche. In adult women, the disease usually presents with a hyperandrogenic syndrome including hirsutism, acne, androgenic alopecia, anovulation, menstrual dysfunction, and infertility. ${ }^{1,2}$ However, not all individuals with NCCAH are symptomatic and affected males are not usually detected until a female family member is diagnosed., ${ }^{1,2}$

Individuals with NCCAH may be compound het-

Key words: 17 -alpha-hydroxy-progesterone, Non-classical congenital adrenal hyperplasia

Address for correspondence:

Valeria Alcantara-Aragon, Endocrinology Department, Hospital de la Santa Creu I Sant Pau, Sant Antoni Maria Claret 167, Barcelona 08025, Spain, Tel.: +30 935565661, Fax: +30 935565602, e-mail: VAlcantara@santpau.cat Received 03-03-2013, Accepted 14-04-2014 erozygotes and can carry severe $C Y P 21 A 2$ allele mutations. ${ }^{2}$ Consequently, genetic testing and counseling is highly recommended when planning pregnancy. Our aim was to assess CYP21A2 allele mutations and review the clinical characteristics in patients with a NCCAH diagnosis attended at the center, irrespective of their need for genetic counseling at short term.

Patients with a diagnosis of NCCAH were recruited when they came for their regular follow-up visits. Medical records were examined to retrieve data about initial clinical presentation, later signs and symptoms, hormonal work-up [basal and stimulated 17-hydroxyprogesterone (17OHP)], and treatment at diagnosis and follow-up. A standardized database was used. The interpretation of the patients' hormonal work-up was performed according to Speiser PW et al: basal $17 \mathrm{OHP} \geq 6$ and $<30 \mathrm{nmol} / \mathrm{L}$ was considered abnormal, while basal or stimulated $17 \mathrm{OHP} \geq 30$ and $<300 \mathrm{nmol} / \mathrm{l}$ was considered indicative of NCCAH. ${ }^{3}$ Analysis of the CYP21A2 gene was performed through polymerase chain reaction, sequencing, and family genetic testing when possible. ${ }^{4}$ Data obtained from descriptive statistic analysis is expressed as percentages and medians (interquartile range). Chi-square and Mann-Whitney tests were used for comparisons among groups. The Institutional Review Board gave special approval for the study. 
Results are summarized in Table 1. The audit included 29 women followed at the Pediatrics $(\mathrm{N}=5)$, Gynecology \& Obstetrics $(\mathrm{N}=6)$ or Endocrinology $\&$ Nutrition $(\mathrm{N}=18)$ Departments at our center. The initial clinical diagnosis was made in all of these departments, as well as in the Endocrinology Departments of other centers from 1976 to 2010. Twenty-four patients $(82.7 \%)$ were index cases. Age at first symptom was $15.8 \pm 8$ years and the median number of symptoms was 2 (1 to 3 ). The most common manifestations were hirsutism (69\%) and oligomenorrhea (48.3\%). Basal $17 \mathrm{OHP}$ at diagnosis was available in the clini-

Table 1. Summary of clinical information of women followed with a diagnosis of NCCAH

\begin{tabular}{lcc}
\hline Patient characteristics: & N or mean & \% or IQR \\
\hline Index case & 23 & 79.3 \\
Age at first symptom (years, mean + SD) & 15.8 & 8 \\
Number of symptoms, median (IQR) & 2 & 1 to 3 \\
\hline Most common manifestations: & $\mathbf{N}$ & $\mathbf{\%}$ \\
\hline Hirsutism & $\mathbf{2 0}$ & 69 \\
Oligomenorrhea & 14 & 48.3 \\
Infertility & $\mathbf{4}$ & 13.8 \\
Accelerated linear growth & $\mathbf{4}$ & 13.8 \\
Acne & $\mathbf{3}$ & 10.3 \\
Alopecia & $\mathbf{3}$ & 10.3 \\
Precocious puberty & $\mathbf{3}$ & 10.3 \\
\hline
\end{tabular}

\begin{tabular}{|c|c|c|}
\hline Hormonal work-up at diagnosis: & Availability, n (\%) & Concentration (nmol/l), median (IQR) \\
\hline Basal 17 OH-P & $22(75.9)$ & $14(5.75$ to 41$)$ \\
\hline ACTH stimulated 17 OH-P & $8(27.6)$ & $142(38.5$ to 177.75$)$ \\
\hline Pharmacological treatment \# & $\mathbf{N}$ & $\%$ \\
\hline None & 38 & 12.1 \\
\hline Corticosteroids: & 233 & 74.2 \\
\hline Dexamethasone & 188 & 59.8 \\
\hline Hydrocortisone & 44 & 14 \\
\hline Prednisone & 1 & 0.3 \\
\hline Other treatments, alone or a combination: & 43 & 13.7 \\
\hline Oral contraceptives & 27 & 8.5 \\
\hline Spironolactone & 15 & 4.8 \\
\hline Flutamide & 1 & 0.31 \\
\hline Genetic analysis: & $\mathbf{N}$ & $\%$ \\
\hline No mutations & 3 & 10.3 \\
\hline Heterozygous, mild mutation & 8 & 27.6 \\
\hline Homozygous, mild mutation & 9 & 31 \\
\hline Compound heterozygous, mild/severe & 9 & 31 \\
\hline Most frequent mutations: & $\mathbf{N} \ddagger$ & $\%$ \\
\hline Mild exon 7, Val281Leu & 28 & 62 \\
\hline Mild exon 10, Pro453Ser & 6 & 11.1 \\
\hline Deletion & 5 & 8.8 \\
\hline Severe exon 10, R483P & 3 & 6.6 \\
\hline Severe IVS2, 290-13 C>G & 2 & 4.4 \\
\hline Severe exon 7, His282Tyr & 1 & 2.2 \\
\hline
\end{tabular}

IQR: Interquartile range; \# in 314 follow-up visits; $\ddagger$ One patient had 3 mutations. 
cal records of 22 patients (75.9\%) and was $14 \mathrm{nmol} / \mathrm{l}$ (5.75 to 41). Stimulated 17OHP was available in 8 patients (27.6\%) and was $142 \mathrm{nmol} / \mathrm{l}$ (38.5 to 177.75$)$. In a substantial subset of patients in follow-up for NCCAH $(16,55.2 \%)$, hormonal work-up available in the clinical records was not sufficient to establish a NCCAH diagnosis.

Genetic analysis is summarized in Table 1. Genetic testing of parents or siblings was performed in 10 patients $(34.5 \%)$ to clarify the genetic diagnosis. The most common mutation ( 28 out of $45,62 \%$ ) was one that is known to be mild: Val281Leu. Globally, $37.9 \%$ of the genetic results did not confirm the alleged diagnosis. Other genes were not analyzed, but since $95 \%$ of cases are due to a mutation of the CYP21A2 gene, ${ }^{3}$ it is unlikely that more than one third of the women had a mutation in a different gene.

The rate of non-confirmatory genetic analysis was not significantly different in women with a satisfactory biochemical diagnosis of NCCAH (33.1\%) vs those without (50\%). Department of origin, the patient's index condition, age at initial presentation, and number of signs/symptoms did not differ between women with and without a genetic diagnosis of NCCAH (data not shown). Twenty-six women $(89.7 \%)$ received drug treatment for NCCAH at some point during follow-up, most frequently corticosteroids (23 women, $79.3 \%$ ). Similarly, at 314 follow-up visits, $87.9 \%$ of the women were receiving drug treatment, most frequently corticosteroids (74.2\%). Use of drug treatment did not differ in women with and without a genetic diagnosis of NCCAH ( 88.9 vs $90.9 \%$ at some point during follow-up, ns).

The main finding of this investigation is that a clinically significant subset of women followed and treated according to a diagnosis of NCCAH did not have a confirmatory genetic diagnosis. Some patients had been referred from other centers and access to lab tests at diagnosis and follow-up was limited. This could partly explain the less than optimal rate of available hormonal work-up at diagnosis. The limited sample size may have contributed to the non-significant differences in the characteristics of women with and without a genetic diagnosis of NCCAH.
All women included in this series presented symptoms suggestive of NCCAH together with at least an abnormal basal 17OHP at some point that could help understand the assignment of a NCCAH diagnosis. The heterozygote condition was not uncommon. In the literature, the clinical presentation and $17 \mathrm{OHP}$ values of the heterozygote carriers may overlap with that of NCCAH. ${ }^{2}$ Other conditions, such as premature adrenarche in childhood or PCOS in young adults, might have been present. For example, Pall et $\mathrm{al}^{5}$ reported an elevation of basal $17 \mathrm{OHP}$ in up to $25 \%$ of patients with PCOS.

Current guidelines emphasize limiting drug treatment to symptomatic NCCAH patients and avoiding corticosteroids whenever possible, with the exception of women seeking pregnancy. ${ }^{3}$ At present we can only speculate on the potential untoward effects of prolonged treatment for a non-existing NCCAH.

The results of this audit compel us to reconsider the diagnostic and therapeutic requirements of patients coming to the outpatient clinic with an alleged diagnosis of NCCAH. The fact that a significant percentage of patients attended in different departments with a NCCAH diagnosis did not have a confirmatory genetic analysis suggests that the situation may not be uncommon.

\section{REFERENCES}

1. White PC, Speiser PW, 2000 Congenital adrenal hyperplasia due to 21-hydroxylased Deficiency. Endocr Rev 21: 245-291.

2. Witchel SF, 2013 Non-classic congenital adrenal hyperplasia. Steroids 78: 747-750.

3. Speiser PW, Azziz R, Baskin LS, et al, 2010 Congenital Adrenal Hyperplasia Due to Steroid 21-Hyroxylase Deficiency: An Endocrine Society Clinical Practice Guideline. J Clin Endocrinol Metab 95: 4133-4160.

4. Bidet M, Bellanné-Chantelot C, Galand-Portier MB, et al, 2009 Clinical and molecular characterization of a cohort of 161 unrelated women with nonclassical congenital adrenal hyperplasia due to 21-hydroxylase deficiency and 330 family members. J Clin Endocrinol Metab 94: 1570-1578.

5. Pall M, Azziz R, Beires J, et al, 2010 The phenotype of hirsute women: a comparison of polycystic ovary syndrome and 21-hydroxylase-deficient nonclassic adrenal hyperplasia. Fertil Steril 94: 684-689. 


\title{
The metabolic syndrome among preschool and school age children and adolescents in Crete in the first decade of the $21^{\text {st }}$ century
}

\author{
Christos M. Hatzis, ${ }^{1}$ Christopher Papandreou, ${ }^{2}$ Dimitra Sifaki-Pistolla,${ }^{3}$ \\ Christine Jildeh, ${ }^{3}$ Anthony G. Kafatos ${ }^{1}$
}

${ }^{1}$ Department of Social Medicine, Preventive Medicine and Nutrition Clinic, Medical School, University of Crete, Heraklion; ${ }^{2}$ Department of Nutrition \& Dietetics, Harokopio University of Athens, Athens; ${ }^{3}$ Department of Social Medicine, Clinic of Social and Family Medicine, University of Crete, Heraklion; Greece

\section{Dear Sir,}

In Greece as well as worldwide, studies on the prevalence of metabolic syndrome (MetS) in children/ adolescents began to take place in the second half of the $20^{\text {th }}$ century. It was indicated that the prevalence of MetS is mostly predicted by the presence of obesity and particularly abdominal obesity. ${ }^{1}$ A previous Greek study showed that the prevalence of MetS was higher in overweight/obese children compared to normal weight controls. ${ }^{2}$ Recently, a Greek National Epidemiological Survey found a prevalence of high abdominal obesity among children 6-12 years old, higher than that recently reported from other European countries. ${ }^{3}$ Crete, a Greek island known for its favorable health status in the 1960s, experienced a rise in the prevalence of childhood obesity between 1982 and 2002, ${ }^{4}$ assumed to also be accompanied by increases in MetS prevalence. To our knowledge, only one previous study has examined the prevalence of MetS among Cretan children, this carried out during the period 2001-2003. ${ }^{5}$ Such studies are important

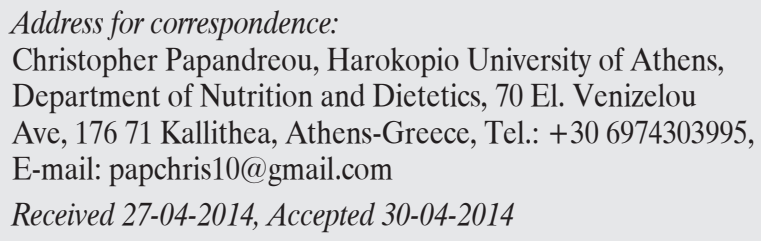

from a public health perspective, since identification of MetS factors that predispose to morbidity could help public health professionals to develop more effective preventive measures. Therefore, this study aims to determine the prevalence of MetS in a school-based sample of school children and adolescents in Crete extending the above period to 2011.

This study used secondary data from overweight and obesity registries in kindergarten, elementary and junior high school children during the years 20012011, from different regions of Chania and Heraklion prefectures in Crete. The selected children, presented in Table 1, participated in the Clinical Preventive Medicine and Nutrition program of the School of Medicine at the University of Crete. Initially, 2968 children/adolescents were recruited, of whom 1088 agreed to undergo anthropometric and biochemical measurements (blood tests). Of the participants, 887 were boys and 993 were girls aged 3-19 years.

Waist circumference (WC) was measured to the nearest $0.1 \mathrm{~cm}$ with the use of a non-elastic tape, with the pupil standing, at the end of a gentle expiration after placing the measuring tape on a horizontal plane around the trunk, at the level of umbilicus midway, between the lower rib margin and the iliac crest. The age- and sex-specific WC percentiles were used for the classification of central obesity ( $\geq 90$ th percentile). 
Table 1. Descriptive statistics of the study population

\begin{tabular}{|c|c|c|c|c|c|c|c|}
\hline $\mathbf{A} / \mathbf{A}$ & Study groups & $\mathbf{N}(\%)^{*}$ & $\begin{array}{c}\text { Metabolic } \\
\text { Syndrome } \\
\text { Components }\end{array}$ & Boys & Girls & $\begin{array}{c}\text { Age } \\
\text { (years) }\end{array}$ & $\begin{array}{c}\text { Year of } \\
\text { assessment }\end{array}$ \\
\hline 1 & Administrative region of Rouvas (students) & $192(94)$ & 188 & 89 & 99 & $4-16$ & 2001 \\
\hline 2 & Administrative region of Chania (students) & $303(68)$ & 246 & 103 & 143 & $14-17$ & 2002 \\
\hline 3 & $\begin{array}{l}\text { Administrative region of Chersonissos } \\
\text { (preschool children) }\end{array}$ & $110(63)$ & 89 & 46 & 43 & $3-7$ & 2003 \\
\hline 4 & $\begin{array}{l}\text { Administrative region of Heraklion (Dilina, } \\
\text { Tilissos - preschool children and students) }\end{array}$ & 779 (67) & 686 & 304 & 382 & $3-17.5$ & 2003 \\
\hline 5 & $\begin{array}{l}\text { Six administrative areas of the county } \\
\text { of Chania (preschool children) }\end{array}$ & $1356(77)$ & 574 & 297 & 277 & $4-7$ & 2004 \\
\hline 6 & $\begin{array}{l}\text { Administrative region of Minoa-Pediados } \\
\text { (students) }\end{array}$ & $228(74)$ & 97 & 48 & 49 & $12-19$ & 2011 \\
\hline Total & & 2968 & 1880 & 887 & 993 & & 2001-2011 \\
\hline
\end{tabular}

*Children who participated in each study $(\mathrm{N}=$ total number and $\%=$ percentage $)$.

Blood pressure was measured in the right arm while in a sitting position and after five minutes of rest. A mercury sphygmomanometer was used covering from 50 to $75 \%$ of the perimeter of the right arm. The measurement was taken twice with a two-minute interval between readings. A third measurement was taken if there was a difference of over $10 \mathrm{mmHg}$ between the previous measurements. The average value of the measurements was used in analysis. Systolic (SBP) and diastolic blood pressure (DBP) were recorded. Systolic or diastolic hypertension was defined as SBP or DBP above the 95th percentile for gender, age and height. ${ }^{6}$

Early morning blood samples were taken after a 12-hour overnight fast. The parents as well as the children were reminded on the previous day in order to ensure compliance with fasting. Plasma glucose, total cholesterol, triglycerides, high density lipoprotein cholesterol (HDL-C) and low density lipoprotein cholesterol were measured (methods referenced in Hatzis et al). ${ }^{7}$ The National Cholesterol Education Program cut-off points for blood lipids were used to define dyslipidemias. ${ }^{8}$

The MetS was defined as the presence of $\geq 3$ of the following factors among children/adolescents: impaired fasting blood glucose, hypertension, abdominal obesity, hypertriglyceridemia and low HDL-C.

The statistical analysis was conducted in SPSS
20. Descriptive statistics were exported for all variables and are presented in the accompanying tables. No extended statistical tests are presented in order not to overestimate the results that were treated as secondary data.

Table 2 shows the frequencies of MetS among children and adolescents in Crete (2001-2011). More

Table 2. Metabolic syndrome components of children and adolescents in Crete (2001-2011)

\begin{tabular}{lccc}
\hline Factors & Boys N (\%) & Girls N (\%) & Total N (\%) \\
\hline 0 & $472(53.2)$ & $505(50.8)$ & $977(52.0)$ \\
1 & $287(32.4)$ & $364(36.7)$ & $651(34.6)$ \\
2 & $95(10.7)$ & $100(10.1)$ & $195(10.4)$ \\
$>=3$ (MetS) & $33(3.7)$ & $24(2.4)$ & $57(3.0)$ \\
Total & 887 & 993 & 1880 \\
\hline
\end{tabular}

$\mathrm{N}=$ total number; $\%=$ percentage.

Metabolic syndrome components are described as follows:

1) Hyperglycemia: blood sugar serum $\geq 100 \mathrm{mg} / \mathrm{l}$

2) Hypertension: systolic blood pressure $>95^{\text {th }}$ percentile (genderage) or diastolic blood pressure $>95^{\text {th }}$ percentile (gender-age) and 16.5 years $\geq 130 / 85 \mathrm{mmHg}$

3) Central obesity: waist circumference $>90^{\text {th }}$ percentile and $>16.5$ years $\geq 94 \mathrm{~cm}$ and $80 \mathrm{~cm}$ for boys and girls, respectively

4) Hypertriglyceridemia: triglycerides $\geq 100 \mathrm{mg} / \mathrm{dl}$ and $>16.5$ years $\geq 150 \mathrm{mg} / \mathrm{dl}$

5) HDL-cholesterol: low $<10^{\text {th }}$ percentile (gender-age) and $>16.5$ years $<40 \mathrm{mg} / \mathrm{dl}$

* For boys and girls aged $\geq 16.5$ years, the criteria for adult men and women were used, respectively. 
than half of the children and adolescents were found to have no MetS risk factor (52\%: $53.2 \%$ boys; $50.8 \%$ girls), $34.6 \%$ of the children/adolescents were found to have only one risk factor ( $32.4 \%$ boys; $36.7 \%$ girls), while $10.4 \%$ were found to have two risk factors for MetS (10.7\% boys; $10.1 \%$ girls). Of the total sample, $48 \%$ had $>1$ risk factors for MetS $(46.8 \%$ boys versus $49.2 \%$ girls). Finally, $3 \%$ of the children/adolescents were identified as having three or more risk factors for MetS (3.7\% boys; $2.4 \%$ girls).

In a previous study among elementary school children of north-east Attica, the prevalence of MetS was estimated at 3.6\%. ${ }^{9}$ From worldwide surveys on the prevalence of MetS in children and adolescents, it is estimated that the prevalence of Mets is around $4-5 \%,{ }^{10,11}$ and this prevalence increases with obesity. Cook S. et al ${ }^{12}$ found that $6.8 \%$ of overweight and $28.7 \%$ of obese children and adolescents had MetS, while Duncan et $\mathrm{al}^{13}$ found higher rates of Mets among overweight (7.1\%) and obese $(32.1 \%)$ children/adolescents. As concerns children aged 2 to 20 years, a previous study found that in those with a BMI over the 95th percentile, the prevalence of MetS reached $40 \% .{ }^{14}$ Another study conducted by Cruz et $\mathrm{al}^{15}$ showed that the prevalence of Mets syndrome was $6.8 \%$ among overweight children, while it was $28.7 \%$ among obese children.

The presence of Mets in childhood is crucial because there is evidence that the syndrome in childhood could predict cardiovascular disease in adulthood. Among children, and especially among those with MetS risk factors, adoption of the traditional Mediterranean diet, which is rich in antioxidant and anti-inflammatory substances, engaging in regular exercise and participation in long-term educational intervention programs is the only way to prevent and reduce those factors that affect and continuously increase morbidity and mortality from chronic diseases.

\section{REFERENCES}

1. Alberti G, Zimmet PZ, Shaw JE, 2005 IDF Epidemiology Task Force Consensus Group. The metabolic syndrome - a new word-wide definition from the International Diabetes Federation Consensus. Lancet 366: 1059-1062.
2. Filippou O, Karanasiou K, Dolianiti M, et al, 2008 Obesity and the metabolic syndrome in greek children and adolescents. Arch Dis Child 93: eap10.

3. Tzotzas T, Kapantais E, Tziomalos K, et al, 2011 Prevalence of overweight and abdominal obesity in Greek children 6-12 years old: Results from the National Epidemiological Survey. Hippokratia 15: 48-53.

4. Manios Y, Magkos F, Christakis G, et al, 2005 Twentyyear dynamics in adiposity and blood lipids of Greek children: regional differences in Crete persist. Acta Paediatr 94: 859-865.

5. Linardakis M, Sarri K, Bertsias G, Kafatos A, 2008 Division of Preventive Medicine and Nutrition. Metabolic syndrome in children and adolescents in Crete, Greece, and association with diet quality and physical fitness. J Public Health 16: 421-428.

6. National High Blood Pressure Education Program Working Group on High Blood Pressure in Children and Adolescents, 2004 The fourth report on the diagnosis, evaluation, and treatment of high blood pressure in children and adolescents. Pediatrics 114: 555-576.

7. Hatzis CM, Papandreou C, Vardavas C, Athanasopoulos D, Balomenaki E, Kafatos AG, 2012 Atherogenic risk factors among preschool children in Crete,Greece. Indian J Endocrinol Metab 16: 809-814.

8. National Heart Lung and Blood Institute, 2011 Expert panel on integrated guidelines for cardiovascular health and risk reduction in children and adolescents: summary report. Pediatrics 128: Supp 15: 213-256.

9. Christodoulos A, Tokmakidis S, Douda E, Tosoulis D, Gikas E, 2008 Documented and Newer Cardiovascular Risk Factors in Childhood. Arch Hellen Med 25: 1-9.

10. Molnar D, 2004 The prevalence of the metabolic syndrome and type 2 diabetes mellitus in children and adolescents. Int J Obes Relat Metab Disord 28: Suppl 3: 70-74.

11. Jessup A, Harell JS, 2005 The metabolic syndrome: look for it in children and adolescents, too! Clin Diabetes 23: $26-32$.

12. Cook S, Weitzman M, Auinger P, Nguyen M, Dietz WH, 2003 Prevalence of metabolic syndrome phenotype in adolescents: findings from the third National Health and Nutrition Health and Nutrition Examination Survey, 1988-1994. Arch Pediatr Adolesc Med 157: 821-827.

13. Duncan GE, Li SM, Zhou XH, 2004 Prevalence and trends of a metabolic syndrome phenotype among U.S. Adolescents 1999 -2000. Diabetes Care 27: 2438 -2443.

14. Weiss R, Dziura J, Burgert TS, 2004 Obesity and the Metabolic Syndrome in children and adolescents. N Eng J Med 350: 2362-2374.

15. Cruz ML, Goran MI, 2004 The metabolic syndrome in children and adolescents. Curr Diab Rep 4: 53-62. 


\section{Order Form - Subscription}

Please enter my subscription to HORMONES

$$
\square \text { Print Subscription }
$$

$\square$ I am a member of

$\square$ Please despatch my issues by airmail

$\square$ My VAT registration number is .................. $\square \mathrm{I}$ am not VAT-registered

$\square$ I attach a cheque (payable to Hellenic Endocrine Society ) for the sum of ...60 Euro

$\square$ Please debit my American Express/Diners/Eurocard//CB/Mastercard/VISA/Switch

Card no:

$\square$

Issue no:
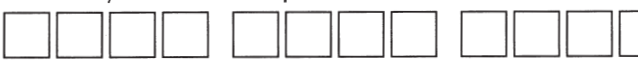

Signature

with the sum of

$\square$ Please send me an invoice

Name

Address

Street/PO Box

County

Town

Country

Post/Zip Code

Telephone No.

E-mail 
HORMONES publishes articles related to:

* Original research findings on humans or experimental animals

* Translational research

* Complete reviews of the Literature

* Case reports will exceptionally be considered only when they present some novel mechanism or previously unknown mutation etc

* Editorial Commentaries

* News and Views

* Book Reviews

* Letters to the Editor

* Short notes on etymology of medical terms

* History of Endocrinology

PREPARATION OF MANUSCRIPTS

* Must be written in correct English.

* Use wide margins

* Number all pages

* Use separate pages for title page, references, footnotes, tables, legends

* Figures should be created with high resolution software

The title page should include:

* Full title

* Abbreviated title of not more than 40 characters

* Authors' names and institution affiliations

* Corresponding author's address, telephone number, fax and e-mail

* Key words up to 8

* Grants or fellowship supports

* Any conflict of interest

\section{ABSTRACT}

For research papers the abstract must be structured: OBJECTIVE, DESIGN, RESULTS, CONCLUSIONS. It should not exceed 250 words. An abstract is not necessary for review articles.

Describe briefly the background, the aim of the study or the hypothesis tested, the methods used, the results and the conclusions.

\section{MAIN TEXT}

Follow the usual architectural model, namely: introduction, subjects or experimental animals and methodology, results, discussion.

Laboratory values should be stated in both the international system (SI units) and in metric units in parenthesis. Values in the abstract values should be given only in SI units. Temperatures should be written in degrees Celsius.

\section{FOOTNOTES}

Footnotes should be avoided. When considered necessary they should be numbered consecutively and placed at the foot of the appropriate page.

\section{FIGURES}

For electronic submission use a separate file for each figure in one of the three acceptable formats TIF, EPS, JPG (at least 300dpi). Figure legends should contain all the information necessary without reference to the text. Number in Arabic numericals. TABLES should be presented on separate pages as printed text. Tables require a concise heading.

\section{REFERENCES}

References should be cited consecutively in numerical order in the text (as superscript outside the punctuation) and the same numerical order must be followed on a separate sheet at the end of the manuscript. The title of the Journals used follow the abbreviated style used in the index medicus.

The author is responsible for the accuracy of references.

\section{Examples}

- Papers published in Journals:

Mahagan T, Lightman SL, 2000 A simple test for growth hormone deficiency in adults. J Clin Endocrinol Metab 85: 1473-1476.

When the number of authors exceeds six, the designation et al must be used after the third author.

\section{- Books:}

Mazzaferri EL 1993 Thyroid carcinoma. Papillary and follicular. In: Mazzaferri EL, Samaan N (eds) Endocrine tumors, Blackwell Scientific Publications Inc, Cambridge; pp, 278-333.

\section{SUBMISSION PROCEDURE \\ - Electronic}

Only electronic submission is now possible. Please submit through our website www.hormones.gr

- Create an account as an author

- Log in to our system as an author

- Follow the on-line instructions step by step

- The home page also includes FAQs for any further information you may need.

www.hormones-submissions.gr/index.php/system/login

- Provide a statement signed by all authors that the manuscript has not been published or submitted elsewhere.

State the first and the last name of all the authors, their e-mail address and the institution they work for.

Provide the full name of at least four possible reviewers experts in the relevant field of research for the submitted article together with their e-mail address.

- Personal communications should not be included in the references. They may be cited in the text in parentheses. Papers in press may be mentioned in the references given in the Journal. Manuscripts will become the permanent property of HORMONES and should not be published elsewhere without written permission from the authors and Journal.

Manuscripts are submitted to HORMONES with the understanding that they have not been simultaneously submitted to other journals

\section{ETHICAL CONSIDERATIONS}

Pulished research must comply with the guidelines for human studies and animal welfare regulations. Authors should state these, when applicable. The relevant statement should be accompanied by a notation of approval by the institutional committee on human research in the Materials and Methods section.

\section{CONFLICT OF INTEREST}

Authors should disclose any sponsorship or funding related to their research. All authors should disclose any conflicts of interest.

\section{PLAGIARISM}

This is defined as the case where a paper includes repetition of another text published elsewhere with a similarity of at least $25 \%$ without citation. If such plagiarism is identified either before or after publication authors will be asked for explanations and there is risk that the paper may be retracted. 